\title{
Double-scale Gevrey asymptotics for logarithmic type solutions to singularly perturbed linear initial value problems
}

\author{
S. Malek \\ University of Lille, Laboratoire Paul Painlevé, \\ 59655 Villeneuve d'Ascq cedex, France, \\ Stephane.Malek@univ-lille.fr
}

\begin{abstract}
We examine a family of linear partial differential equations both singularly perturbed in a complex parameter $\epsilon$ and singular in complex time $t$ at the origin. These equations entail forcing terms which combine polynomial and logarithmic type functions in time and that are bounded holomorphic on horizontal strips in one complex space variable. A set of sectorial holomorphic solutions are built up by means of complete and truncated Laplace transforms w.r.t $t$ and $\epsilon$ and Fourier inverse integral in space. Asymptotic expansions of these solutions relatively to $t$ and $\epsilon$ are investigated and two distinguished Gevrey type expansions in monomial and logarithmic scales are exhibited.

Key words: asymptotic expansion, Borel-Laplace transform, Fourier transform, initial value problem, formal power series, singular perturbation. $2000 \mathrm{MSC}$ : 35C10, 35C20.
\end{abstract}

\section{Introduction}

In this paper, we focus our attention on a family of singularly perturbed linear partial differential equations with the shape

$$
Q\left(\partial_{z}\right) u(t, z, \epsilon)=(\epsilon t)^{d_{D}}\left(t \partial_{t}\right)^{\delta_{D}} R_{D}\left(\partial_{z}\right) u(t, z, \epsilon)+P\left(t, z, \epsilon, t \partial_{t}, \partial_{z}\right) u(t, z, \epsilon)+f(t, z, \epsilon)
$$

for vanishing initial data $u(0, z, \epsilon) \equiv 0$, where $d_{D}, \delta_{D} \geq 1$ are integers, $Q(X), R_{D}(X)$ stand for polynomials with complex coefficients and $P\left(t, z, \epsilon, V_{1}, V_{2}\right)$ represents a polynomial in the arguments $t, V_{1}, V_{2}$ with holomorphic coefficients relatively to the perturbation parameter $\epsilon$ on a disc $D_{\epsilon_{0}}$ with radius $\epsilon_{0}>0$ centered at 0 and holomorphic w.r.t the space variable $z$ on a horizontal strip in $\mathbb{C}$ framed as $H_{\beta}=\{z \in \mathbb{C} /|\operatorname{Im}(z)|<\beta\}$, for some given width $2 \beta>0$.

The forcing term $f(t, z, \epsilon)$ involves coefficients that depend polynomially on the time variable $t$, analytically on $\epsilon$ on $D_{\epsilon_{0}}$ and holomorphically in $z$ on $H_{\beta}$. This term combines also logarithmic type functions expressed as truncated Laplace transforms along a fixed segment $[-a, 0]$ for some radius $a>0$ that count in the function $1 / \log (\epsilon t)$. Its expression is chosen in a way that when $a>0$ is taken large, it becomes proximate to a general logarithmic type map $f_{\infty}$ in $t, \epsilon$ displayed as polynomials in both $\epsilon t$ and $1 / \log (\epsilon t)$ with coefficients that are entire functions on the strip $H_{\beta}$, see (24). It is worth noticing that this radius $a>0$ can be taken as large as desired provided that the radius $\epsilon_{0}>0$ of $D_{\epsilon_{0}}$ is chosen close enough to the origin. However, $a>0$ cannot be set 
to be infinite $(a \neq+\infty)$. The explicit constraints relating these two quantities are given through the technical bounds (79) and (89).

Observe that the main equation (1) involves powers of the basic differential operator of Fuchsian type $t \partial_{t}$. For a conspicuous textbook about Fuchsian ordinary and partial differential equations, we refer to [7]. However, under the sufficient conditions set on (1) listed in Subsection 2.2 , it turns out that (1) will be reduced throughout the work to an auxiliary prominent equation, stated in (41), that brings in only powers of basic differential operators of so-called irregular type $u_{1}^{k_{1}+1} \partial_{u_{1}}$ and $u_{2}^{2} \partial_{u_{2}}$ in two independent complex variables $u_{1}$ and $u_{2}$. The definition of irregular type differential operators can be found in the classical textbook [1] in the framework of ordinary differential equations and in the work [15] in the context of partial differential equations.

Remark that the limit map $f_{\infty}$ displayed in (24) (obtained from the forcing term as its truncated Laplace radius $a$ tends to $+\infty$ ) is made up with pieces that separately solve explicit nonlinear ordinary differential equations that are singularly perturbed and comprise first order differential operators of irregular type, see (25).

In the present study, our objective is the construction of a set of holomorphic solutions to (1) and the description of their asymptotic expansions as $\epsilon$ tends to 0 (stated in Theorem 1 of Subsection 4.3). We model these solutions as functions representable as double Laplace transforms and Fourier integral. Such an approach has already been successfully applied in the recent works [11], [12] by A. Lastra and the author and in [3] by G. Chen, A. Lastra and the author in the analysis of singularly perturbed initial value problems in two complex time variables. Under the list of conditions applied to the shape of (1), detailed in Subsection 2.2, one can single out

- A set of suitably chosen bounded open sectors $\left\{\mathcal{E}_{p}\right\}_{p \in I_{1}}$ for some finite set $I_{1} \subset \mathbb{N}$ and $\mathcal{T}$ centered at 0 .

- Appropriate directions $d_{p} \in \mathbb{R}, p \in I_{1}$, for which a family of holomorphic solutions $u_{p}(t, z, \epsilon)$ to (1) can be built up on the domains $\mathcal{T} \times H_{\beta} \times \mathcal{E}_{p}$. Each solution $u_{p}, p \in I_{1}$, is expressed as a complete Laplace transform of some integer order $k_{1} \geq 1$ in the monomial $\epsilon t$, a truncated Laplace transform of order 1 in the logarithmic map $1 / \log (\epsilon t)$ and an inverse Fourier integral in the space variable $z$,

$$
\begin{aligned}
u_{p}(t, z, \epsilon)=\frac{k_{1}}{(2 \pi)^{1 / 2}} \int_{L_{d_{p}}} \int_{[-a, 0]} \int_{-\infty}^{+\infty} \omega_{p} & \left(\tau_{1}, \tau_{2}, m, \epsilon\right) \\
& \times \exp \left(-\left(\frac{\tau_{1}}{\epsilon t}\right)^{k_{1}}-\left(\log (\epsilon t) \tau_{2}\right)\right) e^{i z m} \frac{d \tau_{1}}{\tau_{1}} \frac{d \tau_{2}}{\tau_{2}} d m
\end{aligned}
$$

where the so-called Borel/Fourier map $\omega_{p}\left(\tau_{1}, \tau_{2}, m, \epsilon\right)$ stands for a function

- which is analytic near $\tau_{1}=0$ and for $\tau_{2} \in D_{a}$,

- with (at most) of exponential growth of order $k_{1}$ on an infinite sector containing the half line $L_{d_{p}}=[0,+\infty) e^{\sqrt{-1} d_{p}}$ w.r.t $\tau_{1}$,

- continuous and under exponential decay w.r.t $m$ on $\mathbb{R}$,

- with analytic reliance on $\epsilon$ in the punctured disc $D_{\epsilon_{0}} \backslash\{0\}$.

According to the very structure of these solutions, the family $\left\{u_{p}\right\}_{p \in I_{1}}$ owns asymptotic expansions of Gevrey type in two particular scales of functions. 
All the functions $\epsilon \mapsto u_{p}(t, z, \epsilon), p \in I_{1}$, share a common asymptotic formal expansion

$$
\hat{u}^{1}(t, z, \epsilon)=\sum_{n \geq 0} G_{n}^{1}(t, z, \epsilon) \frac{\epsilon^{n}}{n !}
$$

on $\mathcal{E}_{p}$, in the scale of monomials $\left\{\epsilon^{n}\right\}_{n \geq 0}$, with bounded holomorphic coefficients $G_{n}^{1}$ on $\mathcal{T} \times H_{\beta} \times$ $\mathcal{D}_{\epsilon_{0}}$, where $\mathcal{D}_{\epsilon_{0}}$ is an open domain of $D_{\epsilon_{0}} \backslash\{0\}$ given by (134). Furthermore, these asymptotic expansions turn out to be of Gevrey order $1 / k_{1}$ on every sectors $\mathcal{E}_{p}$, meaning that constants $K_{p}^{1}, M_{p}^{1}>0$ can be chosen for which the error bounds

$$
\left|u_{p}(t, z, \epsilon)-\sum_{n=0}^{N} G_{n}^{1}(t, z, \epsilon) \frac{\epsilon^{n}}{n !}\right| \leq K_{p}^{1}\left(M_{p}^{1}\right)^{N+1} \Gamma\left(1+\frac{N+1}{k_{1}}\right)|\epsilon|^{N+1}
$$

hold for all integers $N \geq 0$, all $\epsilon \in \mathcal{E}_{p}$, uniformly in $t \in \mathcal{T}$ and $z \in H_{\beta}$. In Proposition 9, we show that the coefficients $G_{n}^{1}$ are subjected to an explicit differential recursion relation w.r.t $n \geq 0$ that may be useful for their effective computations.

For each $p \in I_{1}$, the function $(t, \epsilon) \mapsto u_{p}(t, z, \epsilon)$ possesses a generalized asymptotic formal expansion (in the sense defined in the classical textbooks [6], [18])

$$
\hat{u}_{p}^{2}(t, z, \epsilon)=\sum_{n \geq 0} G_{n, p}^{2}(t, z, \epsilon) \frac{(1 / \log (\epsilon t))^{n}}{n !}
$$

on the domain $\mathcal{T} \times \mathcal{E}_{p}$, in the scale of logarithmic functions $\left\{(1 / \log (\epsilon t))^{n}\right\}_{n \geq 0}$, for bounded holomorphic coefficients $G_{n, p}^{2}$ on $\mathcal{T} \times H_{\beta} \times \mathcal{E}_{p}$. These asymptotic expansions share the common feature to be of Gevrey order 1 on the sectors $\mathcal{E}_{p}$, giving rise to constants $K^{2}, M^{2}>0$ for which the error estimates

$$
\left|u_{p}(t, z, \epsilon)-\sum_{n=0}^{N} G_{n, p}^{2}(t, z, \epsilon) \frac{(1 / \log (\epsilon t))^{n}}{n !}\right| \leq K^{2}\left(M^{2}\right)^{N+1} \Gamma(N+2)|1 / \log (\epsilon t)|^{N+1}
$$

occur for all integers $N \geq 0$, all $\epsilon \in \mathcal{E}_{p}$ and $t \in \mathcal{T}$, uniformly in $z \in H_{\beta}$. In addition, the coefficients $G_{n, p}^{2}$ are proved to fulfill some partial differential recursion relation in regard to $n \geq 0$ that may be helpful for their practical reckoning, see Proposition 10 .

The functions $u_{p}, p \in I_{1}$, are expressed in terms of maps $\mathbb{U}_{d_{p}, \pi}\left(t, u_{2}, z, \epsilon\right)$ in four complex variables through

$$
u_{p}(t, z, \epsilon)=\mathbb{U}_{d_{p}, \pi}\left(t, \frac{1}{\log (\epsilon t)}, z, \epsilon\right)
$$

which turn out to be embedded into a larger family of maps $\mathbb{U}_{d_{p}, \mathrm{~d}_{q}}\left(t, u_{2}, z, \epsilon\right)$ for all integers $(p, q) \in\left\{0, \ldots, \varsigma_{1}-1\right\} \times\left\{0, \ldots, \varsigma_{2}-1\right\}$ for some integers $\varsigma_{1}, \varsigma_{2} \geq 2$. These maps are bounded holomorphic on products $\mathcal{T} \times U_{2, \mathfrak{o}_{q}} \times H_{\beta} \times \mathcal{E}_{p}$ where

- $\underline{\mathcal{U}}_{2}=\left\{U_{2, \mathfrak{d}_{q}}\right\}_{0 \leq q \leq \varsigma_{2}-1}$ is a set of bounded sectors with bisecting directions $\mathfrak{d}_{q} \in \mathbb{R}$ forming a good covering in $\mathbb{C}^{*}$ (see Definition 5) with $\mathfrak{d}_{q_{1}}=\pi$ for some integer $q_{1} \in\left\{0, \ldots, \varsigma_{2}-1\right\}$.

- $\underline{\mathcal{E}}=\left\{\mathcal{E}_{p}\right\}_{0 \leq p \leq \varsigma_{1}-1}$ stands for a set of bounded sectors, containing $\mathcal{E}_{p}$ for $p \in I_{1}$, representing a good covering in $\mathbb{C}^{*}$.

Each map $\mathbb{U}_{d_{p}, \mathfrak{o}_{q}}$ is modeled as a rescaled version of a bounded holomorphic map $\left(u_{1}, u_{2}, z\right) \mapsto$ $U_{d_{p}, \mathfrak{d}_{q}}\left(u_{1}, u_{2}, z, \epsilon\right)$ by means of

$$
\mathbb{U}_{d_{p}, \mathfrak{d}_{q}}\left(t, u_{2}, z, \epsilon\right)=U_{d_{p}, \mathfrak{d}_{q}}\left(\epsilon t, u_{2}, z, \epsilon\right)
$$


on domains $U_{1, d_{p}} \times U_{2, \mathfrak{o}_{q}} \times H_{\beta}$ for all $\epsilon \in D_{\epsilon_{0}} \backslash\{0\}$ where $U_{1, d_{p}}$ are bounded sectors bisected by the directions $d_{p}$, described in Definition 6 of the work. These maps $U_{d_{p}, \mathfrak{o}_{q}}\left(u_{1}, u_{2}, z, \epsilon\right)$ are shown to solve a set of auxiliary linear partial differential equations given by (104) which combine powers of the basic differential operators $u_{1}^{k_{1}+1} \partial_{u_{1}}$ and $u_{2}^{2} \partial_{u_{2}}$ as brought to light in (41). This implies in particular that the functions $u_{p}, p \in I_{1}$, solve our main problem (1) on $\mathcal{T} \times H_{\beta} \times \mathcal{E}_{p}$.

The two asymptotic properties (2) and (3) for $u_{p}$ stem from sharp exponential bound estimates for the differences of neighboring maps $\mathbb{U}_{d_{p}, \mathbb{d}_{q}}$ reached in Proposition 7 , for which a well known criterion for the existence of asymptotic expansions of Gevrey type established by J-P. Ramis and Y. Sibuya can be applied, see Subsection 4.2.

In the framework of linear partial differential equations of so-called Fuchsian type, the construction of logarithmic type solutions is a well established subject. In the papers [19], [20], H. Tahara considers so-called linear Fuchsian partial differential equations (introduced by M. Baouendi and C. Goulaouic in [2]) with the shape

$$
t^{m} \partial_{t}^{m} u(t, x)+\sum_{j=1}^{m} P_{j}\left(t, x, \partial_{x_{1}}, \ldots, \partial_{x_{n}}\right) t^{m-j} \partial_{t}^{m-j} u(t, x)=0
$$

for linear differential operators $P_{j}$, with order less than $j$, with holomorphic coefficients near $t=0$ and $x=\left(x_{1}, \ldots, x_{n}\right)=0$. Under conditions of non resonance of the characteristic exponents at $x=0$, he has characterized the holomorphic solutions to (4) on $\widetilde{D_{T} \backslash\{0\}} \times D_{R}$ for a prescribed disc $D_{T}\left(\right.$ resp. $\left.D_{R}\right)$ centered at $t=0$ (resp. $\left.x=0\right)$ with radius $T>0$ (resp. $R>0$ ) where $\widetilde{D_{T} \backslash\{0\}}$ stands for the universal covering of $D_{T} \backslash\{0\}$, which can be expressed as

$$
u(t, x)=\sum_{l=1}^{m} t^{\lambda_{l}(x)} \sum_{j=0}^{+\infty} t^{j} u_{l, j}(t, x)
$$

where $u_{l, j}(t, x)=\sum_{k=1}^{r_{l, j}} u_{l, j, k}(x)(\log (t))^{k-1}$, for positive integers $r_{l, j} \geq 1$ and holomorphic maps $u_{l, j, k}$ on $D_{R}$, where $\lambda_{l}(x)$ represent the characteristic exponents of the equation (4) at $x$. Later on in the year 2000, T. Mandai was able to extend this important result to the general situation without any assumption on the characteristic exponents by following a similar approach to the method of Frobenius for ordinary differential equations with regular singularity at a point, see $[16]$.

In the context of linear partial differential equations of so-called irregular type in which our present contribution falls, much less results are known and represents a promising trend for upcoming research. Nevertheless, in that direction, we can mention the striking paper [23] by H. Yamazawa published in 2017. Therein, the author examines linear partial differential equations of the form

$$
C_{l}\left(t \partial_{t}, x\right) u(t, x)=\sum_{j+|\alpha| \leq m} a_{j, \alpha}(t, x)\left(t \partial_{t}\right)^{j} \partial_{x_{1}}^{\alpha_{1}} \cdots \partial_{x_{n}}^{\alpha_{n}} u(t, x)+f(t, x)
$$

for $x=\left(x_{1}, \ldots, x_{n}\right) \in \mathbb{C}^{n}$, with holomorphic coefficients $a_{j, \alpha}$ and forcing term $f$ near $(t, x)=$ $(0,0) \in \mathbb{C}^{n+1}$, for some monic polynomial $\rho \mapsto C_{l}(\rho, x)$ with holomorphic coefficients near $x=0$ with degree $1 \leq l<m$. Under technical conditions set on a so-called Newton polygon related to (6), the author constructs formal solutions with the shape $\hat{u}(t, x)=\hat{u}_{1}(t, x)+\hat{u}_{2}(t, \log (t), x)$ with

$$
\hat{u}_{1}(t, x)=\sum_{i \geq 1} u_{i}(x) t^{i} \quad, \quad \hat{u}_{2}(t, \log (t), x)=\sum_{p=1}^{\nu} t^{\rho_{p}(x)} \sum_{\substack{i \geq 0, 0 \leq k \leq m i}} \varphi_{i, k, p}(x) t^{i}(\log (t))^{k}
$$


where $\rho_{p}(x)$ are the roots of $\rho \mapsto C_{l}(\rho, x)$ with positive real parts at $x=0$ and $u_{i}(x), \varphi_{i, k, p}(x)$ are holomorphic coefficients on some small disc $D_{R}, R>0$. The formal series in $t, \hat{u}_{1}(t, x)$ (resp. $\hat{u}_{2}(t, y, x)$ ) with holomorphic coefficients on the domain $D_{R}$ (resp. $D_{R / \tau} \times D_{R}$ for any fixed small parameter $\tau>0$ ) are divergent in general but are shown to be (multi)-summable in several levels of Gevrey orders on suitable sectors (in the sense defined in the book [1]).

At last, we quote two compelling recent works that are somehow related to the result of the present study.

In [21], H. Tahara investigates higher order analogs of nonlinear singular partial differential equations of first order with so-called Briot-Bouquet type (see the textbook [7] by R. Gérard and H. Tahara for the origin of this terminology). These equations are written in the form

$$
\left(t \partial_{t}\right)^{m} u(t, x)=F\left(t, x,\left\{\left(t \partial_{t}\right)^{j} \partial_{x_{1}}^{\alpha_{1}} \cdots \partial_{x_{n}}^{\alpha_{n}} u\right\}_{j+|\alpha| \leq m, j<m}\right)
$$

for $x=\left(x_{1}, \ldots, x_{n}\right) \in \mathbb{C}^{n}$, under some restrictions of the analytic map $F(t, x, Z)$ near the origin. The author studies spaces of solutions $u(t, x)$ to $(7)$, that are holomorphic on a product $S \times D_{R}$ for some sector $S$ centered at 0 and given disc $D_{R}$ with radius $R>0$, restricted to upper bounds of the form

$$
\sup _{|x|<R}|u(t, x)| \leq \frac{C}{|\log (t)|^{a}}
$$

for some constants $C, a>0$ provided that $t \in S$. Sufficient conditions on the characteristic exponents at $x=0$ of (7) are given for which the bounds (8) imply the stronger bounds

$$
\sup _{|x|<R}|u(t, x)| \leq K|t|^{b}
$$

for some constants $K, b>0$, whenever $t \in S$. As a consequence, the structure of all the solutions $u(t, x)$ of $(7)$ subjected to (8) (and ressembling the one given by $(5))$ can be completely described by a former result by R. Gérard and H. Tahara stated in [7], Chap. 8.

In [17], the authors study families of formal power series $f(x)$ with real coefficients in double scales of power and logarithmic functions with the shape

$$
f(x)=\lambda x^{\alpha}+\sum_{\beta \in S, \beta>\alpha} \sum_{k \in \mathbb{Z}} a_{\beta, k} x^{\beta}\left(\frac{1}{\log (x)}\right)^{k}
$$

where $\lambda, \alpha>0$ are real numbers and $S$ is contained in a finitely generated additive semi-group in $(0,+\infty)$. Given such an $f$, normal forms for the conjugacy class $\varphi^{-1} \circ f \circ \varphi$ are completely classified in that paper and a so-called embedding theorem is reached. These sets of formal expansions extend the classical formal Dulac series

$$
D(x)=c_{0} x^{\lambda_{0}}+\sum_{i \geq 1} x^{\lambda_{i}} P_{i}(\log (x))
$$

for $c_{0}>0$, increasing sequences $\left\{\lambda_{i}\right\}_{i \geq 0}$ of positive real numbers and polynomials $P_{i}$ with real coefficients, which appear to represent asymptotic expansions at the origin of Poincaré maps $P$ stemming from analytic planar vector fields.

\section{Layout of the main initial value problem and tied up auxiliary problems}

\subsection{Laplace transforms of order $k$ and Fourier inverse maps}

In this short subsection, we include a prefatory material about Laplace transforms and Fourier inverse maps that will be used in the upcoming sections. 
Let $k \geq 1$ be an integer. We recall the definition of the Laplace transform of order $k$ as introduced in [9].

Definition 1 We set $S_{d, \delta}=\left\{\tau \in \mathbb{C}^{*}:|d-\arg (\tau)|<\delta\right\}$ as some unbounded sector with bisecting direction $d \in \mathbb{R}$ and aperture $2 \delta>0$ and $D_{\rho}$ as a disc centered at 0 with radius $\rho>0$. Consider a holomorphic function $w: S_{d, \delta} \cup D_{\rho} \rightarrow \mathbb{C}$ that vanishes at 0 and withstands the bounds : there exist $C>0$ and $K>0$ such that

$$
|w(\tau)| \leq C|\tau| \exp \left(K|\tau|^{k}\right)
$$

for all $\tau \in S_{d, \delta}$. We define the Laplace transform of $w$ of order $k$ in the direction $d$ as the integral transform

$$
\mathcal{L}_{k}^{d}(w)(T)=k \int_{L_{\gamma}} w(u) \exp \left(-\left(\frac{u}{T}\right)^{k}\right) \frac{d u}{u}
$$

along a half-line $L_{\gamma}=[0,+\infty) e^{\sqrt{-1} \gamma} \subset S_{d, \delta} \cup\{0\}$, where $\gamma$ depends on $T$ and is chosen in such a way that $\cos (k(\gamma-\arg (T))) \geq \delta_{1}$, for some fixed real number $\delta_{1}>0$. The function $\mathcal{L}_{k}^{d}(w)(T)$ is well defined, holomorphic and bounded on any sector

$$
S_{d, \theta, R^{1 / k}}=\left\{T \in \mathbb{C}^{*}:|T|<R^{1 / k} \quad, \quad|d-\arg (T)|<\theta / 2\right\},
$$

where $0<\theta<\frac{\pi}{k}+2 \delta$ and $0<R<\delta_{1} / K$.

We remind some useful property : if $w(\tau)$ represents an entire function w.r.t $\tau \in \mathbb{C}$ with the bounds (9), its Laplace transform $\mathcal{L}_{k}^{d}(w)(T)$ does not depend on the direction $d$ in $\mathbb{R}$ and represents a bounded holomorphic function on $D_{R^{1 / k}}$ whose Taylor expansion is represented by the convergent series $X(T)=\sum_{n \geq 1} w_{n} \Gamma\left(\frac{n}{k}\right) T^{n}$ on $D_{R^{1 / k}}$, where $\Gamma(x)$ stands for the Gamma function.

We remind the reader the definition of some family of Banach spaces used for the first time by the author in [14] and introduced in [5].

Definition 2 Let $\beta, \mu \in \mathbb{R}$. We set $E_{(\beta, \mu)}$ as the vector space of continuous functions $h: \mathbb{R} \rightarrow \mathbb{C}$ such that

$$
\|h(m)\|_{(\beta, \mu)}=\sup _{m \in \mathbb{R}}(1+|m|)^{\mu} \exp (\beta|m|)|h(m)|
$$

is finite. The space $E_{(\beta, \mu)}$ endowed with the norm $\|\cdot\|_{(\beta, \mu)}$ becomes a Banach space.

Finally, we restate the definition of the inverse Fourier transform acting on the latter Banach spaces and some of its close at hand formulas relative to derivation and convolution product as expounded in [9].

Definition 3 Let $f \in E_{(\beta, \mu)}$ with $\beta>0, \mu>1$. The inverse Fourier transform of $f$ is given by

$$
\mathcal{F}^{-1}(f)(x)=\frac{1}{(2 \pi)^{1 / 2}} \int_{-\infty}^{+\infty} f(m) \exp (i x m) d m
$$

for all $x \in \mathbb{R}$. The function $\mathcal{F}^{-1}(f)$ extends to an analytic bounded function on the strips

$$
H_{\beta^{\prime}}=\left\{z \in \mathbb{C} /|\operatorname{Im}(z)|<\beta^{\prime}\right\} .
$$

for all given $0<\beta^{\prime}<\beta$.

a) Define the function $m \mapsto \phi(m)=\operatorname{imf}(m)$ which belongs to the space $E_{(\beta, \mu-1)}$. Then, the next identity

$$
\partial_{z} \mathcal{F}^{-1}(f)(z)=\mathcal{F}^{-1}(\phi)(z)
$$


occurs.

b) Take $g \in E_{(\beta, \mu)}$ and set

$$
\psi(m)=\frac{1}{(2 \pi)^{1 / 2}} \int_{-\infty}^{+\infty} f\left(m-m_{1}\right) g\left(m_{1}\right) d m_{1}
$$

as the convolution product of $f$ and $g$. Then, $\psi$ belongs to $E_{(\beta, \mu)}$ and moreover,

$$
\mathcal{F}^{-1}(f)(z) \mathcal{F}^{-1}(g)(z)=\mathcal{F}^{-1}(\psi)(z)
$$

for all $z \in H_{\beta}$.

\subsection{The main problem outlined}

In this subsection, we disclose the principal linear initial value problem under study in this paper. It is stated as follows,

$$
\begin{aligned}
Q\left(\partial_{z}\right) u(t, z, \epsilon)=(\epsilon t)^{d_{D}}\left(t \partial_{t}\right)^{\delta_{D}} R_{D}\left(\partial_{z}\right) & u(t, z, \epsilon) \\
& +\sum_{l=1}^{D-1} \epsilon^{\Delta_{l}} t^{d_{l}}\left(t \partial_{t}\right)^{\delta_{l}} a_{l}(z, \epsilon) R_{l}\left(\partial_{z}\right) u(t, z, \epsilon)+f(t, z, \epsilon)
\end{aligned}
$$

where $D \geq 2$ is some integer, for vanishing initial data $u(0, z, \epsilon) \equiv 0$.

The constants $d_{D}, \delta_{D}, \Delta_{l}, d_{l}$ and $\delta_{l}$ for $1 \leq l \leq D-1$ are positive integers that are submitted to the next list of technical constraints:

1. There exists an integer $k_{1} \geq 1$ such that

$$
d_{D}=\delta_{D} k_{1}, \quad d_{l}>\delta_{l} k_{1}
$$

for all $1 \leq l \leq D-1$.

2. The next three inequalities

$$
\Delta_{l}>0, \quad \Delta_{l}>\delta_{l} k_{1}, \quad k_{1} \delta_{D}-1 \geq k_{1} \delta_{l}
$$

hold for all $1 \leq l \leq D-1$.

The maps $Q(X), R_{D}(X)$ and $R_{l}(X)$ are polynomials with complex coefficients that are subjected to the next two restrictions:

3. The next bounds

$$
\operatorname{deg}(Q) \geq \operatorname{deg}\left(R_{D}\right) \geq \operatorname{deg}\left(R_{l}\right)
$$

hold, for all $1 \leq l \leq D-1$, where $\operatorname{deg}(P)$ denotes the degree of a polynomial $P(X)$.

4. One can select an unbounded sectorial annulus

$$
S_{Q, R_{D}}=\left\{z \in \mathbb{C}^{*} / r_{Q, R_{D}}<|z|,\left|\arg (z)-d_{Q, R_{D}}\right| \leq \eta_{Q, R_{D}}\right\}
$$

with bisecting direction $d_{Q, R_{D}} \in \mathbb{R}$, aperture $\eta_{Q, R_{D}}>0$ and inner radius $r_{Q, R_{D}}>0$ (prescribed later on in the work), for which the next inclusion

$$
\left\{\frac{Q(i m)}{R_{D}(i m)} / m \in \mathbb{R}\right\} \subset S_{Q, R_{D}}
$$

holds true.

The coefficients $a_{l}(z, \epsilon), 1 \leq l \leq D-1$, are built up in the following way. For $1 \leq l \leq D-1$, let $m \mapsto A_{l}(m, \epsilon)$ be maps 
- that belong to the Banach space $E_{(\beta, \mu)}$, for some given real numbers $\beta>0$ and $\mu>1$ that fulfills the restriction

$$
\mu>\operatorname{deg}\left(R_{l}\right)+1
$$

for all $1 \leq l \leq D-1$.

- that depend analytically on $\epsilon$ on a disc $D_{\epsilon_{0}}$ centered at 0 in $\mathbb{C}$ with radius $\epsilon_{0}>0$ and for which a constant $\mathbf{A}_{l, \epsilon_{0}}>0$ can be be singled out with

$$
\sup _{\epsilon \in D_{\epsilon_{0}}}\left\|A_{l}(m, \epsilon)\right\|_{(\beta, \mu)} \leq \mathbf{A}_{l, \epsilon_{0}} .
$$

We set

$$
a_{l}(z, \epsilon)=\mathcal{F}^{-1}\left(m \mapsto A_{l}(m, \epsilon)\right)(z)
$$

for all $1 \leq l \leq D-1$. According to Definition 3, the maps $(z, \epsilon) \mapsto a_{l}(z, \epsilon)$ represent bounded holomorphic maps on the product $H_{\beta^{\prime}} \times D_{\epsilon_{0}}$, for any prescribed $0<\beta^{\prime}<\beta$.

The forcing term $f(t, z, \epsilon)$ is constructed in the next manner. Let $J_{1}, J_{2}$ be given finite subsets of the positive integers $\mathbb{N}^{*}$. For $j_{1} \in J_{1}, j_{2} \in J_{2}$, we denote $m \mapsto \mathcal{F}_{j_{1}, j_{2}}(m, \epsilon)$ maps

- that appertain to the Banach space $E_{(\beta, \mu)}$, for $\beta>0, \mu>1$ given above.

- that rely analytically on $\epsilon$ on the disc $D_{\epsilon_{0}}$, with a constant $\mathbf{F}_{j_{1}, j_{2}, \epsilon_{0}}$ such that

$$
\sup _{\epsilon \in D_{\epsilon_{0}}}\left\|\mathcal{F}_{j_{1}, j_{2}}(m, \epsilon)\right\|_{(\beta, \mu)} \leq \mathbf{F}_{j_{1}, j_{2}, \epsilon_{0}} .
$$

We introduce the next polynomial

$$
\mathcal{F}\left(\tau_{1}, \tau_{2}, m, \epsilon\right)=\sum_{j_{1} \in J_{1}, j_{2} \in J_{2}} \mathcal{F}_{j_{1}, j_{2}}(m, \epsilon) \tau_{1}^{j_{1}} \tau_{2}^{j_{2}}
$$

in the variables $\tau_{1}, \tau_{2}$, with coefficients in $E_{(\beta, \mu)}$ that depends analytically in $\epsilon$ on $D_{\epsilon_{0}}$. We consider some given real number $a>0$ and we set

$$
\begin{aligned}
& F_{\pi, a}\left(u_{1}, u_{2}, z, \epsilon\right) \\
& \quad=\frac{k_{1}}{(2 \pi)^{1 / 2}} \int_{L_{d_{1}}} \int_{L_{\pi, a}} \int_{-\infty}^{+\infty} \mathcal{F}\left(\tau_{1}, \tau_{2}, m, \epsilon\right) \exp \left(-\left(\frac{\tau_{1}}{u_{1}}\right)^{k_{1}}-\left(\frac{\tau_{2}}{u_{2}}\right)\right) e^{i z m} \frac{d \tau_{1}}{\tau_{1}} \frac{d \tau_{2}}{\tau_{2}} d m
\end{aligned}
$$

where $L_{d_{1}}=[0, \infty) e^{\sqrt{-1} d_{1}}$ stands for a halfline in direction $d_{1} \in \mathbb{R}$, which depends on $\tau_{1}$ in a way that $\cos \left(k_{1}\left(d_{1}-\arg \left(u_{1}\right)\right)\right)$ remains strictly positive and where $L_{\pi, a}=[0, a] e^{\sqrt{-1} \pi}$ stands for the segment $[-a, 0]$.

Owing to Definition 1, the map $F_{\pi, a}$ can be written in the form of a polynomial in $u_{1}$,

$$
F_{\pi, a}\left(u_{1}, u_{2}, z, \epsilon\right)=\sum_{j_{1} \in J_{1}} F_{\pi, a, j_{1}}\left(u_{2}, z, \epsilon\right) \Gamma\left(\frac{j_{1}}{k_{1}}\right) u_{1}^{j_{1}}
$$

whose coefficients $\left(u_{2}, z, \epsilon\right) \mapsto F_{\pi, a, j_{1}}\left(u_{2}, z, \epsilon\right)$ are holomorphic on $\mathbb{C}^{*} \times H_{\beta} \times D_{\epsilon_{0}}$. Notice that the expression $F_{\pi, a}$ does not depend on the choice of the direction $d_{1}$. An explicit expression of the maps $F_{\pi, a, j_{1}}$ will be disclosed later on in the work (see Section 5, Proposition 10, Lemma 6). 
Due to Definition 1, we observe that when the radius $a>0$ tends to infinity, each partial map $u_{2} \mapsto F_{\pi, a, j_{1}}\left(u_{2}, z, \epsilon\right)$ becomes close to the polynomial in $u_{2}$,

$$
\sum_{j_{2} \in J_{2}} \mathcal{F}^{-1}\left(m \mapsto \mathcal{F}_{j_{1}, j_{2}}(m, \epsilon)\right)(z) \Gamma\left(j_{2}\right) u_{2}^{j_{2}}
$$

with bounded holomorphic coefficients on $H_{\beta^{\prime}} \times D_{\epsilon_{0}}$, for any $0 \leq \beta^{\prime}<\beta$, provided that the variable $u_{2}$ satisfies

$$
\arg \left(u_{2}\right) \in\left(-\pi,-\frac{\pi}{2}-\delta\right) \cup\left(\frac{\pi}{2}+\delta, \pi\right] \bmod (2 \pi)
$$

for some small $\delta>0$. The forcing term $f(t, z, \epsilon)$ is defined by the logarithmic type function

$$
f(t, z, \epsilon)=F_{\pi, a}\left(\epsilon t, \frac{1}{\log (\epsilon t)}, z, \epsilon\right) .
$$

Here $\log (z)$ stands for the principal value of the logarithm, namely $\log (z)=\ln |z|+\sqrt{-1} \arg (z)$ with $\arg (z) \in(-\pi, \pi)$. By construction, we notice that

$$
\arg \left(\frac{1}{\log (z)}\right)=-\arg (\log (z)) \in\left(-\pi,-\frac{\pi}{2}-\delta\right) \cup\left(\frac{\pi}{2}+\delta, \pi\right] \bmod (2 \pi)
$$

provided that $|z|$ is small enough with $z \notin(-\infty, 0]$, for any given small $\delta>0$.

As a result, from the expansion (21), one checks that $f(t, z, \epsilon)$ represents a holomorphic function in $z \in H_{\beta^{\prime}}$ and $t, \epsilon$ for $t \in D$ (some small disc centered at 0 ) and $\epsilon \in D_{\epsilon_{0}} \backslash\{0\}$, provided that $\epsilon t \notin(-\infty, 0]$ and as long as $\epsilon_{0}>0$ is taken small enough. Furthermore, from the discussion above, when the radius $a>0$ is taken large enough, the forcing term $f(t, z, \epsilon)$ becomes proximate to the explicit logarithmic type function in $t, \epsilon$

$$
f_{\infty}(t, z, \epsilon)=\sum_{j_{1} \in J_{1}, j_{2} \in J_{2}} \mathcal{F}^{-1}\left(m \mapsto \mathcal{F}_{j_{1}, j_{2}}(m, \epsilon)\right)(z) \Gamma\left(\frac{j_{1}}{k_{1}}\right) \Gamma\left(j_{2}\right)(\epsilon t)^{j_{1}}\left(\frac{1}{\log (\epsilon t)}\right)^{j_{2}} .
$$

Notice that each single piece $\psi_{j_{1}, j_{2}}(t, \epsilon)=(\epsilon t)^{j_{1}} /(\log (\epsilon t))^{j_{2}}$, for $j_{1} \in J_{1}, j_{2} \in J_{2}$ solves an explicit nonlinear ordinary differential equation which is both singularly perturbed in the parameter $\epsilon$ and possesses a differential operator of first order with irregular type, namely

$$
\epsilon^{j_{1} / j_{2}} t^{1+\frac{j_{1}}{j_{2}}} \partial_{t} \psi_{j_{1}, j_{2}}(t, \epsilon)=j_{1} \epsilon^{j_{1} / j_{2}} t^{j_{1} / j_{2}} \psi_{j_{1}, j_{2}}(t, \epsilon)-j_{2}\left(\psi_{j_{1}, j_{2}}(t, \epsilon)\right)^{1+\frac{1}{j_{2}}}
$$

for all $t, \epsilon \in \mathbb{C}$ such that $\epsilon t \notin(-\infty, 0]$. The reason for which we need to restrict ourselves to a truncated Laplace transform in the variable $u_{2}$ for the expression (20) instead of a complete Laplace integral $(a=+\infty)$ will be expounded later on in the work.

\subsection{A family of related initial value problems}

In this subsection, we reduce the study of the main problem (11) to the analysis of a set of auxiliary problems which involves four independent complex variables.

We plan to seek for solutions $u(t, z, \epsilon)$ to the equation (11) with vanishing initial data at $t=0$ of the form

$$
u(t, z, \epsilon)=U_{\pi}\left(\epsilon t, \frac{1}{\log (\epsilon t)}, z, \epsilon\right)
$$

for some expression $U_{\pi}\left(u_{1}, u_{2}, z, \epsilon\right)$ in the four independent variables $u_{1}, u_{2}, z$ and $\epsilon$. 
Following the usual chain rule (applied at a formal level at this stage of the work), the next computation holds

$$
\begin{aligned}
t \partial_{t} u(t, z, \epsilon)=t\left(\partial_{t}(\epsilon t)\right)\left(\partial_{u_{1}} U_{\pi}\right)\left(\epsilon t, \frac{1}{\log (\epsilon t)}, z, \epsilon\right)+ & t \partial_{t}\left(\frac{1}{\log (\epsilon t)}\right)\left(\partial_{u_{2}} U_{\pi}\right)\left(\epsilon t, \frac{1}{\log (\epsilon t)}, z, \epsilon\right) \\
& =\left(\left(u_{1} \partial_{u_{1}}-u_{2}^{2} \partial_{u_{2}}\right) U_{\pi}\right)\left(\epsilon t, \frac{1}{\log (\epsilon t)}, z, \epsilon\right)
\end{aligned}
$$

As a result, the expression $u(t, z, \epsilon)$ (formally) solves the equation (11) with vanishing data at $t=0$ if the expression $U_{\pi}\left(u_{1}, u_{2}, z, \epsilon\right)$ solves the next equation

$$
\begin{aligned}
Q\left(\partial_{z}\right) U_{\pi} & \left(u_{1}, u_{2}, z, \epsilon\right)=u_{1}^{d_{D}}\left(u_{1} \partial_{u_{1}}-u_{2}^{2} \partial_{u_{2}}\right)^{\delta_{D}} R_{D}\left(\partial_{z}\right) U_{\pi}\left(u_{1}, u_{2}, z, \epsilon\right) \\
& +\sum_{l=1}^{D-1} \epsilon^{\Delta_{l}-d_{l}} u_{1}^{d_{l}}\left(u_{1} \partial_{u_{1}}-u_{2}^{2} \partial_{u_{2}}\right)^{\delta_{l}} a_{l}(z, \epsilon) R_{l}\left(\partial_{z}\right) U_{\pi}\left(u_{1}, u_{2}, z, \epsilon\right)+F_{\pi, a}\left(u_{1}, u_{2}, z, \epsilon\right)
\end{aligned}
$$

for given vanishing initial data $U_{\pi}(0,0, z, \epsilon) \equiv 0$. Here the symbol $\left(u_{1} \partial_{u_{1}}-u_{2}^{2} \partial_{u_{2}}\right)^{h}$ stands for the $h$-iterate of the differential operator $u_{1} \partial_{u_{1}}-u_{2}^{2} \partial_{u_{2}}$ for any given integer $h \geq 1$.

In order to be able to build genuine solutions to (11) and furthermore to study their asymptotic properties as $\epsilon$ tends to 0 , we need to examine a more general family of related problems stated as follows.

For any given direction $d_{2} \in \mathbb{R}$, we define

$$
\begin{aligned}
& F_{d_{2}, a}\left(u_{1}, u_{2}, z, \epsilon\right) \\
& \quad=\frac{k_{1}}{(2 \pi)^{1 / 2}} \int_{L_{d_{1}}} \int_{L_{d_{2}, a}} \int_{-\infty}^{+\infty} \mathcal{F}\left(\tau_{1}, \tau_{2}, m, \epsilon\right) \exp \left(-\left(\frac{\tau_{1}}{u_{1}}\right)^{k_{1}}-\left(\frac{\tau_{2}}{u_{2}}\right)\right) e^{i z m} \frac{d \tau_{1}}{\tau_{1}} \frac{d \tau_{2}}{\tau_{2}} d m
\end{aligned}
$$

where the direction $d_{1} \in \mathbb{R}$ depends on $\tau_{1}$ in a way that $\cos \left(k_{1}\left(d_{1}-\arg \left(u_{1}\right)\right)\right)$ remains strictly positive and where $L_{d_{2}, a}=[0, a] e^{\sqrt{-1} d_{2}}$ stands for a segment of length $a$ in direction $d_{2}$. Owing to Definition 1, we notice that the expression $F_{d_{2}, a}$ does not rely on the direction $d_{1}$.

We consider the next equation

$$
\begin{aligned}
& Q\left(\partial_{z}\right) U_{d_{2}}\left(u_{1}, u_{2}, z, \epsilon\right)=u_{1}^{d_{D}}\left(u_{1} \partial_{u_{1}}-u_{2}^{2} \partial_{u_{2}}\right)^{\delta_{D}} R_{D}\left(\partial_{z}\right) U_{d_{2}}\left(u_{1}, u_{2}, z, \epsilon\right) \\
& \quad+\sum_{l=1}^{D-1} \epsilon^{\Delta_{l}-d_{l}} u_{1}^{d_{l}}\left(u_{1} \partial_{u_{1}}-u_{2}^{2} \partial_{u_{2}}\right)^{\delta_{l}} a_{l}(z, \epsilon) R_{l}\left(\partial_{z}\right) U_{d_{2}}\left(u_{1}, u_{2}, z, \epsilon\right)+F_{d_{2}, a}\left(u_{1}, u_{2}, z, \epsilon\right)
\end{aligned}
$$

with prescribed vanishing initial data $U_{d_{2}}(0,0, z, \epsilon) \equiv 0$.

\section{Construction of analytic solutions to the set of related initial value problems}

In this section, we plan to construct a family of analytic solutions $U_{d_{1}, d_{2}}\left(u_{1}, u_{2}, z, \epsilon\right)$ to the auxiliary problem (30) obtained for well chosen directions $d_{1} \in \mathbb{R}$, for any given direction $d_{2} \in \mathbb{R}$. 


\subsection{The shape of the analytic solutions and associated convolution equation}

For any given direction $d_{2} \in \mathbb{R}$, we search for a family of solutions to (30) in the form of a double Laplace transform and inverse Fourier integral

$$
\begin{aligned}
& U_{d_{1}, d_{2}}\left(u_{1}, u_{2}, z, \epsilon\right) \\
& =\frac{k_{1}}{(2 \pi)^{1 / 2}} \int_{L_{d_{1}}} \int_{L_{d_{2}, a}} \int_{-\infty}^{+\infty} \omega_{d_{1}}\left(\tau_{1}, \tau_{2}, m, \epsilon\right) \exp \left(-\left(\frac{\tau_{1}}{u_{1}}\right)^{k_{1}}-\left(\frac{\tau_{2}}{u_{2}}\right)\right) e^{i z m} \frac{d \tau_{1}}{\tau_{1}} \frac{d \tau_{2}}{\tau_{2}} d m .
\end{aligned}
$$

Here, we assume that the so-called Borel-Fourier map $\omega_{d_{1}}$ belongs to a Banach space of functions denoted $F_{\left(\nu, \beta, \mu, k_{1}, \rho, a, \epsilon\right)}^{d_{1}}$ described in the next definition.

Definition 4 Let $\epsilon_{0}, \beta, \mu, k_{1}, a$ be the constants prescribed in Subsection 2.2. Let $\epsilon \in D_{\epsilon_{0}} \backslash\{0\}$ and $\nu, \rho>0$ be given real numbers. We set as $S_{d_{1}}$ an unbounded sector centered at 0 with bisecting direction $d_{1} \in \mathbb{R}$. We denote $F_{\left(\nu, \beta, \mu, k_{1}, \rho, a, \epsilon\right)}^{d_{1}}$ the vector space of all continuous maps $\left(\tau_{1}, \tau_{2}, m\right) \mapsto h\left(\tau_{1}, \tau_{2}, m\right)$ on $\left(S_{d_{1}} \cup D_{\rho}\right) \times D_{a} \times \mathbb{R}$, holomorphic w.r.t $\tau_{1}, \tau_{2}$ on $\left(S_{d_{1}} \cup D_{\rho}\right) \times D_{a}$, such that the norm

$$
\begin{aligned}
& \left\|h\left(\tau_{1}, \tau_{2}, m\right)\right\|_{\left(\nu, \beta, \mu, k_{1}, \rho, a, \epsilon\right)} \\
& \quad=\sup _{\substack{\tau_{1} \in S_{d_{1} \cup D_{\rho}, \tau_{2} \in D_{a}} \\
m \in \mathbb{R}}}(1+|m|)^{\mu} \exp (\beta|m|)\left|\frac{\epsilon}{\tau_{1}}\right| \exp \left(-\left.\nu\left|\frac{\tau_{1}}{\epsilon}\right|\right|^{k_{1}}\right) \frac{1}{\left|\tau_{2}\right|}\left|h\left(\tau_{1}, \tau_{2}, m\right)\right|
\end{aligned}
$$

is finite. The vector space $F_{\left(\nu, \beta, \mu, k_{1}, \rho, a, \epsilon\right)}^{d_{1}}$ equipped with the norm $\|\cdot\|_{\left(\nu, \beta, \mu, k_{1}, \rho, a, \epsilon\right)}$ becomes a Banach space.

Remark. Similar Banach spaces that involve functions with two complex and one real variables have been recently introduced in the works [11], [12] by A. Lastra and the author.

Our main task within this subsection is to derive some convolution equation fulfilled by the Borel-Fourier map $\omega_{d_{1}}$.

We recall some features of the Laplace transform under the action of multiplication by a monomial and differential operators already stated in our foregoing work [9]. A detailed proof of the formulas stated in the forthcoming lemma can be found in the work [10], Lemma 2.

Lemma 1 Let us assume that the map $\omega_{d_{1}}$ appertains to the space $F_{\left(\nu, \beta, \mu, k_{1}, \rho, a, \epsilon\right)}^{d_{1}}$. Then, the next identities hold.

1. The action of the differential operator $u_{1}^{k_{1}+1} \partial_{u_{1}}$ on the integral representation (31) writes

$$
\begin{aligned}
& u_{1}^{k_{1}+1} \partial_{u_{1}} U_{d_{1}, d_{2}}\left(u_{1}, u_{2}, z, \epsilon\right) \\
= & \frac{k_{1}}{(2 \pi)^{1 / 2}} \int_{L_{d_{1}}} \int_{L_{d_{2}, a}} \int_{-\infty}^{+\infty}\left\{k_{1} \tau_{1}^{k_{1}} \omega_{d_{1}}\left(\tau_{1}, \tau_{2}, m, \epsilon\right)\right\} \exp \left(-\left(\frac{\tau_{1}}{u_{1}}\right)^{k_{1}}-\left(\frac{\tau_{2}}{u_{2}}\right)\right) e^{i z m} \frac{d \tau_{1}}{\tau_{1}} \frac{d \tau_{2}}{\tau_{2}} d m .
\end{aligned}
$$

2. Let $m^{\prime} \geq 1$ be an integer. The multiplication by $u_{1}^{m^{\prime}}$ acting on (31) is expressed through

$$
\begin{aligned}
& u_{1}^{m^{\prime}} U_{d_{1}, d_{2}}\left(u_{1}, u_{2}, z, \epsilon\right) \\
& =\frac{k_{1}}{(2 \pi)^{1 / 2}} \int_{L_{d_{1}}} \int_{L_{d_{2}, a}} \int_{-\infty}^{+\infty}\left\{\frac{\tau_{1}^{k_{1}}}{\Gamma\left(\frac{m^{\prime}}{k_{1}}\right)} \int_{0}^{\tau_{1}^{k_{1}}}\left(\tau_{1}^{k_{1}}-s_{1}\right)^{\frac{m^{\prime}}{k_{1}}-1} \omega_{d_{1}}\left(s_{1}^{1 / k_{1}}, \tau_{2}, m, \epsilon\right) \frac{d s_{1}}{s_{1}}\right\} \\
& \times \exp \left(-\left(\frac{\tau_{1}}{u_{1}}\right)^{k_{1}}-\left(\frac{\tau_{2}}{u_{2}}\right)\right) e^{i z m} \frac{d \tau_{1}}{\tau_{1}} \frac{d \tau_{2}}{\tau_{2}} d m .
\end{aligned}
$$


3. The differentiel operator $u_{2} \partial_{u_{2}}$ applies on (31) by means of

$$
\begin{aligned}
& u_{2}^{2} \partial_{u_{2}} U_{d_{1}, d_{2}}\left(u_{1}, u_{2}, z, \epsilon\right) \\
& \quad=\frac{k_{1}}{(2 \pi)^{1 / 2}} \int_{L_{d_{1}}} \int_{L_{d_{2}, a}} \int_{-\infty}^{+\infty}\left\{\tau_{2} \omega_{d_{1}}\left(\tau_{1}, \tau_{2}, m, \epsilon\right)\right\} \exp \left(-\left(\frac{\tau_{1}}{u_{1}}\right)^{k_{1}}-\left(\frac{\tau_{2}}{u_{2}}\right)\right) e^{i z m} \frac{d \tau_{1}}{\tau_{1}} \frac{d \tau_{2}}{\tau_{2}} d m
\end{aligned}
$$

In the next steps, we plan to express all the differential operators acting on the variables $u_{1}, u_{2}$ that appear in the related initial value problem (30) by the agency of the basic operator listed in the above lemma.

At first, since the operators $u_{1} \partial_{u_{1}}$ and $u_{2}^{2} \partial_{u_{2}}$ commute to each other, one can rewrite (30) by way of the classical binomial formula as

$$
\begin{aligned}
& Q\left(\partial_{z}\right) U_{d_{2}}\left(u_{1}, u_{2}, z, \epsilon\right)=u_{1}^{d_{D}} \\
& \times \sum_{p_{1}+p_{2}=\delta_{D}} \frac{\delta_{D} !}{p_{1} ! p_{2} !}\left(u_{1} \partial_{u_{1}}\right)^{p_{1}}(-1)^{p_{2}}\left(u_{2}^{2} \partial_{u_{2}}\right)^{p_{2}} R_{D}\left(\partial_{z}\right) U_{d_{2}}\left(u_{1}, u_{2}, z, \epsilon\right) \\
& +\sum_{l=1}^{D-1} \epsilon^{\Delta_{l}-d_{l}} u_{1}^{d_{l}} \times \sum_{p_{1}+p_{2}=\delta_{l}} \frac{\delta_{l} !}{p_{1} ! p_{2} !}\left(u_{1} \partial_{u_{1}}\right)^{p_{1}}(-1)^{p_{2}}\left(u_{2}^{2} \partial_{u_{2}}\right)^{p_{2}} a_{l}(z, \epsilon) R_{l}\left(\partial_{z}\right) U_{d_{2}}\left(u_{1}, u_{2}, z, \epsilon\right) \\
& +F_{d_{2}, a}\left(u_{1}, u_{2}, z, \epsilon\right)
\end{aligned}
$$

for given initial data $U_{d_{2}}(0,0, z, \epsilon) \equiv 0$.

In a second step, we apply a useful lemma already stated in the previous work of A. Lastra and the author, [13] which provide expansions for the iterations of the basic fuchsian operator $u_{1} \partial_{u_{1}}$.

Lemma 2 For all integer $p_{1} \geq 1$, there exist positive integers $a_{q, p_{1}} \geq 1$, for $1 \leq q \leq p_{1}$, such that

$$
\left(u_{1} \partial_{u_{1}}\right)^{p_{1}}=\sum_{q=1}^{p_{1}} a_{q, p_{1}} u_{1}^{q} \partial_{u_{1}}^{q}
$$

with $a_{1, p_{1}}=a_{p_{1}, p_{1}}=1$.

By dint of this lemma, we can recast the last equation (35) in the form

$$
\begin{aligned}
& Q\left(\partial_{z}\right) U_{d_{2}}\left(u_{1}, u_{2}, z, \epsilon\right)=u_{1}^{d_{D}} \times\left[(-1)^{\delta_{D}}\left(u_{2}^{2} \partial_{u_{2}}\right)^{\delta_{D}} R_{D}\left(\partial_{z}\right) U_{d_{2}}\left(u_{1}, u_{2}, z, \epsilon\right)\right. \\
& \left.+\sum_{\substack{p_{1}+p_{2}=\delta_{D} \\
1 \leq p_{1} \leq \delta_{D}}} \frac{\delta_{D} !}{p_{1} ! p_{2} !}\left(\sum_{q=1}^{p_{1}} a_{q, p_{1}} u_{1}^{q} \partial_{u_{1}}^{q}\right)(-1)^{p_{2}}\left(u_{2}^{2} \partial_{u_{2}}\right)^{p_{2}} R_{D}\left(\partial_{z}\right) U_{d_{2}}\left(u_{1}, u_{2}, z, \epsilon\right)\right] \\
& +\sum_{l=1}^{D-1} \epsilon^{\Delta_{l}-d_{l}} u_{1}^{d_{l}} \times\left[(-1)^{\delta_{l}}\left(u_{2}^{2} \partial_{u_{2}}\right)^{\delta_{l}} a_{l}(z, \epsilon) R_{l}\left(\partial_{z}\right) U_{d_{2}}\left(u_{1}, u_{2}, z, \epsilon\right)\right. \\
& \left.+\sum_{\substack{p_{1}+p_{2}=\delta_{l} \\
1 \leq p_{1} \leq \delta_{l}}} \frac{\delta_{l} !}{p_{1} ! p_{2} !}\left(\sum_{q=1}^{p_{1}} a_{q, p_{1}} u_{1}^{q} \partial_{u_{1}}^{q}\right)(-1)^{p_{2}}\left(u_{2}^{2} \partial_{u_{2}}\right)^{p_{2}} a_{l}(z, \epsilon) R_{l}\left(\partial_{z}\right) U_{d_{2}}\left(u_{1}, u_{2}, z, \epsilon\right)\right]+F_{d_{2}, a}\left(u_{1}, u_{2}, z, \epsilon\right)
\end{aligned}
$$

In a last undertaking, we bring into play a helpful formula introduced in the work [22] and which appears in many papers of the author and his colleagues, going back to its earliest occurrence in [9], that is stated as follows. 
Lemma 3 Let $k_{1}, \delta \geq 1$ be integers. One can single out real numbers $A_{\delta, p}$, for $1 \leq p \leq \delta-1$ such that

$$
u_{1}^{\delta\left(k_{1}+1\right)} \partial_{u_{1}}^{\delta}=\left(u_{1}^{k_{1}+1} \partial_{u_{1}}\right)^{\delta}+\sum_{1 \leq p \leq \delta-1} A_{\delta, p} u_{1}^{k_{1}(\delta-p)}\left(u_{1}^{k_{1}+1} \partial_{u_{1}}\right)^{p}
$$

where, by convention, we assume that the sum $\sum_{1 \leq p \leq \delta-1}[\ldots]$ vanishes when $\delta=1$ in (37).

Owing to the conditions (12) imposed on the integers $k_{1}$ and $d_{l}, \delta_{l}$, for $1 \leq l \leq D$, we deduce the next decompositions. First, we rewrite the equality in (12), as

$$
d_{D}+\delta_{D}=\delta_{D}\left(k_{1}+1\right)
$$

from which we deduce the existence of integers $d_{D, q} \geq 1$, for $1 \leq q<\delta_{D}$ such that

$$
d_{D}+q=q\left(k_{1}+1\right)+d_{D, q}
$$

as long as $1 \leq q<\delta_{D}$. Furthermore, the inequality in (12) gives rise to integers $d_{l, q} \geq 1$, for $1 \leq l \leq D-1,1 \leq q \leq \delta_{l}$ for which

$$
d_{l}+q=q\left(k_{1}+1\right)+d_{l, q}
$$

whenever $1 \leq l \leq D-1,1 \leq q \leq \delta_{l}$. These last three formulas (38), (39), (40) together with (37) beget the next final remodeling of (30) defined as

$$
\begin{aligned}
& Q\left(\partial_{z}\right) U_{d_{2}}\left(u_{1}, u_{2}, z, \epsilon\right)=u_{1}^{d_{D}}(-1)^{\delta_{D}}\left(u_{2}^{2} \partial_{u_{2}}\right)^{\delta_{D}} R_{D}\left(\partial_{z}\right) U_{d_{2}}\left(u_{1}, u_{2}, z, \epsilon\right) \\
& +\sum_{\substack{p_{1}+p_{2}=\delta_{D} \\
1 \leq p_{1}<\delta_{D}}} \frac{\delta_{D} !}{p_{1} ! p_{2} !}\left(\sum_{q=1}^{p_{1}} a_{q, p_{1}} u_{1}^{d_{D, q}}\left[\left(u_{1}^{k_{1}+1} \partial_{u_{1}}\right)^{q}+\sum_{1 \leq p \leq q-1} A_{q, p} u_{1}^{k_{1}(q-p)}\left(u_{1}^{k_{1}+1} \partial_{u_{1}}\right)^{p}\right]\right) \\
& \times(-1)^{p_{2}}\left(u_{2}^{2} \partial_{u_{2}}\right)^{p_{2}} R_{D}\left(\partial_{z}\right) U_{d_{2}}\left(u_{1}, u_{2}, z, \epsilon\right)+\sum_{q=1}^{\delta_{D}-1} a_{q, \delta_{D}} u_{1}^{d_{D, q}}\left[\left(u_{1}^{k_{1}+1} \partial_{u_{1}}\right)^{q}\right. \\
& \left.+\sum_{1 \leq p \leq q-1} A_{q, p} u_{1}^{k_{1}(q-p)}\left(u_{1}^{k_{1}+1} \partial_{u_{1}}\right)^{p}\right] R_{D}\left(\partial_{z}\right) U_{d_{2}}\left(u_{1}, u_{2}, z, \epsilon\right)+ \\
& {\left[\left(u_{1}^{k_{1}+1} \partial_{u_{1}}\right)^{\delta_{D}}+\sum_{1 \leq p \leq \delta_{D}-1} A_{\delta_{D}, p} u_{1}^{k_{1}\left(\delta_{D}-p\right)}\left(u_{1}^{k_{1}+1} \partial_{u_{1}}\right)^{p}\right] R_{D}\left(\partial_{z}\right) U_{d_{2}}\left(u_{1}, u_{2}, z, \epsilon\right)} \\
& +\sum_{l=1}^{D-1} \epsilon^{\Delta_{l}-d_{l}}\left[u_{1}^{d_{l}}(-1)^{\delta_{l}}\left(u_{2}^{2} \partial_{u_{2}}\right)^{\delta_{l}} a_{l}(z, \epsilon) R_{l}\left(\partial_{z}\right) U_{d_{2}}\left(u_{1}, u_{2}, z, \epsilon\right)\right. \\
& +\sum_{\substack{p_{1}+p_{2}=\delta_{l} \\
1 \leq p_{1} \leq \delta_{l}}} \frac{\delta_{l} !}{p_{1} ! p_{2} !}\left(\sum_{q=1}^{p_{1}} a_{q, p_{1}} u_{1}^{d_{l, q}}\left[\left(u_{1}^{k_{1}+1} \partial_{u_{1}}\right)^{q}+\sum_{1 \leq p \leq q-1} A_{q, p} u_{1}^{k_{1}(q-p)}\left(u_{1}^{k_{1}+1} \partial_{u_{1}}\right)^{p}\right]\right. \\
& \left.\left.\times(-1)^{p_{2}}\left(u_{2}^{2} \partial_{u_{2}}\right)^{p_{2}} a_{l}(z, \epsilon) R_{l}\left(\partial_{z}\right) U_{d_{2}}\left(u_{1}, u_{2}, z, \epsilon\right)\right)\right]+F_{d_{2}, a}\left(u_{1}, u_{2}, z, \epsilon\right)
\end{aligned}
$$

for prescribed vanishing data $U_{d_{2}}(0,0, z, \epsilon) \equiv 0$.

On the basis of the identities displayed in Definition 3 and Lemma 1, this last way (41) of rephrasing (30) allows us to reach the following statement. 
The map $U_{d_{1}, d_{2}}\left(u_{1}, u_{2}, z, \epsilon\right)$ given by (31) solves the equation (41) under the additional vanishing condition $U_{d_{1}, d_{2}}(0,0, z, \epsilon) \equiv 0$ if the Borel map $\omega_{d_{1}}\left(\tau_{1}, \tau_{2}, m, \epsilon\right)$ fulfills the next convolution equation

$$
Q(i m) \omega_{d_{1}}\left(\tau_{1}, \tau_{2}, m, \epsilon\right)
$$

$$
\begin{gathered}
=\frac{\tau_{1}^{k_{1}}}{\Gamma\left(\frac{d_{D}}{k_{1}}\right)} \int_{0}^{\tau_{1}^{k_{1}}}\left(\tau_{1}^{k_{1}}-s_{1}\right)^{\frac{d_{D}}{k_{1}}-1} \tau_{2}^{\delta_{D}}(-1)^{\delta_{D}} R_{D}(i m) \omega_{d_{1}}\left(s_{1}^{1 / k_{1}}, \tau_{2}, m, \epsilon\right) \frac{d s_{1}}{s_{1}} \\
+\sum_{\substack{p_{1}+p_{2}=\delta_{D} \\
1 \leq p_{1}<\delta_{D}}} \frac{\delta_{D} !}{p_{1} ! p_{2} !}\left(\sum _ { q = 1 } ^ { p _ { 1 } } a _ { q , p _ { 1 } } \left[\frac{\tau_{1}^{k_{1}}}{\Gamma\left(\frac{d_{D, q}}{k_{1}}\right)} \int_{0}^{\tau_{1}^{k_{1}}}\left(\tau_{1}^{k_{1}}-s_{1}\right)^{\frac{d_{D, q}}{k_{1}}-1}\left(k_{1}\left(s_{1}^{1 / k_{1}}\right)^{k_{1}}\right)^{q} \omega_{d_{1}}\left(s_{1}^{1 / k_{1}}, \tau_{2}, m, \epsilon\right) \frac{d s_{1}}{s_{1}}\right.\right.
\end{gathered}
$$$$
\left.\left.+\sum_{1 \leq p \leq q-1} A_{q, p} \frac{\tau_{1}^{k_{1}}}{\Gamma\left(\frac{d_{D, q}+k_{1}(q-p)}{k_{1}}\right)} \int_{0}^{\tau_{1}^{k_{1}}}\left(\tau_{1}^{k_{1}}-s_{1}\right)^{\frac{d_{D, q}+k_{1}(q-p)}{k_{1}}-1}\left(k_{1}\left(s_{1}^{1 / k_{1}}\right)^{k_{1}}\right)^{p} \omega_{d_{1}}\left(s_{1}^{1 / k_{1}}, \tau_{2}, m, \epsilon\right) \frac{d s_{1}}{s_{1}}\right]\right)
$$$$
\times(-1)^{p_{2}} \tau_{2}^{p_{2}} R_{D}(i m)+\sum_{q=1}^{\delta_{D}-1} a_{q, \delta_{D}}\left[\frac{\tau_{1}^{k_{1}}}{\Gamma\left(\frac{d_{D, q}}{k_{1}}\right)} \int_{0}^{\tau_{1}^{k_{1}}}\left(\tau_{1}^{k_{1}}-s_{1}\right)^{\frac{d_{D, q}}{k_{1}}-1}\left(k_{1}\left(s_{1}^{1 / k_{1}}\right)^{k_{1}}\right)^{q} \omega_{d_{1}}\left(s_{1}^{1 / k_{1}}, \tau_{2}, m, \epsilon\right) \frac{d s_{1}}{s_{1}}\right.
$$$$
\left.+\sum_{1 \leq p \leq q-1} A_{q, p} \frac{\tau_{1}^{k_{1}}}{\Gamma\left(\frac{d_{D, q}+k_{1}(q-p)}{k_{1}}\right)} \int_{0}^{\tau_{1}^{k_{1}}}\left(\tau_{1}^{k_{1}}-s_{1}\right)^{\frac{d_{D, q}+k_{1}(q-p)}{k_{1}}-1}\left(k_{1}\left(s_{1}^{1 / k_{1}}\right)^{k_{1}}\right)^{p} \omega_{d_{1}}\left(s_{1}^{1 / k_{1}}, \tau_{2}, m, \epsilon\right) \frac{d s_{1}}{s_{1}}\right]
$$$$
\times R_{D}(i m)+\left(k_{1} \tau_{1}^{k_{1}}\right)^{\delta_{D}} R_{D}(i m) \omega_{d_{1}}\left(\tau_{1}, \tau_{2}, m, \epsilon\right)
$$

$+\sum_{1 \leq p \leq \delta_{D}-1} A_{\delta_{D}, p} \frac{\tau_{1}^{k_{1}}}{\Gamma\left(\frac{k_{1}\left(\delta_{D}-p\right)}{k_{1}}\right)} \int_{0}^{\tau_{1}^{k_{1}}}\left(\tau_{1}^{k_{1}}-s_{1}\right)^{\frac{k_{1}\left(\delta_{D}-p\right)}{k_{1}}-1}\left(k_{1}\left(s_{1}^{1 / k_{1}}\right)^{k_{1}}\right)^{p} \omega_{d_{1}}\left(s_{1}^{1 / k_{1}}, \tau_{2}, m, \epsilon\right) \frac{d s_{1}}{s_{1}} R_{D}(i m)$

$$
+\sum_{l=1}^{D-1} \epsilon^{\Delta_{l}-d_{l}}\left[\frac{\tau_{1}^{k_{1}}}{\Gamma\left(\frac{d_{l}}{k_{1}}\right)} \int_{0}^{\tau_{1}^{k_{1}}}\left(\tau_{1}^{k_{1}}-s_{1}\right)^{\frac{d_{l}}{k_{1}}-1} \tau_{2}^{\delta_{l}}(-1)^{\delta_{l}}\right.
$$

$$
\times\left(\frac{1}{(2 \pi)^{1 / 2}} \int_{-\infty}^{+\infty} A_{l}\left(m-m_{1}, \epsilon\right) R_{l}\left(i m_{1}\right) \omega_{d_{1}}\left(s_{1}^{1 / k_{1}}, \tau_{2}, m_{1}, \epsilon\right) d m_{1}\right) \frac{d s_{1}}{s_{1}}+\sum_{\substack{p_{1}+p_{2}=\delta_{l} \\ 1 \leq p_{1} \leq \delta_{l}}} \frac{\delta_{l} !}{p_{1} ! p_{2} !}\left(\sum_{q=1}^{p_{1}} a_{q, p_{1}}\right.
$$

$$
\times\left[\frac{\tau_{1}^{k_{1}}}{\Gamma\left(\frac{d_{l, q}}{k_{1}}\right)} \int_{0}^{\tau_{1}^{k_{1}}}\left(\tau_{1}^{k_{1}}-s_{1}\right)^{\frac{d_{l, q}}{k_{1}}-1}(-1)^{p_{2}} \tau_{2}^{p_{2}}\left(k_{1}\left(s_{1}^{1 / k_{1}}\right)^{k_{1}}\right)^{q}\right.
$$

$$
\begin{gathered}
\times\left(\frac{1}{(2 \pi)^{1 / 2}} \int_{-\infty}^{+\infty} A_{l}\left(m-m_{1}, \epsilon\right) R_{l}\left(i m_{1}\right) \omega_{d_{1}}\left(s_{1}^{1 / k_{1}}, \tau_{2}, m_{1}, \epsilon\right) d m_{1}\right) \frac{d s_{1}}{s_{1}}+\sum_{1 \leq p \leq q-1} A_{q, p} \frac{\tau_{1}^{k_{1}}}{\Gamma\left(\frac{d_{l, q}+k_{1}(q-p)}{k_{1}}\right)} \\
\times \int_{0}^{\tau_{1}^{k_{1}}}\left(\tau_{1}^{k_{1}}-s_{1}\right)^{\frac{d_{l, q}+k_{1}(q-p)}{k_{1}}-1}(-1)^{p_{2}} \tau_{2}^{p_{2}}\left(k_{1}\left(s_{1}^{1 / k_{1}}\right)^{k_{1}}\right)^{p} \\
\left.\left.\left.\times\left(\frac{1}{(2 \pi)^{1 / 2}} \int_{-\infty}^{+\infty} A_{l}\left(m-m_{1}, \epsilon\right) R_{l}\left(i m_{1}\right) \omega_{d_{1}}\left(s_{1}^{1 / k_{1}}, \tau_{2}, m_{1}, \epsilon\right) d m_{1}\right) \frac{d s_{1}}{s_{1}}\right]\right)\right]+\mathcal{F}\left(\tau_{1}, \tau_{2}, m, \epsilon\right)
\end{gathered}
$$

\subsection{Action of linear convolution operators}

In this subsection, we investigate continuity properties of two useful linear convolutions operators acting on the Banach spaces given in Definition 4 and appearing in the above equation (42).

Proposition 1 Let $\gamma_{1} \geq 0, \gamma_{3} \geq-1$ be integers and set $\gamma_{2} \in \mathbb{R}$. Let $S_{d_{1}}$ be an unbounded sector 
centered at 0 with bisecting direction $d_{1} \in \mathbb{R}$ and set $\rho>0$ a positive real number.

Let $a_{\gamma_{1}}\left(\tau_{1}, m\right)$ be a continuous map on the closure $\left(\overline{S_{d_{1}}} \cup \overline{D_{\rho}}\right) \times \mathbb{R}$ subjected to the upper bounds

$$
\left|a_{\gamma_{1}}\left(\tau_{1}, m\right)\right| \leq \frac{M_{\gamma_{1}}}{\left(1+\left|\tau_{1}\right|\right)^{\gamma_{1}}}
$$

for all $\tau_{1} \in S_{d_{1}} \cup D_{\rho}$, all $m \in \mathbb{R}$, for some constant $M_{\gamma_{1}}>0$. Assume that

$$
\gamma_{1} \geq k_{1}\left(\gamma_{3}+1\right) \quad, \quad \gamma_{2}>-1, \quad \gamma_{2}+\gamma_{3}+\frac{1}{k_{1}}+1 \geq 0
$$

Then, a constant $C_{1}>0$ (relying on $\gamma_{1}, \gamma_{2}, \gamma_{3}, k_{1}$ and $\nu$ ) can found with

$$
\begin{aligned}
\| a_{\gamma_{1}}\left(\tau_{1}, m\right) \tau_{1}^{k_{1}} \int_{0}^{\tau_{1}^{k_{1}}}\left(\tau_{1}^{k_{1}}-s_{1}\right)^{\gamma_{2}} s_{1}^{\gamma_{3}} f\left(s_{1}^{1 / k_{1}}, \tau_{2}, m\right) d s_{1}||_{\left(\nu, \beta, \mu, k_{1}, \rho, a, \epsilon\right)} \\
\quad \leq C_{1} M_{\gamma_{1}}|\epsilon|^{k_{1}\left(\gamma_{2}+1\right)}\left\|f\left(\tau_{1}, \tau_{2}, m\right)\right\|_{\left(\nu, \beta, \mu, k_{1}, \rho, a, \epsilon\right)}
\end{aligned}
$$

for all $f \in F_{\left(\nu, \beta, \mu, k_{1}, \rho, a, \epsilon\right)}^{d_{1}}$.

Proof Let $f$ be an element of $F_{\left(\nu, \beta, \mu, k_{1}, \rho, a, \epsilon\right)}^{d_{1}}$. By definition, the next bounds

$$
\left|f\left(\tau_{1}, \tau_{2}, m\right)\right| \leq\left.|| f\right|_{\left(\nu, \beta, \mu, k_{1}, \rho, a, \epsilon\right)}\left|\frac{\tau_{1}}{\epsilon}\right| \exp \left(\nu\left|\frac{\tau_{1}}{\epsilon}\right|^{k_{1}}\right)\left|\tau_{2}\right|(1+|m|)^{-\mu} e^{-\beta|m|}
$$

hold whenever $\tau_{1} \in S_{d_{1}} \cup D_{\rho}, \tau_{2} \in D_{a}$, all $m \in \mathbb{R}$. These latter bounds together with the assumption (43) enable the next estimates

$$
\begin{aligned}
& \mathcal{A}\left(\tau_{1}, \tau_{2}, m\right) \\
& \quad:=\left|a_{\gamma_{1}}\left(\tau_{1}, m\right) \tau_{1}^{k_{1}} \int_{0}^{\tau_{1}^{k_{1}}}\left(\tau_{1}^{k_{1}}-s_{1}\right)^{\gamma_{2}} s_{1}^{\gamma_{3}} f\left(s_{1}^{1 / k_{1}}, \tau_{2}, m\right) d s_{1}\right| \leq \frac{M_{\gamma_{1}}|| f \|_{\left(\nu, \beta, \mu, k_{1}, \rho, a, \epsilon\right)}}{\left(1+\left|\tau_{1}\right|\right)^{\gamma_{1}}} \\
& \quad \times\left|\tau_{1}\right|^{k_{1}} \int_{0}^{\left|\tau_{1}\right|^{k_{1}}}\left(\left|\tau_{1}\right|^{k_{1}}-h_{1}\right)^{\gamma_{2}} h_{1}^{\gamma_{3}} \frac{h_{1}^{1 / k_{1}}}{|\epsilon|} \exp \left(\nu \frac{h_{1}}{|\epsilon|^{k_{1}}}\right) d h_{1}\left|\tau_{2}\right|(1+|m|)^{-\mu} e^{-\beta|m|}
\end{aligned}
$$

provided that $\tau_{1} \in S_{d_{1}} \cup D_{\rho}, \tau_{2} \in D_{a}$ and $m \in \mathbb{R}$. We further make the change of variable $g_{1}=h_{1} /|\epsilon|^{k_{1}}$ inside the integral appearing in the righthandside of the above inequality and get

$$
\begin{aligned}
\mathcal{A}\left(\tau_{1}, \tau_{2}, m\right) \leq & \frac{M_{\gamma_{1}}|| f \|_{\left(\nu, \beta, \mu, k_{1}, \rho, a, \epsilon\right)}}{\left(1+\left|\tau_{1}\right|\right)^{\gamma_{1}}}\left|\tau_{1}\right|^{k_{1}} \\
& \times \int_{0}^{\frac{\left|\tau_{1}\right|_{1}^{k_{1}}}{|\epsilon|^{k_{1}}}}\left(\frac{\left|\tau_{1}\right|^{k_{1}}}{|\epsilon|^{k_{1}}}-g_{1}\right)^{\gamma_{2}} g_{1}^{\gamma_{3}+\frac{1}{k_{1}}} e^{\nu g_{1}} d g_{1}|\epsilon|^{k_{1}\left(\gamma_{2}+\gamma_{3}+1\right)}\left|\tau_{2}\right|(1+|m|)^{-\mu} e^{-\beta|m|}
\end{aligned}
$$

as long as $\tau_{1} \in S_{d_{1}} \cup D_{\rho}, \tau_{2} \in D_{a}$ and $m \in \mathbb{R}$.

We consider the map

$$
G(x)=\int_{0}^{x}\left(x-g_{1}\right)^{\gamma_{2}} g_{1}^{\gamma_{3}+\frac{1}{k_{1}}} e^{\nu g_{1}} d g_{1}
$$

for all $x \geq 0$. Notice that $G(x)$ is well defined for all $x \geq 0$, according to (44), since we can recast its expression by means of the change of variable $g_{1}=x g_{2}$ for $0 \leq g_{2} \leq 1$ in the form

$$
G(x)=x^{\gamma_{2}+\gamma_{3}+\frac{1}{k_{1}}+1} \int_{0}^{1}\left(1-g_{2}\right)^{\gamma_{2}} g_{2}^{\gamma_{3}+\frac{1}{k_{1}}} e^{\nu x g_{2}} d g_{2}
$$


for all $x \geq 0$. According to the sharp bounds reached in Proposition 1 of the paper [10], we can single out a constant $K_{1}>0$ (depending on the constants $\gamma_{2}, \gamma_{3}, k_{1}, \nu$ ) for which

$$
G(x) \leq K_{1} x^{\gamma_{3}+\frac{1}{k_{1}}} e^{\nu x}
$$

for all $x \geq 1$.

Two cases arise.

A) Assume first that $\tau_{1} \in S_{d_{1}} \cup D_{\rho}$ is chosen such that

$$
\frac{\left|\tau_{1}\right|^{k_{1}}}{|\epsilon|^{k_{1}}}>1
$$

Then, under the condition (44), one finds a constant $C_{1.1}>0$ (relying on $\gamma_{1}, \gamma_{3}, k_{1}, K_{1}$ ) such that

$$
\begin{aligned}
\mathcal{A}\left(\tau_{1}, \tau_{2}, m\right) \leq & \frac{M_{\gamma_{1}}|| f \|_{\left(\nu, \beta, \mu, k_{1}, \rho, a, \epsilon\right)}}{\left(1+\left|\tau_{1}\right|\right)^{\gamma_{1}}}\left|\tau_{1}\right|^{k_{1}}|\epsilon|^{k_{1}\left(\gamma_{2}+\gamma_{3}+1\right)} K_{1}\left(\frac{\left|\tau_{1}\right|}{|\epsilon|}\right)^{k_{1}\left(\gamma_{3}+\frac{1}{k_{1}}\right)} \\
\times \exp \left(\nu \frac{\left|\tau_{1}\right|^{k_{1}}}{|\epsilon|^{k_{1}}}\right)\left|\tau_{2}\right|(1+|m|)^{-\mu} e^{-\beta|m|} \leq & C_{1.1} M_{\gamma_{1}}|\epsilon|^{k_{1}\left(\gamma_{2}+1\right)}|| f \|_{\left(\nu, \beta, \mu, k_{1}, \rho, a, \epsilon\right)} \\
& \times\left|\frac{\tau_{1}}{\epsilon}\right| \exp \left(\nu\left|\frac{\tau_{1}}{\epsilon}\right|^{k_{1}}\right)\left|\tau_{2}\right|(1+|m|)^{-\mu} e^{-\beta|m|}
\end{aligned}
$$

for all $\tau_{1} \in S_{d_{1}} \cup D_{\rho}$, under the constraint (50), all $\tau_{2} \in D_{a}$ and $m \in \mathbb{R}$.

B) Take for granted that $\tau_{1} \in S_{d_{1}} \cup D_{\rho}$ fufills

$$
0 \leq \frac{\left|\tau_{1}\right|^{k_{1}}}{|\epsilon|^{k_{1}}} \leq 1
$$

Then, owing to (48), there exists a constant $C_{1.2}>0$ (leaning on $\left.\gamma_{2}, \gamma_{3}, k_{1}\right)$ with

$$
\begin{aligned}
& \mathcal{A}\left(\tau_{1}, \tau_{2}, m\right) \leq \frac{M_{\gamma_{1}}|| f \|_{\left(\nu, \beta, \mu, k_{1}, \rho, a, \epsilon\right)}}{\left(1+\left|\tau_{1}\right|\right)^{\gamma_{1}}}\left|\tau_{1}\right|^{k_{1}} \int_{0}^{\frac{\left|\tau_{1}\right|^{k_{1}}}{|\epsilon|^{k_{1}}}}\left(\frac{\left|\tau_{1}\right|^{k_{1}}}{|\epsilon|^{k_{1}}}-g_{1}\right)^{\gamma_{2}} g_{1}^{\gamma_{3}+\frac{1}{k_{1}}} d g_{1} \\
& \times \exp \left(\nu \frac{\left|\tau_{1}\right|^{k_{1}}}{|\epsilon|^{k_{1}}}\right)|\epsilon|^{k_{1}\left(\gamma_{2}+\gamma_{3}+1\right)}\left|\tau_{2}\right|(1+|m|)^{-\mu} e^{-\beta|m|} \leq\left.|| f||\right|_{\left(\nu, \beta, \mu, k_{1}, \rho, a, \epsilon\right)}\left|\frac{\tau_{1}}{\epsilon}\right| \exp \left(\nu \frac{\left|\tau_{1}\right|^{k_{1}}}{|\epsilon|^{k_{1}}}\right)\left|\tau_{2}\right| \\
& \times(1+|m|)^{-\mu} e^{-\beta|m|} \times\left[C_{1.2} M_{\gamma_{1}}\left|\tau_{1}\right|^{k_{1}-1}|\epsilon|^{k_{1}\left(\gamma_{2}+1\right)}|\epsilon|^{1+k_{1} \gamma_{3}}\right] \\
& \leq C_{1.2} M_{\gamma_{1}} \epsilon_{0}^{k_{1}\left(\gamma_{3}+1\right)}|\epsilon|^{k_{1}\left(\gamma_{2}+1\right)}|| f \|_{\left(\nu, \beta, \mu, k_{1}, \rho, a, \epsilon\right)} \times\left|\frac{\tau_{1}}{\epsilon}\right| \exp \left(\nu\left|\frac{\tau_{1}}{\epsilon}\right|^{k_{1}}\right)\left|\tau_{2}\right|(1+|m|)^{-\mu} e^{-\beta|m|}
\end{aligned}
$$

for all $\tau_{1} \in S_{d_{1}} \cup D_{\rho}$ submitted to (52), all $\tau_{2} \in D_{a}$ and $m \in \mathbb{R}$.

At last, the collection of the upper bound (47) coupled with (51) and (53) spawns the awaited estimates (45).

Proposition 2 Let $Q(X), R(X) \in \mathbb{C}[X]$ be polynomials submitted to the constraints

$$
\operatorname{deg}(R) \geq \operatorname{deg}(Q) \quad, \quad R(i m) \neq 0 \quad, \quad \mu>\operatorname{deg}(Q)+1
$$

for all $m \in \mathbb{R}$. Then, one can select a constant $C_{2}>0$ (depending on $Q, R, \mu$ ) such that

$$
\begin{aligned}
\| \frac{1}{R(i m)} \int_{-\infty}^{+\infty} f\left(m-m_{1}\right) Q\left(i m_{1}\right) g\left(\tau_{1}, \tau_{2}, m_{1}\right) & d m_{1} \|_{\left(\nu, \beta, \mu, k_{1}, \rho, a, \epsilon\right)} \\
& \leq C_{2}\|f(m)\|_{(\beta, \mu)}\left\|g\left(\tau_{1}, \tau_{2}, m\right)\right\|_{\left(\nu, \beta, \mu, k_{1}, \rho, a, \epsilon\right)}
\end{aligned}
$$

whenever $f \in E_{(\beta, \mu)}$ and $g \in F_{\left(\nu, \beta, \mu, k_{1}, \rho, a, \epsilon\right)}^{d_{1}}$. 
Proof We take $g$ in $F_{\left(\nu, \beta, \mu, k_{1}, \rho, a, \epsilon\right)}^{d_{1}}$. From the very definition of the norms displayed in Definitions 2 and 4 , we arrive at

$$
\left|g\left(\tau_{1}, \tau_{2}, m_{1}\right)\right| \leq\left.|| g\right|_{\left(\nu, \beta, \mu, k_{1}, \rho, a, \epsilon\right)}\left|\frac{\tau_{1}}{\epsilon}\right| \exp \left(\nu\left|\frac{\tau_{1}}{\epsilon}\right|^{k_{1}}\right)\left|\tau_{2}\right|\left(1+\left|m_{1}\right|\right)^{-\mu} e^{-\beta\left|m_{1}\right|}
$$

provided that $\tau_{1} \in S_{d_{1}} \cup D_{\rho}, \tau_{2} \in D_{a}$ and $m_{1} \in \mathbb{R}$ together with

$$
|f(m)| \leq\|f(m)\|_{(\beta, \mu)}(1+|m|)^{-\mu} e^{-\beta|m|}
$$

for all $m \in \mathbb{R}$. These two bounds (56) and (57) allow the next estimates

$$
\begin{aligned}
& \left|\mathcal{B}\left(\tau_{1}, \tau_{2}, m\right)\right|:=\left|\frac{1}{R(i m)} \int_{-\infty}^{+\infty} f\left(m-m_{1}\right) Q\left(i m_{1}\right) g\left(\tau_{1}, \tau_{2}, m_{1}\right) d m_{1}\right| \\
& \quad \leq|| f(m) \|\left._{(\beta, \mu)}|| g\left(\tau_{1}, \tau_{2}, m\right)\right|_{\left(\nu, \beta, \mu, k_{1}, \rho, a, \epsilon\right)}\left|\frac{\tau_{1}}{\epsilon}\right| \exp \left(\left.\nu\left|\frac{\tau_{1}}{\epsilon}\right|\right|^{k_{1}}\right)\left|\tau_{2}\right|(1+|m|)^{-\mu} e^{-\beta|m|} C_{2.1}
\end{aligned}
$$

where

$$
C_{2.1}=(1+|m|)^{\mu} e^{\beta|m|} \frac{1}{|R(i m)|} \int_{-\infty}^{+\infty} \frac{e^{-\beta\left|m-m_{1}\right|}}{\left(1+\left|m-m_{1}\right|\right)^{\mu}} \frac{\left|Q\left(i m_{1}\right)\right|}{\left(1+\left|m_{1}\right|\right)^{\mu}} e^{-\beta\left|m_{1}\right|} d m_{1} .
$$

According to the triangular inequality, we know that $|m| \leq\left|m-m_{1}\right|+\left|m_{1}\right|$ for all real numbers $m, m_{1} \in \mathbb{R}$ and by construction of the polynomials $R, Q$ asked to fulfill (54), two constants $\mathfrak{Q}, \mathfrak{R}>0$ can be singled out such that

$$
\left|Q\left(i m_{1}\right)\right| \leq \mathfrak{Q}\left(1+\left|m_{1}\right|\right)^{\operatorname{deg}(Q)} \quad, \quad|R(i m)| \geq \mathfrak{R}(1+|m|)^{\operatorname{deg}(R)}
$$

whenever $m, m_{1} \in \mathbb{R}$. Thereby, we come to the upper bounds

$$
C_{2.1} \leq \frac{\mathfrak{Q}}{\mathfrak{R}} \sup _{m \in \mathbb{R}}(1+|m|)^{\mu-\operatorname{deg}(R)} \int_{-\infty}^{+\infty} \frac{1}{\left(1+\left|m-m_{1}\right|\right)^{\mu}\left(1+\left|m_{1}\right|\right)^{\mu-\operatorname{deg}(Q)}} d m_{1}
$$

whose right handside is a finite quantity under the restrictions (54), owing to Lemma 2.2 from [5] or Lemma 4 of [14].

Eventually, gathering (58) and (59) yields the foretold bounds (55).

\subsection{Solving the associated convolution equation}

In this subsection we uniquely solve the auxiliary convolution equation reached in (42) within the Banach spaces displayed in Definition 4 by means of the properties of the convolution operators obtained in Subsection 3.2.

Our strategy consists in recasting (42) into a fixed point equation (stated later on in (91)). In the process, we need to perform a division by the next parameter depending polynomial

$$
P_{m}\left(\tau_{1}\right):=Q(i m)-R_{D}(i m) k_{1}^{\delta_{D}} \tau_{1}^{k_{1} \delta_{D}}
$$

provided that $\tau_{1} \in S_{d_{1}} \cup D_{\rho}$. Crucial lower bounds are stated in the next lemma.

Lemma 4 For a suitable choice of the inner radius $r_{Q, R_{D}}>0$ and aperture $\eta_{Q, R_{D}}>0$ of the sector $S_{Q, R_{D}}$ set up in (15), there exist unbounded sectors $S_{d_{1}}$ centered at 0 with bisecting direction $d_{1} \in \mathbb{R}$ and a small enough radius $\rho>0$ for which the next lower estimates hold. One can select a constant $C_{P}>0$ with

$$
\left|P_{m}\left(\tau_{1}\right)\right| \geq C_{P}\left(r_{Q, R_{D}}\right)^{\frac{1}{k_{1} \delta_{D}}}\left|R_{D}(i m)\right|\left(1+\left|\tau_{1}\right|\right)^{k_{1} \delta_{D}-1}
$$

provided that $\tau_{1} \in S_{d_{1}} \cup D_{\rho}$, for all $m \in \mathbb{R}$. 
Proof Since the complex roots $q_{l}(m), 0 \leq l \leq k_{1} \delta_{D}-1$ of $\tau_{1} \mapsto P_{m}\left(\tau_{1}\right)$ are explicit, we can factorize the polynomial as follows

$$
P_{m}\left(\tau_{1}\right)=-R_{D}(i m) k_{1}^{\delta_{D}} \Pi_{l=0}^{k_{1} \delta_{D}-1}\left(\tau_{1}-q_{l}(m)\right)
$$

where

$$
q_{l}(m)=\left(\frac{|Q(i m)|}{\left|R_{D}(i m)\right| k_{1}^{\delta_{D}}}\right)^{\frac{1}{k_{1} \delta_{D}}} \exp \left(\sqrt{-1}\left(\arg \left(\frac{Q(i m)}{R_{D}(i m) k_{1}^{\delta_{D}}}\right) \frac{1}{k_{1} \delta_{D}}+\frac{2 \pi l}{k_{1} \delta_{D}}\right)\right)
$$

for all $0 \leq l \leq k_{1} \delta_{D}-1$, for any $\tau_{1} \in \mathbb{C}$ and $m \in \mathbb{R}$.

We single out an unbounded sector $S_{d_{1}}$ centered at 0 , a small disc $D_{\rho}$ and we arrange the sector $S_{Q, R_{D}}$ given in (15) in a way that the next two features hold:

1) A constant $M_{1}>0$ can be found such that

$$
\left|\tau_{1}-q_{l}(m)\right| \geq M_{1}\left(1+\left|\tau_{1}\right|\right)
$$

for all $0 \leq l \leq k_{1} \delta_{D}-1$, all $m \in \mathbb{R}$, whenever $\tau_{1} \in S_{d_{1}} \cup D_{\rho}$.

2) There exists a constant $M_{2}>0$ with

$$
\left|\tau_{1}-q_{l_{0}}(m)\right| \geq M_{2}\left|q_{l_{0}}(m)\right|
$$

for some $0 \leq l_{0} \leq \delta_{D} k_{1}-1$, all $m \in \mathbb{R}$, all $\tau_{1} \in S_{d_{1}} \cup D_{\rho}$.

We now provide some explanations for the two above bounds.

- Concerning the first point 1$)$, we observe that under the hypothesis $(15)$, the roots $q_{l}(m)$ are bounded from below and satisfy $\left|q_{l}(m)\right| \geq 2 \rho$ for all $m \in \mathbb{R}$, all $0 \leq l \leq \delta_{D} k_{1}-1$ for a suitable choice of the radii $r_{Q, R_{D}}, \rho>0$. Furthermore, for all $m \in \mathbb{R}$, all $0 \leq l \leq \delta_{D} k_{1}-1$, these roots remain inside an union $\mathcal{Q}$ of unbounded sectors centered at 0 that do not cover a full neighborhood of 0 in $\mathbb{C}^{*}$ whenever the aperture $\eta_{Q, R_{D}}>0$ of $S_{Q, R_{D}}$ is taken small enough. Therefore, we may choose a sector $S_{d_{1}}$ such that

$$
S_{d_{1}} \cap \mathcal{Q}=\emptyset .
$$

Such sector satisfies that for all $0 \leq l \leq \delta_{D} k_{1}-1$, the quotients $q_{l}(m) / \tau_{1}$ lay outside some small disc centered at 1 in $\mathbb{C}$ for all $\tau_{1} \in S_{d_{1}}$, all $m \in \mathbb{R}$. As a consequence, (63) follows.

- We select the sector $S_{d_{1}}$ and disc $D_{\rho}$ as above. The second point 2) follows from the fact that for any fixed $0 \leq l_{0} \leq \delta_{D} k_{1}-1$, the quotient $\tau_{1} / q_{l_{0}}(m)$ stays apart a small disc centered at 1 in $\mathbb{C}$ for all $\tau_{1} \in S_{d_{1}} \cup D_{\rho}$, all $m \in \mathbb{R}$.

Departing from the factorization (62) and taking benefit from the two lower bounds (63), (64) reached overhead, we arrive at

$$
\begin{aligned}
\left|P_{m}\left(\tau_{1}\right)\right| \geq M_{1}^{k_{1} \delta_{D}-1} M_{2}\left|R_{D}(i m)\right| k_{1}^{\delta_{D}}\left(\frac{|Q(i m)|}{\mid R_{D}(i m) k_{1}^{\delta_{D}}}\right)^{\frac{1}{k_{1} \delta_{D}}}\left(1+\left|\tau_{1}\right|\right)^{k_{1} \delta_{D}-1} & \\
& \geq C_{P}\left(r_{Q, R_{D}}\right)^{\frac{1}{k_{1} \delta_{D}}}\left|R_{D}(i m)\right|\left(1+\left|\tau_{1}\right|\right)^{k_{1} \delta_{D}-1}
\end{aligned}
$$

as long as $\tau_{1} \in S_{d_{1}} \cup D_{\rho}$, for all $m \in \mathbb{R}$. 
We introduce the next linear map

(66)

$$
\begin{aligned}
& :=\frac{\tau_{1}^{k_{1}}}{P_{m}\left(\tau_{1}\right) \Gamma\left(\frac{d_{D}}{k_{1}}\right)} \int_{0}^{\tau_{1}^{k_{1}}}\left(\tau_{1}^{k_{1}}-s_{1}\right)^{\frac{d_{D}}{k_{1}}-1} \tau_{2}^{\delta_{D}}(-1)^{\delta_{D}} R_{D}(i m) \omega\left(s_{1}^{1 / k_{1}}, \tau_{2}, m\right) \frac{d s_{1}}{s_{1}} \\
& +\sum_{\substack{p_{1}+p_{2}=\delta_{D} \\
1 \leq p_{1}<\delta_{D}}} \frac{\delta_{D} !}{p_{1} ! p_{2} !}\left(\sum _ { q = 1 } ^ { p _ { 1 } } a _ { q , p _ { 1 } } \left[\frac{\tau_{1}^{k_{1}}}{P_{m}\left(\tau_{1}\right) \Gamma\left(\frac{d_{D, q}}{k_{1}}\right)} \int_{0}^{\tau_{1}^{k_{1}}}\left(\tau_{1}^{k_{1}}-s_{1}\right)^{\frac{d_{D, q}}{k_{1}}-1} k_{1}^{q} s_{1}^{q} \omega\left(s_{1}^{1 / k_{1}}, \tau_{2}, m\right) \frac{d s_{1}}{s_{1}}\right.\right. \\
& \left.\left.+\sum_{1 \leq p \leq q-1} A_{q, p} \frac{\tau_{1}^{k_{1}}}{P_{m}\left(\tau_{1}\right) \Gamma\left(\frac{d_{D, q}+k_{1}(q-p)}{k_{1}}\right)} \int_{0}^{\tau_{1}^{k_{1}}}\left(\tau_{1}^{k_{1}}-s_{1}\right)^{\frac{d_{D, q}+k_{1}(q-p)}{k_{1}}-1} k_{1}^{p} s_{1}^{p} \omega\left(s_{1}^{1 / k_{1}}, \tau_{2}, m\right) \frac{d s_{1}}{s_{1}}\right]\right) \\
& \times(-1)^{p_{2}} \tau_{2}^{p_{2}} R_{D}(i m)+\sum_{q=1}^{\delta_{D}-1} a_{q, \delta_{D}}\left[\frac{\tau_{1}^{k_{1}}}{P_{m}\left(\tau_{1}\right) \Gamma\left(\frac{d_{D, q}}{k_{1}}\right)} \int_{0}^{\tau_{1}^{k_{1}}}\left(\tau_{1}^{k_{1}}-s_{1}\right)^{\frac{d_{D, q}}{k_{1}}-1} k_{1}^{q} s_{1}^{q} \omega\left(s_{1}^{1 / k_{1}}, \tau_{2}, m\right) \frac{d s_{1}}{s_{1}}\right. \\
& \left.+\sum_{1 \leq p \leq q-1} A_{q, p} \frac{\tau_{1}^{k_{1}}}{P_{m}\left(\tau_{1}\right) \Gamma\left(\frac{d_{D, q}+k_{1}(q-p)}{k_{1}}\right)} \int_{0}^{\tau_{1}^{k_{1}}}\left(\tau_{1}^{k_{1}}-s_{1}\right)^{\frac{d_{D, q}+k_{1}(q-p)}{k_{1}}-1} k_{1}^{p} s_{1}^{p} \omega\left(s_{1}^{1 / k_{1}}, \tau_{2}, m\right) \frac{d s_{1}}{s_{1}}\right] \\
& \times R_{D}(i m) \\
& +\sum_{1 \leq p \leq \delta_{D}-1} A_{\delta_{D}, p} \frac{\tau_{1}^{k_{1}}}{P_{m}\left(\tau_{1}\right) \Gamma\left(\delta_{D}-p\right)} \int_{0}^{\tau_{1}^{k_{1}}}\left(\tau_{1}^{k_{1}}-s_{1}\right)^{\delta_{D}-p-1} k_{1}^{p} s_{1}^{p} \omega\left(s_{1}^{1 / k_{1}}, \tau_{2}, m\right) \frac{d s_{1}}{s_{1}} R_{D}(i m) \\
& +\sum_{l=1}^{D-1} \epsilon^{\Delta_{l}-d_{l}}\left[\frac{\tau_{1}^{k_{1}}}{P_{m}\left(\tau_{1}\right) \Gamma\left(\frac{d_{l}}{k_{1}}\right)} \int_{0}^{\tau_{1}^{k_{1}}}\left(\tau_{1}^{k_{1}}-s_{1}\right)^{\frac{d_{l}}{k_{1}}-1} \tau_{2}^{\delta_{l}}(-1)^{\delta_{l}}\right. \\
& \times\left(\frac{1}{(2 \pi)^{1 / 2}} \int_{-\infty}^{+\infty} A_{l}\left(m-m_{1}, \epsilon\right) R_{l}\left(i m_{1}\right) \omega\left(s_{1}^{1 / k_{1}}, \tau_{2}, m_{1}\right) d m_{1}\right) \frac{d s_{1}}{s_{1}}+\sum_{\substack{p_{1}+p_{2}=\delta_{l} \\
1 \leq p_{1} \leq \delta_{l}}} \frac{\delta_{l} !}{p_{1} ! p_{2} !}\left(\sum_{q=1}^{p_{1}} a_{q, p_{1}}\right. \\
& \times\left[\frac{\tau_{1}^{k_{1}}}{P_{m}\left(\tau_{1}\right) \Gamma\left(\frac{d_{l, q}}{k_{1}}\right)} \int_{0}^{\tau_{1}^{k_{1}}}\left(\tau_{1}^{k_{1}}-s_{1}\right)^{\frac{d_{l, q}}{k_{1}}-1}(-1)^{p_{2}} \tau_{2}^{p_{2}} k_{1}^{q} s_{1}^{q}\right. \\
& \times\left(\frac{1}{(2 \pi)^{1 / 2}} \int_{-\infty}^{+\infty} A_{l}\left(m-m_{1}, \epsilon\right) R_{l}\left(i m_{1}\right) \omega\left(s_{1}^{1 / k_{1}}, \tau_{2}, m_{1}\right) d m_{1}\right) \frac{d s_{1}}{s_{1}}+\sum_{1 \leq p \leq q-1} A_{q, p} \frac{\tau_{1}^{k_{1}}}{P_{m}\left(\tau_{1}\right) \Gamma\left(\frac{d_{l, q}+k_{1}(q-p)}{k_{1}}\right)} \\
& \times \int_{0}^{\tau_{1}^{k_{1}}}\left(\tau_{1}^{k_{1}}-s_{1}\right)^{\frac{d_{l, q}+k_{1}(q-p)}{k_{1}}-1}(-1)^{p_{2}} \tau_{2}^{p_{2}} k_{1}^{p} s_{1}^{p} \\
& \left.\left.\left.\times\left(\frac{1}{(2 \pi)^{1 / 2}} \int_{-\infty}^{+\infty} A_{l}\left(m-m_{1}, \epsilon\right) R_{l}\left(i m_{1}\right) \omega\left(s_{1}^{1 / k_{1}}, \tau_{2}, m_{1}\right) d m_{1}\right) \frac{d s_{1}}{s_{1}}\right]\right)\right]+\frac{\mathcal{F}\left(\tau_{1}, \tau_{2}, m, \epsilon\right)}{P_{m}\left(\tau_{1}\right)}
\end{aligned}
$$

In the next proposition, we show that $\mathcal{H}_{\epsilon}$ represents a $1 / 2$-Lipschitz map on some suitable ball of the Banach space introduced in Definition 4.

Proposition 3 We select an appropriate inner radius $r_{Q, R_{D}}>0$ and aperture $\eta_{Q, R_{D}}>0$ of the sector $S_{Q, R_{D}}$ defined in (15), together with an unbounded sector $S_{d_{1}}$ and a radius $\rho$ that fulfill the requirements of Lemma 4. Then, one can choose a radius $\epsilon_{0}>0$ small enough and a fitting radius $\varpi>0$ in a way that for all $\epsilon \in D_{\epsilon_{0}} \backslash\{0\}$, the map $\mathcal{H}_{\epsilon}$ carries the next two features 
- The inclusion

$$
\mathcal{H}_{\epsilon}\left(\bar{B}_{\varpi}\right) \subset \bar{B}_{\varpi}
$$

holds, where $\bar{B}_{\varpi}$ stands for the closed ball of radius $\varpi>0$ centered at 0 in the space $F_{\left(\nu, \beta, \mu, k_{1}, \rho, a, \epsilon\right)}^{d_{1}}$.

- The shrinking condition

$$
\left\|\mathcal{H}_{\epsilon}\left(\omega_{1}\right)-\mathcal{H}_{\epsilon}\left(\omega_{2}\right)\right\|_{\left(\nu, \beta, \mu, k_{1}, \rho, a, \epsilon\right)} \leq \frac{1}{2}\left\|\omega_{1}-\omega_{2}\right\|_{\left(\nu, \beta, \mu, k_{1}, \rho, a, \epsilon\right)}
$$

occurs whenever $\omega_{1}, \omega_{2} \in \bar{B}_{\varpi}$.

Proof We focus on the first item heralding the inclusion (67). We fix some real number $\varpi>0$ and we take some $\omega\left(\tau_{1}, \tau_{2}, m\right)$ that belongs to $F_{\left(\nu, \beta, \mu, k_{1}, \rho, a, \epsilon\right)}^{d_{1}}$, for $\epsilon \in D_{\epsilon_{0}} \backslash\{0\}$, such that

$$
\|\omega\|_{\left(\nu, \beta, \mu, k_{1}, \rho, a, \epsilon\right)} \leq \varpi
$$

In the sequel, we provide bounds estimates for each piece of the map $\mathcal{H}_{\epsilon}$.

In the next six estimates, we make use of the bounds obtained in Proposition 1 and Lemma 4. Namely, owing to the equality in (12) we get

$$
\begin{aligned}
\left\|\frac{\tau_{1}^{k_{1}}}{P_{m}\left(\tau_{1}\right)} \int_{0}^{\tau_{1}^{k_{1}}}\left(\tau_{1}^{k_{1}}-s_{1}\right)^{\frac{d_{D}}{k_{1}}-1} \tau_{2}^{\delta_{D}}(-1)^{\delta_{D}} R_{D}(i m) \omega\left(s_{1}^{1 / k_{1}}, \tau_{2}, m\right) \frac{d s_{1}}{s_{1}}\right\|_{\left(\nu, \beta, \mu, k_{1}, \rho, a, \epsilon\right)} \\
\leq \frac{C_{1}}{C_{P}\left(r_{Q, R_{D}}\right)^{\frac{1}{k_{1} \delta_{D}}}} a^{\delta_{D}}|\epsilon|^{d_{D}}\left\|\omega\left(\tau_{1}, \tau_{2}, m\right)\right\|_{\left(\nu, \beta, \mu, k_{1}, \rho, a, \epsilon\right)} \\
\quad \leq \frac{C_{1}}{C_{P}\left(r_{Q, R_{D}}\right)^{\frac{1}{k_{1} \delta_{D}}}} a^{\delta_{D}} \epsilon_{0}^{d_{D}} \varpi .
\end{aligned}
$$

Besides, we check that

$$
\begin{array}{r}
\left\|\frac{\tau_{1}^{k_{1}}}{P_{m}\left(\tau_{1}\right)} \int_{0}^{\tau_{1}^{k_{1}}}\left(\tau_{1}^{k_{1}}-s_{1}\right)^{\frac{d_{D, q}}{k_{1}}-1}(-1)^{p_{2}} \tau_{2}^{p_{2}} R_{D}(i m) s_{1}^{q-1} \omega\left(s_{1}^{1 / k_{1}}, \tau_{2}, m\right) d s_{1}\right\|_{\left(\nu, \beta, \mu, k_{1}, \rho, a, \epsilon\right)} \\
\leq \frac{C_{1}}{C_{P}\left(r_{Q, R_{D}}\right)^{\frac{1}{k_{1} \delta_{D}}}} a^{p_{2}}|\epsilon|^{d_{D, q}}\left\|\omega\left(\tau_{1}, \tau_{2}, m\right)\right\| \|_{\left(\nu, \beta, \mu, k_{1}, \rho, a, \epsilon\right)} \\
\quad \leq \frac{C_{1}}{C_{P}\left(r_{Q, R_{D}}\right)^{\frac{1}{k_{1} \delta_{D}}}} a^{p_{2}} \epsilon_{0}^{d_{D, q}} \varpi
\end{array}
$$

holds for $1 \leq q \leq \delta_{D}-1$, along with

$$
\begin{aligned}
& \| \frac{\tau_{1}^{k_{1}}}{P_{m}\left(\tau_{1}\right)} \int_{0}^{\tau_{1}^{k_{1}}}\left(\tau_{1}^{k_{1}}-s_{1}\right)^{\frac{d_{D, q}+k_{1}(q-p)}{k_{1}}-1}(-1)^{p_{2}} \tau_{2}^{p_{2}} \\
& \times R_{D}(i m) s_{1}^{p-1} \omega\left(s_{1}^{1 / k_{1}}, \tau_{2}, m\right) d s_{1} \|_{\left(\nu, \beta, \mu, k_{1}, \rho, a, \epsilon\right)} \leq \frac{C_{1}}{C_{P}\left(r_{Q, R_{D}}\right)^{\frac{1}{k_{1} \delta_{D}}}} a^{p_{2}}|\epsilon|^{d_{D, q}+k_{1}(q-p)} \\
& \quad \times\left\|\omega\left(\tau_{1}, \tau_{2}, m\right)\right\|_{\left(\nu, \beta, \mu, k_{1}, \rho, a, \epsilon\right)} \leq \frac{C_{1}}{C_{P}\left(r_{Q, R_{D}}\right)^{\frac{1}{k_{1} \delta_{D}}}} a^{p_{2}} \epsilon_{0}^{d_{D, q}+k_{1}(q-p)} \varpi
\end{aligned}
$$


for all $1 \leq p \leq q-1$ and $1 \leq q \leq \delta_{D}-1$.

Furthermore, we observe that

$$
\begin{aligned}
\left\|\frac{\tau_{1}^{k_{1}}}{P_{m}\left(\tau_{1}\right)} \int_{0}^{\tau_{1}^{k_{1}}}\left(\tau_{1}^{k_{1}}-s_{1}\right)^{\frac{d_{D, q}}{k_{1}}-1} s_{1}^{q-1} R_{D}(i m) \omega\left(s_{1}^{1 / k_{1}}, \tau_{2}, m\right) d s_{1}\right\|_{\left(\nu, \beta, \mu, k_{1}, \rho, a, \epsilon\right)} \\
\quad \leq \frac{C_{1}}{C_{P}\left(r_{Q, R_{D}}\right)^{\frac{1}{k_{1} \delta_{D}}}}|\epsilon|^{d_{D, q}}\left\|\omega\left(\tau_{1}, \tau_{2}, m\right)\right\|_{\left(\nu, \beta, \mu, k_{1}, \rho, a, \epsilon\right)} \leq \frac{C_{1}}{C_{P}\left(r_{Q, R_{D}}\right)^{\frac{1}{k_{1} \delta_{D}}}} \epsilon_{0}^{d_{D, q}} \varpi
\end{aligned}
$$

provided that $1 \leq q \leq \delta_{D}-1$, together with

$$
\begin{aligned}
& \left\|\frac{\tau_{1}^{k_{1}}}{P_{m}\left(\tau_{1}\right)} \int_{0}^{\tau_{1}^{k_{1}}}\left(\tau_{1}^{k_{1}}-s_{1}\right)^{\frac{d_{D, q}+k_{1}(q-p)}{k_{1}}-1} s_{1}^{p-1} R_{D}(i m) \omega\left(s_{1}^{1 / k_{1}}, \tau_{2}, m\right) d s_{1}\right\|_{\left(\nu, \beta, \mu, k_{1}, \rho, a, \epsilon\right)} \\
\leq & \frac{C_{1}}{C_{P}\left(r_{Q, R_{D}}\right)^{\frac{1}{k_{1} \delta_{D}}}}|\epsilon|^{d_{D, q}+k_{1}(q-p)}\left\|\omega\left(\tau_{1}, \tau_{2}, m\right)\right\|_{\left(\nu, \beta, \mu, k_{1}, \rho, a, \epsilon\right)} \leq \frac{C_{1}}{C_{P}\left(r_{Q, R_{D}}\right)^{\frac{1}{k_{1} \delta_{D}}}} \epsilon_{0}^{d_{D, q}+k_{1}(q-p)} \varpi
\end{aligned}
$$

whenever $1 \leq p \leq q-1,1 \leq q \leq \delta_{D}-1$ and

$$
\begin{aligned}
& \left\|\frac{\tau_{1}^{k_{1}}}{P_{m}\left(\tau_{1}\right)} \int_{0}^{\tau_{1}^{k_{1}}}\left(\tau_{1}^{k_{1}}-s_{1}\right)^{\delta_{D}-p-1} R_{D}(i m) s_{1}^{p-1} \omega\left(s_{1}^{1 / k_{1}}, \tau_{2}, m\right) d s_{1}\right\|_{\left(\nu, \beta, \mu, k_{1}, \rho, a, \epsilon\right)} \\
& \quad \leq \frac{C_{1}}{C_{P}\left(r_{Q, R_{D}}\right)^{\frac{1}{k_{1} \delta_{D}}}}|\epsilon|^{k_{1}\left(\delta_{D}-p\right)}\left\|\omega\left(\tau_{1}, \tau_{2}, m\right)\right\|_{\left(\nu, \beta, \mu, k_{1}, \rho, a, \epsilon\right)} \leq \frac{C_{1}}{C_{P}\left(r_{Q, R_{D}}\right)^{\frac{1}{k_{1} \delta_{D}}}} \epsilon_{0}^{k_{1}\left(\delta_{D}-p\right)} \varpi
\end{aligned}
$$

as long as $1 \leq p \leq \delta_{D}-1$.

In the upcoming next three upper bounds, we apply both Propositions 1, 2 and Lemma 4. Indeed, on the basis of the inequality in (12) and the first inequality in (13) combined with (14), (16) and (17), we get

$$
\begin{aligned}
& \| \epsilon^{\Delta_{l}-d_{l}} \frac{\tau_{1}^{k_{1}}}{P_{m}\left(\tau_{1}\right)} \int_{0}^{\tau_{1}^{k_{1}}}\left(\tau_{1}^{k_{1}}-s_{1}\right)^{\frac{d_{l}}{k_{1}}-1} \tau_{2}^{\delta_{l}}(-1)^{\delta_{l}} s_{1}^{-1} \\
& \times\left(\frac{1}{(2 \pi)^{1 / 2}} \int_{-\infty}^{+\infty} A_{l}\left(m-m_{1}, \epsilon\right) R_{l}\left(i m_{1}\right) \omega\left(s_{1}^{1 / k_{1}}, \tau_{2}, m_{1}\right) d m_{1}\right) d s_{1} \|_{\left(\nu, \beta, \mu, k_{1}, \rho, a, \epsilon\right)} \\
& \quad=\| \epsilon^{\Delta_{l}-d_{l}} \frac{R_{D}(i m)}{P_{m}\left(\tau_{1}\right)} \times \tau_{1}^{k_{1}} \int_{0}^{\tau_{1}^{k_{1}}}\left(\tau_{1}^{k_{1}}-s_{1}\right)^{\frac{d_{l}}{k_{1}}-1} \tau_{2}^{\delta_{l}}(-1)^{\delta_{l}} s_{1}^{-1} \\
& \times\left(\frac{1}{(2 \pi)^{1 / 2} R_{D}(i m)} \int_{-\infty}^{+\infty} A_{l}\left(m-m_{1}, \epsilon\right) R_{l}\left(i m_{1}\right) \omega\left(s_{1}^{1 / k_{1}}, \tau_{2}, m_{1}\right) d m_{1}\right) d s_{1} \|_{\left(\nu, \beta, \mu, k_{1}, \rho, a, \epsilon\right)} \\
& \quad \leq \frac{C_{1}}{C_{P}\left(r_{Q, R_{D}}\right)^{\frac{1}{k_{1} \delta_{D}}}|\epsilon|^{\Delta_{l}-d_{l}}|\epsilon|^{d_{l}} a^{\delta_{l}}} \\
& \quad \times \frac{1}{(2 \pi)^{1 / 2} R_{D}(i m)} \int_{-\infty}^{+\infty} A_{l}\left(m-m_{1}, \epsilon\right) R_{l}\left(i m_{1}\right) \omega\left(\tau_{1}, \tau_{2}, m_{1}\right) d m_{1} \|_{\left(\nu, \beta, \mu, k_{1}, \rho, a, \epsilon\right)} \\
& \quad \leq \frac{C_{1} C_{2}}{(2 \pi)^{1 / 2} C_{P}\left(r_{Q, R_{D}}\right)^{\frac{1}{k_{1} \delta_{D}}}|\epsilon|^{\Delta_{l}} a^{\delta_{l}}\left\|A_{l}(m, \epsilon)\right\|_{(\beta, \mu)}|| \omega\left(\tau_{1}, \tau_{2}, m\right) \|_{\left(\nu, \beta, \mu, k_{1}, \rho, a, \epsilon\right)}} \leq C_{1} C_{2} \\
& (2 \pi)^{1 / 2} C_{P}\left(r_{Q, R_{D}}\right)^{\frac{1}{k_{1} \delta_{D}}} \epsilon_{0}^{\Delta_{l}} a^{\delta_{l}} \mathbf{A}_{l, \epsilon_{0}} \varpi .
\end{aligned}
$$


Moreover, assumed from the second and third inequalities of (13) together with (14), (16) and (17), we reach

$$
\begin{aligned}
& \| \epsilon^{\Delta_{l}-d_{l}} \frac{\tau_{1}^{k_{1}}}{P_{m}\left(\tau_{1}\right)} \int_{0}^{\tau_{1}^{k_{1}}}\left(\tau_{1}^{k_{1}}-s_{1}\right)^{\frac{d_{l, q}}{k_{1}}-1}(-1)^{p_{2}} \tau_{2}^{p_{2}} s_{1}^{q-1} \\
& \quad \times\left(\frac{1}{(2 \pi)^{1 / 2}} \int_{-\infty}^{+\infty} A_{l}\left(m-m_{1}, \epsilon\right) R_{l}\left(i m_{1}\right) \omega\left(s_{1}^{1 / k_{1}}, \tau_{2}, m_{1}\right) d m_{1}\right) d s_{1} \|_{\left(\nu, \beta, \mu, k_{1}, \rho, a, \epsilon\right)} \\
& \quad=\| \epsilon^{\Delta_{l}-d_{l}} \frac{R_{D}(i m)}{P_{m}\left(\tau_{1}\right)} \tau_{1}^{k_{1}} \int_{0}^{\tau_{1}^{k_{1}}}\left(\tau_{1}^{k_{1}}-s_{1}\right)^{\frac{d_{l, q}}{k_{1}}-1}(-1)^{p_{2}} \tau_{2}^{p_{2}} s_{1}^{q-1} \\
& \times\left(\frac{1}{(2 \pi)^{1 / 2} R_{D}(i m)} \int_{-\infty}^{+\infty} A_{l}\left(m-m_{1}, \epsilon\right)^{R_{l}}\left(i m_{1}\right) \omega\left(s_{1}^{1 / k_{1}}, \tau_{2}, m_{1}\right) d m_{1}\right) d s_{1} \|_{\left(\nu, \beta, \mu, k_{1}, \rho, a, \epsilon\right)} \\
& \quad \times\left\|\frac{C_{1}\left(r_{Q, R_{D}}\right)^{\frac{1}{k_{1} \delta_{D}}}|\epsilon|^{\Delta_{l}-d_{l}}|\epsilon|^{d_{l, q}} a^{p_{2}}}{(2 \pi)^{1 / 2} R_{D}(i m)} \int_{-\infty}^{+\infty} A_{l}\left(m-m_{1}, \epsilon\right) R_{l}\left(i m_{1}\right) \omega\left(\tau_{1}, \tau_{2}, m_{1}\right) d m_{1}\right\|_{\left(\nu, \beta, \mu, k_{1}, \rho, a, \epsilon\right)} \\
& \leq \frac{C_{1} C_{2}}{(2 \pi)^{1 / 2} C_{P}\left(r_{Q, R_{D}}\right)^{\frac{1}{k_{1} \delta_{D}}}|\epsilon|^{\Delta_{l}-q k_{1}} a^{p_{2}}\left\|A_{l}(m, \epsilon)\right\|}\left\|_{(\beta, \mu)}\right\| \omega\left(\tau_{1}, \tau_{2}, m\right) \|_{\left(\nu, \beta, \mu, k_{1}, \rho, a, \epsilon\right)} \\
& \leq \frac{C_{1} C_{2}}{(2 \pi)^{1 / 2} C_{P}\left(r_{Q, R_{D}}\right)^{\frac{1}{k_{1} \delta_{D}}}} \epsilon_{0}^{\Delta_{l}-q k_{1}} a^{p_{2}} \mathbf{A}_{l, \epsilon_{0}} \varpi
\end{aligned}
$$

provided that $1 \leq q \leq \delta_{l}$ along with

$$
\begin{aligned}
& \| \epsilon^{\Delta_{l}-d_{l}} \frac{\tau_{1}^{k_{1}}}{P_{m}\left(\tau_{1}\right)} \int_{0}^{\tau_{1}^{k_{1}}}\left(\tau_{1}^{k_{1}}-s_{1}\right)^{\frac{d_{l, q}+k_{1}(q-p)}{k_{1}}-1}(-1)^{p_{2}} \tau_{2}^{p_{2}} s_{1}^{p-1} \\
& \quad \times\left(\frac{1}{(2 \pi)^{1 / 2}} \int_{-\infty}^{+\infty} A_{l}\left(m-m_{1}, \epsilon\right) R_{l}\left(i m_{1}\right) \omega\left(s_{1}^{1 / k_{1}}, \tau_{2}, m_{1}\right) d m_{1}\right) d s_{1} \|_{\left(\nu, \beta, \mu, k_{1}, \rho, a, \epsilon\right)} \\
& \quad=\| \epsilon^{\Delta_{l}-d_{l}} \frac{R_{D}(i m)}{P_{m}\left(\tau_{1}\right)} \tau_{1}^{k_{1}} \int_{0}^{\tau_{1}^{k_{1}}}\left(\tau_{1}^{k_{1}}-s_{1}\right)^{\frac{d_{l, q}+k_{1}(q-p)}{k_{1}}-1}(-1)^{p_{2}} \tau_{2}^{p_{2}} s_{1}^{p-1} \\
& \times\left(\frac{1}{(2 \pi)^{1 / 2} R_{D}(i m)} \int_{-\infty}^{+\infty} A_{l}\left(m-m_{1}, \epsilon\right) R_{l}\left(i m_{1}\right) \omega\left(s_{1}^{1 / k_{1}}, \tau_{2}, m_{1}\right) d m_{1}\right) d s_{1} \|_{\left(\nu, \beta, \mu, k_{1}, \rho, a, \epsilon\right)} \\
& \quad \times\left\|\frac{C_{P}\left(r_{Q, R_{D}}\right)^{\frac{1}{k_{1} \delta_{D}}}|\epsilon|^{\Delta_{l}-d_{l}}|\epsilon|^{d_{l, q}+k_{1}(q-p)} a^{p_{2}}}{(2 \pi)^{1 / 2} R_{D}(i m)} \int_{-\infty}^{+\infty} A_{l}\left(m-m_{1}, \epsilon\right) R_{l}\left(i m_{1}\right) \omega\left(\tau_{1}, \tau_{2}, m_{1}\right) d m_{1}\right\|_{\left(\nu, \beta, \mu, k_{1}, \rho, a, \epsilon\right)} \\
& \leq \frac{C_{1} C_{2}}{(2 \pi)^{1 / 2} C_{P}\left(r_{Q}, R_{D}\right)^{\frac{1}{k_{1} \delta_{D}}}}|\epsilon|^{\Delta_{l}-p k_{1}} a^{p_{2}}\left\|A_{l}(m, \epsilon)\right\|(\beta, \mu)\left\|\omega\left(\tau_{1}, \tau_{2}, m\right)\right\|_{\left(\nu, \beta, \mu, k_{1}, \rho, a, \epsilon\right)} \\
& \leq \frac{C_{1} C_{2}}{(2 \pi)^{1 / 2} C_{P}\left(r_{Q, R_{D}}\right)^{\frac{1}{k_{1} \delta_{D}}}} \epsilon_{0}^{\Delta_{l}-p k_{1} a^{p_{2}} \mathbf{A}_{l, \epsilon_{0}} \varpi}
\end{aligned}
$$

whenever $1 \leq p \leq q-1$ and $1 \leq q \leq \delta_{l}$.

At last, we need to control the norm of the last term of the map $\mathcal{H}_{\epsilon}$. Indeed, according to 
the upper and lower bounds (18), (61), we achieve

$$
\begin{aligned}
& \left\|\frac{\mathcal{F}\left(\tau_{1}, \tau_{2}, m, \epsilon\right)}{P_{m}\left(\tau_{1}\right)}\right\|_{\left(\nu, \beta, \mu, k_{1}, \rho, a, \epsilon\right)}=\sup _{\substack{\tau_{1} \in S_{d_{1}} \cup D_{\rho}, \tau_{2} \in D_{a} \\
m \in \mathbb{R}}}\left|\frac{1}{P_{m}\left(\tau_{1}\right)} \sum_{j_{1} \in J_{1}, j_{2} \in J_{2}} \mathcal{F}_{j_{1}, j_{2}}(m, \epsilon) \tau_{1}^{j_{1}} \tau_{2}^{j_{2}}\right| \\
& \quad \times(1+|m|)^{\mu} e^{\beta|m|}\left|\frac{\epsilon}{\tau_{1}}\right| \exp \left(-\nu\left|\frac{\tau_{1}}{\epsilon}\right|^{k_{1}}\right) \frac{1}{\left|\tau_{2}\right|} \leq \frac{1}{C_{P}\left(r_{Q, R_{D}}\right)^{\frac{1}{k_{1} \delta_{D}}}} \frac{1}{\min _{m \in \mathbb{R}}\left|R_{D}(i m)\right|} \\
& \quad \times \sum_{j_{1} \in J_{1}, j_{2} \in J_{2}}|| \mathcal{F}_{j_{1}, j_{2}}(m, \epsilon) \|_{(\beta, \mu)} \sup _{\tau_{1} \in S_{d_{1}} \cup D_{\rho}}|\epsilon|^{j_{1}}\left|\frac{\tau_{1}}{\epsilon}\right|^{j_{1}-1} \exp \left(-\nu\left|\frac{\tau_{1}}{\epsilon}\right|^{k_{1}}\right) a^{j_{2}-1} \\
& \leq \frac{1}{C_{P}\left(r_{Q, R_{D}}\right)^{\frac{1}{k_{1} \delta_{D}}}} \frac{1}{\min _{m \in \mathbb{R}}\left|R_{D}(i m)\right|} \sum_{j_{1} \in J_{1}, j_{2} \in J_{2}} \mathbf{F}_{j_{1}, j_{2}, \epsilon_{0}} \epsilon_{0}^{j_{1}} \sup _{x \geq 0} x^{j_{1}-1} \exp \left(-\nu x^{k_{1}}\right) a^{j_{2}-1} .
\end{aligned}
$$

Now, we select $\epsilon_{0}>0$ close enough to 0 and take suitably $\varpi>0$ in a way that the next inequality

$$
\begin{aligned}
& \frac{C_{1}}{C_{P}\left(r_{Q, R_{D}}\right)^{\frac{1}{k_{1} \delta_{D}}}} \frac{1}{\Gamma\left(\frac{d_{D}}{k_{1}}\right)} a^{\delta_{D}} \epsilon_{0}^{d_{D}} \varpi+\sum_{\substack{p_{1}+p_{2}=\delta_{D} \\
1 \leq p_{1}<\delta_{D}}} \frac{\delta_{D} !}{p_{1} ! p_{2} !}\left(\sum_{q=1}^{p_{1}}\left|a_{q, p_{1}}\right|\right. \\
& \times\left[\frac{1}{\Gamma\left(\frac{d_{D, q}}{k_{1}}\right)} \frac{C_{1}}{C_{P}\left(r_{Q, R_{D}}\right)^{\frac{1}{k_{1} \delta_{D}}}} a^{p_{2}} \epsilon_{0}^{d_{D, q}} k_{1}^{q} \varpi\right. \\
& \left.\left.+\sum_{1 \leq p \leq q-1}\left|A_{q, p}\right| \frac{1}{\Gamma\left(\frac{d_{D, q}+k_{1}(q-p)}{k_{1}}\right)} k_{1}^{p} \frac{C_{1}}{C_{P}\left(r_{Q, R_{D}}\right)^{\frac{1}{k_{1} \delta_{D}}}} a^{p_{2}} \epsilon_{0}^{d_{D, q}+k_{1}(q-p)} \varpi\right]\right) \\
& +\sum_{q=1}^{\delta_{D}-1}\left|a_{q, \delta_{D}}\right|\left[\frac{1}{\Gamma\left(\frac{d_{D, q}}{k_{1}}\right)} k_{1}^{q} \frac{C_{1}}{C_{P}\left(r_{Q, R_{D}}\right)^{\frac{1}{k_{1} \delta_{D}}}} \epsilon_{0}^{d_{D, q}} \varpi\right. \\
& \left.+\sum_{1 \leq p \leq q-1}\left|A_{q, p}\right| \frac{1}{\Gamma\left(\frac{d_{D, q}+k_{1}(q-p)}{k_{1}}\right)} k_{1}^{p} \frac{C_{1}}{C_{P}\left(r_{Q, R_{D}}\right)^{\frac{1}{k_{1} \delta_{D}}}} \epsilon_{0}^{d_{D, q}+k_{1}(q-p)} \varpi\right] \\
& +\sum_{1 \leq p \leq \delta_{D}-1}\left|A_{\delta_{D}, p}\right| \frac{1}{\Gamma\left(\delta_{D}-p\right)} k_{1}^{p} \frac{C_{1}}{C_{P}\left(r_{Q, R_{D}}\right)^{\frac{1}{k_{1} \delta_{D}}}} \epsilon_{0}^{k_{1}\left(\delta_{D}-p\right)} \varpi \\
& +\sum_{l=1}^{D-1}\left[\frac{1}{\Gamma\left(\frac{d_{l}}{k_{1}}\right)} \frac{C_{1} C_{2}}{(2 \pi)^{1 / 2} C_{P}\left(r_{Q, R_{D}}\right)^{\frac{1}{k_{1} \delta_{D}}}} \epsilon_{0}^{\Delta_{l}} a^{\delta_{l}} \mathbf{A}_{l, \epsilon_{0}} \varpi\right. \\
& +\sum_{\substack{p_{1}+p_{2}=\delta_{l} \\
1 \leq p_{1} \leq \delta_{l}}} \frac{\delta_{l} !}{p_{1} ! p_{2} !}\left(\sum _ { q = 1 } ^ { p _ { 1 } } | a _ { q , p _ { 1 } } | \left[\frac{k_{1}^{q}}{\Gamma\left(\frac{d_{l, q}}{k_{1}}\right)} \frac{C_{1} C_{2}}{(2 \pi)^{1 / 2} C_{P}\left(r_{Q, R_{D}}\right)^{\frac{1}{k_{1} \delta_{D}}}} \epsilon_{0}^{\Delta_{l}-q k_{1}} a^{p_{2}} \mathbf{A}_{l, \epsilon_{0}} \varpi\right.\right. \\
& \left.\left.\left.+\sum_{1 \leq p \leq q-1}\left|A_{q, p}\right| \frac{k_{1}^{p}}{\Gamma\left(\frac{d_{l, q}+k_{1}(q-p)}{k_{1}}\right)} \frac{C_{1} C_{2}}{(2 \pi)^{1 / 2} C_{P}\left(r_{Q, R_{D}}\right)^{\frac{1}{k_{1} \delta_{D}}}} \epsilon_{0}^{\Delta_{l}-p k_{1}} a^{p_{2}} \mathbf{A}_{l, \epsilon_{0}} \varpi\right]\right)\right] \\
& +\frac{1}{C_{P}\left(r_{Q, R_{D}}\right)^{\frac{1}{k_{1} \delta_{D}}}} \frac{1}{\min _{m \in \mathbb{R}}\left|R_{D}(i m)\right|} \sum_{j_{1} \in J_{1}, j_{2} \in J_{2}} \mathbf{F}_{j_{1}, j_{2}, \epsilon_{0}} \epsilon_{0}^{j_{1}} \sup _{x \geq 0} x^{j_{1}-1} \exp \left(-\nu x^{k_{1}}\right) a^{j_{2}-1} \leq \varpi
\end{aligned}
$$

holds.

Eventually, the collection of the ten above bounds (69), (70), (71), (72), (73), (74), (75), (76), (77), (78) under the restriction (79) prompt the due inclusion (67).

We turn to the second item addressing the $1 / 2-$ Lipschitz feature (68). Let $\omega_{1}, \omega_{2}$ be elements of the closed ball $\bar{B}_{\varpi}$ in $F_{\left(\nu, \beta, \mu, k_{1}, \rho, a, \epsilon\right)}^{d_{1}}$ where the radius $\varpi$ has been fixed in the first item. 
We display norm estimates for each block of the difference $\mathcal{H}_{\epsilon}\left(\omega_{1}\right)-\mathcal{H}_{\epsilon}\left(\omega_{2}\right)$. Drew on the above nine upper bounds (69), (70), (71), (72), (73), (74), (75), (76), (77), we deduce the next list of inequalities

$$
\begin{aligned}
& \| \frac{\tau_{1}^{k_{1}}}{P_{m}\left(\tau_{1}\right)} \int_{0}^{\tau_{1}^{k_{1}}}\left(\tau_{1}^{k_{1}}-s_{1}\right)^{\frac{d_{D}}{k_{1}}-1} \tau_{2}^{\delta_{D}}(-1)^{\delta_{D}} R_{D}(i m) \\
& \quad \times\left(\omega_{1}\left(s_{1}^{1 / k_{1}}, \tau_{2}, m\right)-\omega_{2}\left(s_{1}^{1 / k_{1}}, \tau_{2}, m\right)\right) \frac{d s_{1}}{s_{1}} \|_{\left(\nu, \beta, \mu, k_{1}, \rho, a, \epsilon\right)} \\
& \quad \leq \frac{C_{1}}{C_{P}\left(r_{Q, R_{D}}\right)^{\frac{1}{k_{1} \delta_{D}}}} a^{\delta_{D}} \epsilon_{0}^{d_{D}}\left\|\omega_{1}\left(\tau_{1}, \tau_{2}, m\right)-\omega_{2}\left(\tau_{1}, \tau_{2}, m\right)\right\|_{\left(\nu, \beta, \mu, k_{1}, \rho, a, \epsilon\right)}
\end{aligned}
$$

and

$$
\begin{aligned}
& \| \frac{\tau_{1}^{k_{1}}}{P_{m}\left(\tau_{1}\right)} \int_{0}^{\tau_{1}^{k_{1}}}\left(\tau_{1}^{k_{1}}-s_{1}\right)^{\frac{d_{D, q}}{k_{1}}-1}(-1)^{p_{2}} \tau_{2}^{p_{2}} R_{D}(i m) s_{1}^{q-1} \\
& \quad \times\left(\omega_{1}\left(s_{1}^{1 / k_{1}}, \tau_{2}, m\right)-\omega_{2}\left(s_{1}^{1 / k_{1}}, \tau_{2}, m\right)\right) d s_{1} \|_{\left(\nu, \beta, \mu, k_{1}, \rho, a, \epsilon\right)} \\
& \quad \leq \frac{C_{1}}{C_{P}\left(r_{Q, R_{D}}\right)^{\frac{1}{k_{1} \delta_{D}}}} a^{p_{2}} \epsilon_{0}^{d_{D, q}}\left\|\omega_{1}\left(\tau_{1}, \tau_{2}, m\right)-\omega_{2}\left(\tau_{1}, \tau_{2}, m\right)\right\|_{\left(\nu, \beta, \mu, k_{1}, \rho, a, \epsilon\right)}
\end{aligned}
$$

for $1 \leq q \leq \delta_{D}-1$ along with

$$
\begin{aligned}
\| \frac{\tau_{1}^{k_{1}}}{P_{m}\left(\tau_{1}\right)} & \int_{0}^{\tau_{1}^{k_{1}}}\left(\tau_{1}^{k_{1}}-s_{1}\right)^{\frac{d_{D, q}+k_{1}(q-p)}{k_{1}}}-1(-1)^{p_{2}} \tau_{2}^{p_{2}} \\
& \times R_{D}(i m) s_{1}^{p-1}\left(\omega_{1}\left(s_{1}^{1 / k_{1}}, \tau_{2}, m\right)-\omega_{2}\left(s_{1}^{1 / k_{1}}, \tau_{2}, m\right)\right) d s_{1} \|_{\left(\nu, \beta, \mu, k_{1}, \rho, a, \epsilon\right)} \\
& \leq \frac{C_{1}}{C_{P}\left(r_{Q, R_{D}}\right)^{\frac{1}{k_{1} \delta_{D}}}} a^{p_{2}} \epsilon_{0}^{d_{D, q}+k_{1}(q-p)}\left\|\omega_{1}\left(\tau_{1}, \tau_{2}, m\right)-\omega_{2}\left(\tau_{1}, \tau_{2}, m\right)\right\|_{\left(\nu, \beta, \mu, k_{1}, \rho, a, \epsilon\right)}
\end{aligned}
$$

provided that $1 \leq p \leq q-1$ and $1 \leq q \leq \delta_{D}-1$ and

$$
\begin{aligned}
& \| \frac{\tau_{1}^{k_{1}}}{P_{m}\left(\tau_{1}\right)} \int_{0}^{\tau_{1}^{k_{1}}}\left(\tau_{1}^{k_{1}}-s_{1}\right)^{\frac{d_{D, q}}{k_{1}}-1} s_{1}^{q-1} R_{D}(i m) \\
& \quad \times\left(\omega_{1}\left(s_{1}^{1 / k_{1}}, \tau_{2}, m\right)-\omega_{2}\left(s_{1}^{1 / k_{1}}, \tau_{2}, m\right)\right) d s_{1} \|_{\left(\nu, \beta, \mu, k_{1}, \rho, a, \epsilon\right)} \\
& \quad \leq \frac{C_{1}}{C_{P}\left(r_{Q, R_{D}}\right)^{\frac{1}{k_{1} \delta_{D}}}} \epsilon_{0}^{d_{D, q}}\left\|\omega_{1}\left(\tau_{1}, \tau_{2}, m\right)-\omega_{2}\left(\tau_{1}, \tau_{2}, m\right)\right\|_{\left(\nu, \beta, \mu, k_{1}, \rho, a, \epsilon\right)}
\end{aligned}
$$

for $1 \leq q \leq \delta_{D}-1$ together with

$$
\begin{aligned}
\| \frac{\tau_{1}^{k_{1}}}{P_{m}\left(\tau_{1}\right)} \int_{0}^{\tau_{1}^{k_{1}}} & \left(\tau_{1}^{k_{1}}-s_{1}\right)^{\frac{d_{D, q}+k_{1}(q-p)}{k_{1}}}-1 s_{1}^{p-1} R_{D}(i m) \\
& \times\left(\omega_{1}\left(s_{1}^{1 / k_{1}}, \tau_{2}, m\right)-\omega_{2}\left(s_{1}^{1 / k_{1}}, \tau_{2}, m\right)\right) d s_{1} \|_{\left(\nu, \beta, \mu, k_{1}, \rho, a, \epsilon\right)} \\
& \leq \frac{C_{1}}{C_{P}\left(r_{Q, R_{D}}\right)^{\frac{1}{k_{1} \delta_{D}}}} \epsilon_{0}^{d_{D, q}+k_{1}(q-p)}\left\|\omega_{1}\left(\tau_{1}, \tau_{2}, m\right)-\omega_{2}\left(\tau_{1}, \tau_{2}, m\right)\right\|_{\left(\nu, \beta, \mu, k_{1}, \rho, a, \epsilon\right)}
\end{aligned}
$$


whenever $1 \leq p \leq q-1,1 \leq q \leq \delta_{D}-1$ and

$$
\begin{aligned}
\| \frac{\tau_{1}^{k_{1}}}{P_{m}\left(\tau_{1}\right)} \int_{0}^{\tau_{1}^{k_{1}}}\left(\tau_{1}^{k_{1}}-s_{1}\right)^{\delta_{D}-p-1} R_{D}(i m) s_{1}^{p-1} \\
\quad\left(\omega_{1}\left(s_{1}^{1 / k_{1}}, \tau_{2}, m\right)-\omega_{2}\left(s_{1}^{1 / k_{1}}, \tau_{2}, m\right)\right) d s_{1} \|_{\left(\nu, \beta, \mu, k_{1}, \rho, a, \epsilon\right)} \\
\quad \leq \frac{C_{1}}{C_{P}\left(r_{Q, R_{D}}\right)^{\frac{1}{k_{1} \delta_{D}}}} \epsilon_{0}^{k_{1}\left(\delta_{D}-p\right)}\left\|\omega_{1}\left(\tau_{1}, \tau_{2}, m\right)-\omega_{2}\left(\tau_{1}, \tau_{2}, m\right)\right\|_{\left(\nu, \beta, \mu, k_{1}, \rho, a, \epsilon\right)}
\end{aligned}
$$

for all $1 \leq p \leq \delta_{D}-1$. Furthermore,

$$
\begin{aligned}
& \| \epsilon^{\Delta_{l}-d_{l}} \frac{\tau_{1}^{k_{1}}}{P_{m}\left(\tau_{1}\right)} \int_{0}^{\tau_{1}^{k_{1}}}\left(\tau_{1}^{k_{1}}-s_{1}\right)^{\frac{d_{l}}{k_{1}}-1} \tau_{2}^{\delta_{l}}(-1)^{\delta_{l}} s_{1}^{-1} \\
& \quad \times\left(\frac{1}{(2 \pi)^{1 / 2}} \int_{-\infty}^{+\infty} A_{l}\left(m-m_{1}, \epsilon\right) R_{l}\left(i m_{1}\right)\right. \\
& \left.\quad \times\left(\omega_{1}\left(s_{1}^{1 / k_{1}}, \tau_{2}, m_{1}\right)-\omega_{2}\left(s_{1}^{1 / k_{1}}, \tau_{2}, m_{1}\right)\right) d m_{1}\right) d s_{1} \|_{\left(\nu, \beta, \mu, k_{1}, \rho, a, \epsilon\right)} \\
& \leq \frac{C_{1} C_{2}}{(2 \pi)^{1 / 2} C_{P}\left(r_{Q, R_{D}}\right)^{\frac{1}{k_{1} \delta_{D}}}} \epsilon_{0}^{\Delta_{l}} a^{\delta_{l}} \mathbf{A}_{l, \epsilon_{0}}\left\|\omega_{1}\left(\tau_{1}, \tau_{2}, m\right)-\omega_{2}\left(\tau_{1}, \tau_{2}, m\right)\right\|_{\left(\nu, \beta, \mu, k_{1}, \rho, a, \epsilon\right)}
\end{aligned}
$$

holds as well as

$$
\begin{aligned}
& \| \epsilon^{\Delta_{l}-d_{l}} \frac{\tau_{1}^{k_{1}}}{P_{m}\left(\tau_{1}\right)} \int_{0}^{\tau_{1}^{k_{1}}}\left(\tau_{1}^{k_{1}}-s_{1}\right)^{\frac{d_{l, q}}{k_{1}}-1}(-1)^{p_{2}} \tau_{2}^{p_{2}} s_{1}^{q-1} \\
& \quad \times\left(\frac{1}{(2 \pi)^{1 / 2}} \int_{-\infty}^{+\infty} A_{l}\left(m-m_{1}, \epsilon\right) R_{l}\left(i m_{1}\right)\right. \\
& \leq \frac{\left.\left(\omega_{1}\left(s_{1}^{1 / k_{1}}, \tau_{2}, m_{1}\right)-\omega_{2}\left(s_{1}^{1 / k_{1}}, \tau_{2}, m_{1}\right)\right) d m_{1}\right) d s_{1} \|_{\left(\nu, \beta, \mu, k_{1}, \rho, a, \epsilon\right)}}{(2 \pi)^{1 / 2} C_{P}\left(r_{Q, R_{D}}\right)^{\frac{1}{k_{1} \delta_{D}}}} \epsilon_{0}^{\Delta_{l}-q k_{1}} a^{p_{2}} \mathbf{A}_{l, \epsilon_{0}}\left\|\omega_{1}\left(\tau_{1}, \tau_{2}, m\right)-\omega_{2}\left(\tau_{1}, \tau_{2}, m\right)\right\|_{\left(\nu, \beta, \mu, k_{1}, \rho, a, \epsilon\right)}
\end{aligned}
$$

provided that $1 \leq q \leq \delta_{l}$ and

$$
\begin{aligned}
& \| \epsilon^{\Delta_{l}-d_{l}} \frac{\tau_{1}^{k_{1}}}{P_{m}\left(\tau_{1}\right)} \int_{0}^{\tau_{1}^{k_{1}}}\left(\tau_{1}^{k_{1}}-s_{1}\right)^{\frac{d_{l, q}+k_{1}(q-p)}{k_{1}}-1}(-1)^{p_{2}} \tau_{2}^{p_{2}} s_{1}^{p-1} \\
& \quad \times\left(\frac{1}{(2 \pi)^{1 / 2}} \int_{-\infty}^{+\infty} A_{l}\left(m-m_{1}, \epsilon\right) R_{l}\left(i m_{1}\right)\right. \\
& \left.\quad \times\left(\omega_{1}\left(s_{1}^{1 / k_{1}}, \tau_{2}, m_{1}\right)-\omega_{2}\left(s_{1}^{1 / k_{1}}, \tau_{2}, m_{1}\right)\right) d m_{1}\right) d s_{1} \|_{\left(\nu, \beta, \mu, k_{1}, \rho, a, \epsilon\right)} \\
& \leq \frac{C_{1} C_{2}}{(2 \pi)^{1 / 2} C_{P}\left(r_{Q, R_{D}}\right)^{\frac{1}{k_{1} \delta_{D}}}} \epsilon_{0}^{\Delta_{l}-p k_{1}} a^{p_{2}} \mathbf{A}_{l, \epsilon_{0}}\left\|\omega_{1}\left(\tau_{1}, \tau_{2}, m\right)-\omega_{2}\left(\tau_{1}, \tau_{2}, m\right)\right\|_{\left(\nu, \beta, \mu, k_{1}, \rho, a, \epsilon\right)}
\end{aligned}
$$

whenever $1 \leq p \leq q-1$ and $1 \leq q \leq \delta_{l}$. 
We prescribe $\epsilon_{0}>0$ small enough allowing the next inequality

$$
\begin{aligned}
& \frac{C_{1}}{C_{P}\left(r_{Q, R_{D}}\right)^{\frac{1}{k_{1} \delta_{D}}}} \frac{1}{\Gamma\left(\frac{d_{D}}{k_{1}}\right)} a^{\delta_{D}} \epsilon_{0}^{d_{D}}+\sum_{\substack{p_{1}+p_{2}=\delta_{D} \\
1 \leq p_{1}<\delta_{D}}} \frac{\delta_{D} !}{p_{1} ! p_{2} !}\left(\sum_{q=1}^{p_{1}}\left|a_{q, p_{1}}\right|\right. \\
& \times\left[\frac{1}{\Gamma\left(\frac{d_{D, q}}{k_{1}}\right)} \frac{C_{1}}{C_{P}\left(r_{Q, R_{D}}\right)^{\frac{1}{k_{1} \delta_{D}}}} a^{p_{2}} \epsilon_{0}^{d_{D, q}} k_{1}^{q}\right. \\
& \left.\left.+\sum_{1 \leq p \leq q-1}\left|A_{q, p}\right| \frac{1}{\Gamma\left(\frac{d_{D, q}+k_{1}(q-p)}{k_{1}}\right)} k_{1}^{p} \frac{C_{1}}{C_{P}\left(r_{Q, R_{D}}\right)^{\frac{1}{k_{1} \delta_{D}}}} a^{p_{2}} \epsilon_{0}^{d_{D, q}+k_{1}(q-p)}\right]\right) \\
& +\sum_{q=1}^{\delta_{D}-1}\left|a_{q, \delta_{D}}\right|\left[\frac{1}{\Gamma\left(\frac{d_{D, q}}{k_{1}}\right)} k_{1}^{q} \frac{C_{1}}{C_{P}\left(r_{Q, R_{D}}\right)^{\frac{1}{k_{1} \delta_{D}}}} \epsilon_{0}^{d_{D, q}}\right. \\
& \left.+\sum_{1 \leq p \leq q-1}\left|A_{q, p}\right| \frac{1}{\Gamma\left(\frac{d_{D, q}+k_{1}(q-p)}{k_{1}}\right)} k_{1}^{p} \frac{C_{1}}{C_{P}\left(r_{Q, R_{D}}\right)^{\frac{1}{k_{1} \delta_{D}}}} \epsilon_{0}^{d_{D, q}+k_{1}(q-p)}\right] \\
& +\sum_{1 \leq p \leq \delta_{D}-1}\left|A_{\delta_{D}, p}\right| \frac{1}{\Gamma\left(\delta_{D}-p\right)} k_{1}^{p} \frac{C_{1}}{C_{P}\left(r_{Q, R_{D}}\right)^{\frac{1}{k_{1} \delta_{D}}}} \epsilon_{0}^{k_{1}\left(\delta_{D}-p\right)} \\
& +\sum_{l=1}^{D-1}\left[\frac{1}{\Gamma\left(\frac{d_{l}}{k_{1}}\right)} \frac{C_{1} C_{2}}{(2 \pi)^{1 / 2} C_{P}\left(r_{Q, R_{D}}\right)^{\frac{1}{k_{1} \delta_{D}}}} \epsilon_{0}^{\Delta_{l}} a^{\delta_{l}} \mathbf{A}_{l, \epsilon_{0}}\right. \\
& +\sum_{\substack{p_{1}+p_{2}=\delta_{l} \\
1 \leq p_{1} \leq \delta_{l}}} \frac{\delta_{l} !}{p_{1} ! p_{2} !}\left(\sum _ { q = 1 } ^ { p _ { 1 } } | a _ { q , p _ { 1 } } | \left[\frac{k_{1}^{q}}{\Gamma\left(\frac{d_{l, q}}{k_{1}}\right)} \frac{C_{1} C_{2}}{(2 \pi)^{1 / 2} C_{P}\left(r_{Q, R_{D}}\right)^{\frac{1}{k_{1} \delta_{D}}}} \epsilon_{0}^{\Delta_{l}-q k_{1}} a^{p_{2}} \mathbf{A}_{l, \epsilon_{0}}\right.\right. \\
& \left.\left.\left.+\sum_{1 \leq p \leq q-1}\left|A_{q, p}\right| \frac{k_{1}^{p}}{\Gamma\left(\frac{d_{l, q}+k_{1}(q-p)}{k_{1}}\right)} \frac{C_{1} C_{2}}{(2 \pi)^{1 / 2} C_{P}\left(r_{Q, R_{D}}\right)^{\frac{1}{k_{1} \delta_{D}}}} \epsilon_{0}^{\Delta_{l}-p k_{1}} a^{p_{2}} \mathbf{A}_{l, \epsilon_{0}}\right]\right)\right] \leq 1 / 2
\end{aligned}
$$

to hold.

The combination of the nine above bounds (80), (81), (82), (83), (84), (85), (86), (87), (88) subjected to (89) triggers the shrinking property (68) of the map $\mathcal{H}_{\epsilon}$.

At last, we choose $\varpi>0$ as in the first item and $\epsilon_{0}>0$ conforming to both (79) and (89). For these given values, the map $\mathcal{H}_{\epsilon}$ enjoys conjointly both properties (67) and (68) for all $\epsilon \in D_{\epsilon_{0}} \backslash\{0\}$. The proposition follows.

The next proposition provides a solution to the associated convolution equation displayed in (42) within the Banach space given in Definition 4.

Proposition 4 Let us choose an appropriate inner radius $r_{Q, R_{D}}>0$ and aperture $\eta_{Q, R_{D}}>0$ of the sector $S_{Q, R_{D}}$ defined in (15), together with an unbounded sector $S_{d_{1}}$ and a radius $\rho$ that conform the requirements of Lemma 4.

Then, a small radius $\epsilon_{0}>0$ and a constant $\varpi>0$ can be singled out in a manner that for all $\epsilon \in D_{\epsilon_{0}} \backslash\{0\}$, a unique solution $\omega_{d_{1}}\left(\tau_{1}, \tau_{2}, m, \epsilon\right)$ of (42) exists such that

- the map $\left(\tau_{1}, \tau_{2}, m\right) \mapsto \omega_{d_{1}}\left(\tau_{1}, \tau_{2}, m, \epsilon\right)$ belongs to $F_{\left(\nu, \beta, \mu, k_{1}, \rho, a, \epsilon\right)}^{d_{1}}$ under the constraint

$$
\sup _{\epsilon \in D_{\epsilon_{0}} \backslash\{0\}}\left\|\omega_{d_{1}}\left(\tau_{1}, \tau_{2}, m, \epsilon\right)\right\|_{\left(\nu, \beta, \mu, k_{1}, \rho, a, \epsilon\right)} \leq \varpi
$$


- the partial map $\epsilon \mapsto \omega_{d_{1}}\left(\tau_{1}, \tau_{2}, m, \epsilon\right)$ represents an analytic map from $D_{\epsilon_{0}} \backslash\{0\}$ into $\mathbb{C}$, for any given $\tau_{1} \in S_{d_{1}} \cup D_{\rho}, \tau_{2} \in D_{a}$ and $m \in \mathbb{R}$.

Proof We select $\epsilon_{0}>0$ and $\varpi>0$ as in Proposition 3. We set the closed ball $\bar{B}_{\varpi} \subset F_{\left(\nu, \beta, \mu, k_{1}, \rho, a, \epsilon\right)}^{d_{1}}$ which represents a complete metric space for the distance $d(x, y)=\|x-y\|_{\left(\nu, \beta, \mu, k_{1}, \rho, a, \epsilon\right)}$. The proposition 3 claims that $\mathcal{H}_{\epsilon}$ induces a contractive map from $\left(\bar{B}_{\varpi}, d\right)$ into itself. Then, according to the classical Banach fixed point theorem, $\mathcal{H}_{\epsilon}$ possesses a unique fixed point $\omega_{d_{1}}\left(\tau_{1}, \tau_{2}, m, \epsilon\right)$, meaning that

$$
\mathcal{H}_{\epsilon}\left(\omega_{d_{1}}\left(\tau_{1}, \tau_{2}, m, \epsilon\right)\right)=\omega_{d_{1}}\left(\tau_{1}, \tau_{2}, m, \epsilon\right)
$$

that belongs to the ball $\bar{B}_{\varpi}$ for all $\epsilon \in D_{\epsilon_{0}} \backslash\{0\}$. Furthermore, the map $\omega_{d_{1}}\left(\tau_{1}, \tau_{2}, m, \epsilon\right)$ relies holomorphically on $\epsilon$ since $\mathcal{H}_{\epsilon}$ does on the domain $D_{\epsilon_{0}} \backslash\{0\}$.

Moving the term $\left(k_{1} \tau_{1}^{k_{1}}\right)^{\delta_{D}} R_{D}(i m) \omega_{d_{1}}\left(\tau_{1}, \tau_{2}, m, \epsilon\right)$ from the right to the left handside of (42), we observe by dividing by the function $P_{m}\left(\tau_{1}\right)$ defined in $(60)$ that the convolution equation (42) can be exactly rearranged as the equation (91) above. As a result, the unique fixed point $\omega_{d_{1}}\left(\tau_{1}, \tau_{2}, m, \epsilon\right)$ of $\mathcal{H}_{\epsilon}$ obtained in $\bar{B}_{\varpi}$ precisely solves the equation (42). This yields the statement of Proposition 4.

In the ensuing proposition, we come up with analytic solutions to the auxiliary problem (30).

Proposition 5 For all unbounded sectors $S_{d_{1}}$ with bisecting directions $d_{1} \in \mathbb{R}$ and radius $\rho>0$ that obey the requirements of Lemma 4 , for all directions $d_{2} \in \mathbb{R}$, we define the partial map

$$
\begin{aligned}
& \left(u_{1}, u_{2}, z\right) \mapsto U_{d_{1}, d_{2}}\left(u_{1}, u_{2}, z, \epsilon\right) \\
& \quad=\frac{k_{1}}{(2 \pi)^{1 / 2}} \int_{L_{d_{1, u}}} \int_{L_{d_{2}, a}} \int_{-\infty}^{+\infty} \omega_{d_{1}}\left(\tau_{1}, \tau_{2}, m, \epsilon\right) \exp \left(-\left(\frac{\tau_{1}}{u_{1}}\right)^{k_{1}}-\left(\frac{\tau_{2}}{u_{2}}\right)\right) e^{i z m} \frac{d \tau_{1}}{\tau_{1}} \frac{d \tau_{2}}{\tau_{2}} d m
\end{aligned}
$$

for all $\epsilon \in D_{\epsilon_{0}} \backslash\{0\}$, where $\omega_{d_{1}}\left(\tau_{1}, \tau_{2}, m, \epsilon\right)$ is the Borel map built up in Proposition 4 that solves the convolution equation (42), where $\epsilon_{0}>0$ is a small radius chosen in agreement with Proposition 4 and the direction $d_{1, u_{1}} \in \mathbb{R}$ is properly chosen and described below.

The map (92) enjoys the next two features:

- It defines holomorphic function bounded by a constant not relying on $\epsilon$ on the product $U_{1, d_{1}} \times U_{2, d_{2}} \times H_{\beta^{\prime}}$, where $U_{j, d_{j}}$ stand for bounded open sectors centered at 0 with bisecting directions $d_{j}$, for $j=1,2$ and for any given $0<\beta^{\prime}<\beta$,

- it solves the auxiliary problem (30) with prescribed initial data $U_{d_{1}, d_{2}}(0,0, z, \epsilon) \equiv 0$.

Furthermore, the sectors $U_{1, d_{1}}$ and $U_{2, d_{2}}$ are submitted to the next technical constraints:

- There exists a positive real number $\Delta_{1}>0$ such that for all $u_{1} \in U_{1, d_{1}}$, one can select a direction $d_{1, u_{1}} \in \mathbb{R}$ (relying on $u_{1}$ ) such that

$$
\exp \left(\sqrt{-1} d_{1, u_{1}}\right) \in S_{d_{1}} \quad, \quad \cos \left(k_{1}\left(d_{1, u_{1}}-\arg \left(u_{1}\right)\right)>\Delta_{1} .\right.
$$

- The radius $r_{U_{1, d_{1}}}>0$ of $U_{1, d_{1}}$ suffers the next upper bounds

$$
0<r_{U_{1, d_{1}}}<\Delta_{1}^{1 / k_{1}} \frac{|\epsilon|}{\left(\nu+\tilde{\Delta}_{1}\right)^{1 / k_{1}}}
$$

for some positive real number $\tilde{\Delta}_{1}>0$ and where $\Delta_{1}>0$ is defined in the above item. 
- There exists some positive real number $\Delta_{2}>0$ such that

$$
\cos \left(d_{2}-\arg \left(u_{2}\right)\right)>\Delta_{2}
$$

for all $u_{2} \in U_{2, d_{2}}$.

Proof We focus on the first item of the proposition. We consider the map $\omega_{d_{1}}\left(\tau_{1}, \tau_{2}, m, \epsilon\right)$ built up in Proposition 4 and we select two bounded sectors $U_{1, d_{1}}$ and $U_{2, d_{2}}$ fulfilling the above prerequisite (93), (94) and (95). We set $u_{j} \in U_{j, d_{j}}$, for $j=1,2$ and take

$$
\tau_{1}=r_{1} \exp \left(\sqrt{-1} d_{1, u_{1}}\right) \in L_{d_{1, u_{1}}} \quad, \quad \tau_{2}=r_{2} \exp \left(\sqrt{-1} d_{2}\right) \in L_{d_{2}, a}
$$

for given real numbers $r_{1} \geq 0, r_{2} \in[0, a]$. Then, a constant $\varpi>0$ can be found for which the next bounds hold

$$
\begin{aligned}
& \left|\omega_{d_{1}}\left(\tau_{1}, \tau_{2}, m, \epsilon\right)\left\|\exp \left(-\left(\frac{\tau_{1}}{u_{1}}\right)^{k_{1}}-\left(\frac{\tau_{2}}{u_{2}}\right)\right)\right\| e^{i z m}\right| \frac{1}{\left|\tau_{1}\right|\left|\tau_{2}\right|} \\
& \leq \varpi(1+|m|)^{-\mu} e^{-\beta|m|} \frac{1}{|\epsilon|} \exp \left(\nu\left(\frac{r_{1}}{|\epsilon|}\right)^{k_{1}}\right) \exp \left(-\left(\frac{r_{1}}{\left|u_{1}\right|}\right)^{k_{1}} \cos \left(k_{1}\left(d_{1, u_{1}}-\arg \left(u_{1}\right)\right)\right)\right. \\
& \quad \times \exp \left(-\left(\frac{r_{2}}{\left|u_{2}\right|} \cos \left(d_{2}-\arg \left(u_{2}\right)\right)\right) e^{-m \operatorname{Im}(z)}\right. \\
& \leq \varpi(1+|m|)^{-\mu} e^{-\left(\beta-\beta^{\prime}\right)|m|} \frac{1}{|\epsilon|} \exp \left(\nu\left(\frac{r_{1}}{|\epsilon|}\right)^{k_{1}}\right) \exp \left(-\left(\frac{r_{1}}{\left|u_{1}\right|}\right)^{k_{1}} \Delta_{1}\right) \exp \left(-\left(\frac{r_{2}}{\left|u_{2}\right|} \Delta_{2}\right)\right. \\
& \leq \varpi(1+|m|)^{-\mu} e^{-\left(\beta-\beta^{\prime}\right)|m|} \frac{1}{|\epsilon|} \exp \left(-\left(\frac{\tilde{\Delta}_{1}}{|\epsilon|^{k_{1}}} r_{1}^{k_{1}}\right) \exp \left(-\left(\frac{r_{2}}{r_{U_{2, d_{2}}}} \Delta_{2}\right)\right.\right.
\end{aligned}
$$

for all $r_{1} \geq 0, r_{2} \in[0, a]$ and all $m \in \mathbb{R}$, where $r_{U_{2, d_{2}}}>0$ stands for the radius of $U_{2, d_{2}}$. As a result, we reach the next upper bounds

$$
\begin{aligned}
&\left|U_{d_{1}, d_{2}}\left(u_{1}, u_{2}, z, \epsilon\right)\right| \leq \frac{k_{1} \varpi}{(2 \pi)^{1 / 2}} \int_{0}^{+\infty} \frac{1}{|\epsilon|} \exp \left(-\left(\frac{\tilde{\Delta}_{1}}{|\epsilon|^{k_{1}}} r_{1}^{k_{1}}\right) d r_{1}\right. \\
& \times \int_{-\infty}^{+\infty} e^{-\left(\beta-\beta^{\prime}\right)|m|} d m \int_{0}^{a} \exp \left(-\frac{r_{2}}{r_{U_{2, d_{2}}}} \Delta_{2}\right) d r_{2} \\
&=\frac{k_{1} \varpi}{(2 \pi)^{1 / 2}} \int_{0}^{+\infty} \exp \left(-\tilde{\Delta}_{1} r_{2}^{k_{1}}\right) d r_{2} \int_{-\infty}^{+\infty} e^{-\left(\beta-\beta^{\prime}\right)|m|} d m \int_{0}^{a} \exp \left(-\frac{r_{2}}{r_{U_{2, d_{2}}}} \Delta_{2}\right) d r_{2}
\end{aligned}
$$

provided that $u_{j} \in U_{j, d_{j}}, j=1,2, z \in H_{\beta^{\prime}}$ and all $\epsilon \in D_{\epsilon_{0}} \backslash\{0\}$, by the change of variable $r_{2}=r_{1} /|\epsilon|$ in the integral. The right handside of (97) turns out to be a constant unconstrained to $\epsilon$ on $D_{\epsilon_{0}} \backslash\{0\}$. The first item follows.

Concerning the second item, we depart from the Borel map $w_{d_{1}}\left(\tau_{1}, \tau_{2}, m, \epsilon\right)$ which is shown to solve the associated convolution equation (42) in Proposition 4. According to the computations performed in Subsection 3.1, we deduce that for any $\epsilon \in D_{\epsilon_{0}} \backslash\{0\}$, the holomorphic map $U_{d_{1}, d_{2}}\left(u_{1}, u_{2}, z, \epsilon\right)$ given by the expression (92) solves the equations (41), (36), (35) and finally (30) on the domain $U_{1, d_{1}} \times U_{2, d_{2}} \times H_{\beta^{\prime}}$, for prescribed initial data $U_{d_{1}, d_{2}}(0,0, z, \epsilon) \equiv 0$. This yields the second item. 


\section{A finite set of holomorphic solutions to the main initial value problem and their Gevrey asymptotic expansions in power and logarithmic scales}

\subsection{Construction of a finite set of genuine solutions to a properly chosen finite set of related initial value problems}

We first need to refresh the reader's memory of the definition of a good covering in $\mathbb{C}^{*}$ as stated in the reference text book [8], Section XI-2.

Definition 5 Let $\varsigma \geq 2$ be an integer. For all $0 \leq p \leq \varsigma-1$, we set $U_{p}$ as open bounded sectors centered at 0 that share the next three qualities

1. The intersection $U_{p} \cap U_{p+1}$ of two consecutive sectors $U_{p}$ and $U_{p+1}$ is not empty for any $0 \leq p \leq \varsigma-1$, where $U_{\varsigma}=U_{0}$ by convention.

2. The intersection of any three sectors $U_{p} \cap U_{q} \cap U_{r}$ is empty for distinct integers $p, q, r \in$ $\{0, \ldots, \varsigma-1\}$.

3. The union of all the sectors $U_{p}, 0 \leq p \leq \varsigma-1$, covers some punctured neighborhood of 0 in $\mathbb{C}^{*}$, that is

$$
\bigcup_{p=0}^{\varsigma-1} U_{p}=U \backslash\{0\}
$$

for some neighborhood $U$ of 0 in $\mathbb{C}$. Such a set $\underline{\mathcal{U}}=\left\{U_{p}\right\}_{0 \leq p \leq \varsigma-1}$ of sectors is labelled a good covering in $\mathbb{C}^{*}$.

Furthermore, we introduce a notion of fitting finite sets of sectors.

Definition 6 We consider three families of bounded open sectors centered at 0 ,

$$
\underline{\mathcal{U}}_{1}=\left\{U_{1, d_{p}}\right\}_{0 \leq p \leq \varsigma_{1}-1} \quad, \quad \underline{\mathcal{U}}_{2}=\left\{U_{2, \mathfrak{d}_{q}}\right\}_{0 \leq q \leq \varsigma_{2}-1} \quad, \quad \underline{\mathcal{E}}=\left\{\mathcal{E}_{p}\right\}_{0 \leq p \leq \varsigma_{1}-1}
$$

for integers $\varsigma_{j} \geq 2, j=1,2$ and a bounded sector $\mathcal{T}$ centered at 0 that are submitted to the next list of constraints:

1. For all $0 \leq p \leq \varsigma_{1}-1$ and any fixed $\epsilon \in D_{\epsilon_{0}} \backslash\{0\}$, for some given radius $\epsilon_{0}>0$, the sectors $U_{1, d_{p}}$ with bisecting direction $d_{p} \in \mathbb{R}$ is subjected to the next three conditions

- For each direction $d_{p} \in \mathbb{R}, 0 \leq p \leq \varsigma_{1}-1$, one can single out an unbounded sector $S_{d_{p}}$ centered at 0 with bisecting direction $d_{p}$ that satisfies the requirements of Lemma 4 (namely for which the lower bounds (63) and (64) hold)

- For each $0 \leq p \leq \varsigma_{1}-1$, there exists a positive real number $\Delta_{p}>0$ such that for all $u_{1} \in U_{1, d_{p}}$, one can choose a direction $d_{p, u_{1}} \in \mathbb{R}$ (depending on $u_{1}$ ) such that

$$
\exp \left(\sqrt{-1} d_{p, u_{1}}\right) \in S_{d_{p}} \quad, \quad \cos \left(k_{1}\left(d_{p, u_{1}}-\arg \left(u_{1}\right)\right)>\Delta_{p} .\right.
$$

- The radius $r_{U_{1, d_{p}}}>0$ of $U_{1, d_{p}}$ is submitted to the next upper bounds

$$
0<r_{U_{1, d_{p}}}<\Delta_{p}^{1 / k_{1}} \frac{|\epsilon|}{\left(\nu+\tilde{\Delta}_{p}\right)^{1 / k_{1}}}
$$

for some positive real number $\tilde{\Delta}_{p}>0$ and where $\Delta_{p}>0$ is defined in the above item. 
2. The radius $r_{\mathcal{T}}>0$ of the sector $\mathcal{T}$ is subjected to the bounds

$$
r_{\mathcal{T}}<\frac{\Delta_{p}^{1 / k_{1}}}{\left(\nu+\tilde{\Delta}_{p}\right)^{1 / k_{1}}}
$$

where $\Delta_{p}, \tilde{\Delta}_{p}>0$ are defined in $1 .$, for all $0 \leq p \leq \varsigma_{1}-1$. Besides, the sectors $\mathcal{E}_{p}$ share a common radius given by $\epsilon_{0}$, for $0 \leq p \leq \varsigma_{1}-1$.

3. For all $0 \leq p \leq \varsigma_{1}-1$, the sectors $\mathcal{E}_{p}$ and $\mathcal{T}$ satisfy the next feature

$$
\epsilon t \in U_{1, d_{p}}
$$

for all $\epsilon \in \mathcal{E}_{p}$, all $t \in \mathcal{T}$.

4. The set $\mathcal{E}$ represents a good covering in $\mathbb{C}^{*}$. Furthermore, the aperture of the sector $\mathcal{T}$ is taken close enough to 0 in a way that the set

$$
I_{1}=\left\{p \in\left\{0, \ldots, \varsigma_{1}-1\right\} / \epsilon t \notin(-\infty, 0], \text { for all } \epsilon \in \mathcal{E}_{p}, \text { all } t \in \mathcal{T}\right\}
$$

is not empty.

5. For all $0 \leq q \leq \varsigma_{2}-1$, the sectors $U_{2, \mathfrak{d}_{q}}$ with bisecting direction $\mathfrak{d}_{q} \in \mathbb{R}$ obey the next constraint : there exists some positive real number $\check{\Delta}_{q}>0$ such that

$$
\cos \left(\mathfrak{d}_{q}-\arg \left(u_{2}\right)\right)>\check{\Delta}_{q}
$$

for all $u_{2} \in U_{2, \mathfrak{d}_{q}}$. Furthermore, we assume the existence of an index $q_{1} \in\left\{0, \ldots, \varsigma_{2}-1\right\}$ such that $\mathfrak{d}_{q_{1}}=\pi$.

6. The set $\underline{\mathcal{U}}_{2}$ forms a good covering in $\mathbb{C}^{*}$.

We say that the sets sectors $\underline{\mathcal{U}}_{1}, \underline{\mathcal{U}}_{2}, \underline{\mathcal{E}}$ and $\mathcal{T}$ are fitting.

In the coming proposition, we build up a finite family of analytic solutions to auxiliary problems (30) with well chosen forcing terms.

Proposition 6 Consider sets of sectors $\underline{\mathcal{U}}_{1}, \underline{\mathcal{U}}_{2}, \underline{\mathcal{E}}$ and a sector $\mathcal{T}$ that are fitting (in the sense of Definition 6). For each $0 \leq q \leq \varsigma_{2}-1$, the equation

$$
\begin{aligned}
& Q\left(\partial_{z}\right) U_{\mathfrak{d}_{q}}\left(u_{1}, u_{2}, z, \epsilon\right)=u_{1}^{d_{D}}\left(u_{1} \partial_{u_{1}}-u_{2}^{2} \partial_{u_{2}}\right)^{\delta_{D}} R_{D}\left(\partial_{z}\right) U_{\mathfrak{d}_{q}}\left(u_{1}, u_{2}, z, \epsilon\right) \\
& \quad+\sum_{l=1}^{D-1} \epsilon^{\Delta_{l}-d_{l}} u_{1}^{d_{l}}\left(u_{1} \partial_{u_{1}}-u_{2}^{2} \partial_{u_{2}}\right)^{\delta_{l}} a_{l}(z, \epsilon) R_{l}\left(\partial_{z}\right) U_{\mathfrak{d}_{q}}\left(u_{1}, u_{2}, z, \epsilon\right)+F_{\mathfrak{d}_{q}, a}\left(u_{1}, u_{2}, z, \epsilon\right)
\end{aligned}
$$

where the forcing term $F_{\mathfrak{D}_{q}, a}$ is given by the expression (29), possesses a finite set of holomorphic solutions $\left(u_{1}, u_{2}, z\right) \mapsto U_{d_{p}, \mathfrak{d}_{q}}\left(u_{1}, u_{2}, z, \epsilon\right)$, for $0 \leq p \leq \varsigma_{1}-1$, on the domain $U_{1, d_{p}} \times U_{2, \mathfrak{d}_{q}} \times H_{\beta^{\prime}}$, for all $\epsilon \in D_{\epsilon_{0}} \backslash\{0\}$, where $\epsilon_{0}>0$ is taken small enough, for any $0<\beta^{\prime}<\beta$, that fulfills the constraint $U_{d_{p}, \mathfrak{d}_{q}}(0,0, z, \epsilon) \equiv 0$. These maps own the next two important features.

- For all $0 \leq p \leq \varsigma_{1}-1,0 \leq q \leq \varsigma_{2}-1$, the maps $\left(u_{1}, u_{2}, z\right) \mapsto U_{d_{p}, \mathfrak{o}_{q}}\left(u_{1}, u_{2}, z, \epsilon\right)$ are bounded by a constant, that is independent of $\epsilon$ on $D_{\epsilon_{0}} \backslash\{0\}$, on the product $U_{1, d_{p}} \times U_{2, \mathfrak{d}_{q}} \times H_{\beta^{\prime}}$. 
- For all $0 \leq p \leq \varsigma_{1}-1,0 \leq q \leq \varsigma_{2}-1$, the maps $U_{d_{p}, \mathfrak{d}_{q}}$ are represented as Fourier inverse and Laplace transforms,

$$
\begin{aligned}
& U_{d_{p}, \mathfrak{d}_{q}}\left(u_{1}, u_{2}, z, \epsilon\right) \\
= & \frac{k_{1}}{(2 \pi)^{1 / 2}} \int_{L_{d_{p, u_{1}}}} \int_{L_{d_{\mathfrak{d}_{q}, a}}} \int_{-\infty}^{+\infty} \omega_{d_{p}}\left(\tau_{1}, \tau_{2}, m, \epsilon\right) \exp \left(-\left(\frac{\tau_{1}}{u_{1}}\right)^{k_{1}}-\left(\frac{\tau_{2}}{u_{2}}\right)\right) e^{i z m} \frac{d \tau_{1}}{\tau_{1}} \frac{d \tau_{2}}{\tau_{2}} d m
\end{aligned}
$$

where the Borel maps $\left(\tau_{1}, \tau_{2}, m\right) \mapsto \omega_{d_{p}}\left(\tau_{1}, \tau_{2}, m, \epsilon\right)$ belong to the Banach space $F_{\left(\nu, \beta, \mu, k_{1}, \rho, a, \epsilon\right)}^{d_{p}}$ with bounds

$$
\sup _{\epsilon \in D_{\epsilon_{0}} \backslash\{0\}}\left\|\omega_{d_{p}}\left(\tau_{1}, \tau_{2}, m, \epsilon\right)\right\|_{\left(\nu, \beta, \mu, k_{1}, \rho, a, \epsilon\right)} \leq \varpi_{p}
$$

for some well chosen constants $\varpi_{p}>0$ and radius $\rho>0$, for all $\epsilon \in D_{\epsilon_{0}} \backslash\{0\}$.

Proof This proposition is a straight consequence of Proposition 5 and the definition of the fitting sectors chosen overhead in the proposition.

In the next proposition we study a finite set of maps related to the analytic solutions (105) to the problems (104) stated in Proposition 6. In particular we are interested in the control of their consecutive differences which turns out to be a crucial information for reaching their asymptotic features (see Subsection 4.2).

Proposition 7 We prescribe a sets of sectors $\underline{\mathcal{U}}_{1}, \underline{\mathcal{U}}_{2}, \underline{\mathcal{E}}$ and a sector $\mathcal{T}$ that are fitting (in the sense of Definition 6). For each $0 \leq p \leq \varsigma_{1}-1,0 \leq q \leq \varsigma_{2}-1$, we introduce the map

$$
\mathbb{U}_{d_{p}, \mathfrak{d}_{q}}\left(t, u_{2}, z, \epsilon\right):=U_{d_{p}, \mathfrak{d}_{q}}\left(\epsilon t, u_{2}, z, \epsilon\right)
$$

where $U_{d_{p}, \mathrm{o}_{q}}$ is built up in Proposition 6. The next properties hold.

- For all $0 \leq p \leq \varsigma_{1}-1,0 \leq q \leq \varsigma_{2}-1$, the maps $\mathbb{U}_{d_{p}, \mathfrak{d}_{q}}\left(t, u_{2}, z, \epsilon\right)$ are bounded holomorphic on the product $\mathcal{T} \times U_{2, \mathfrak{o}_{q}} \times H_{\beta^{\prime}} \times \mathcal{E}_{p}$.

- Let $q=q_{0} \in\left\{0, \ldots, \varsigma_{2}-1\right\}$ be a given integer. For all $0 \leq p \leq \varsigma_{1}-1$, one can find two constants $M_{p, 1}, K_{p, 1}>0$ such that

$$
\left|\mathbb{U}_{d_{p+1}, \mathfrak{d}_{q_{0}}}\left(t, u_{2}, z, \epsilon\right)-\mathbb{U}_{d_{p}, \mathfrak{d}_{q_{0}}}\left(t, u_{2}, z, \epsilon\right)\right| \leq M_{p, 1} \exp \left(-\frac{K_{p, 1}}{|\epsilon|^{k_{1}}}\right)
$$

for all $t \in \mathcal{T}$, all $\epsilon \in \mathcal{E}_{p+1} \cap \mathcal{E}_{p}$, all $u_{2} \in U_{2, \mathrm{~d}_{q_{0}}}$, all $z \in H_{\beta^{\prime}}$, where by convention $d_{\varsigma_{1}}=d_{0}$.

- Let $p=p_{0} \in\left\{0, \ldots, \varsigma_{1}-1\right\}$ be a prescribed integer. For any $0 \leq q \leq \varsigma_{2}-1$, two constants $M_{q, 2}, K_{q, 2}>0$ can be singled out for which

$$
\left|\mathbb{U}_{d_{p_{0}, \mathfrak{d}_{q+1}}}\left(t, u_{2}, z, \epsilon\right)-\mathbb{U}_{d_{p_{0}, \mathfrak{d}_{q}}}\left(t, u_{2}, z, \epsilon\right)\right| \leq M_{q, 2} \exp \left(-\frac{K_{q, 2}}{\left|u_{2}\right|}\right)
$$

holds, provided that $t \in \mathcal{T}, \epsilon \in \mathcal{E}_{p_{0}}$, all $u_{2} \in U_{2, \mathfrak{d}_{q+1}} \cap U_{2, \mathrm{o}_{q}}$, all $z \in H_{\beta^{\prime}}$, where the convention $\mathfrak{d}_{\varsigma_{2}}=\mathfrak{d}_{0}$ is taken. 
Proof The first item follows directly from the properties of the maps $U_{d_{p}, \mathrm{~d}_{q}}$ stated in Proposition 6 and from the features of the sectors $\mathcal{E}_{p}$ and $\mathcal{T}$ listed in the points 2. and 3. of Definition 6.

The second item needs more effort and uses a path deformation argument. Let us fix $q=$ $q_{0} \in\left\{0, \ldots, \varsigma_{2}-1\right\}$ and take $p \in\left\{0, \ldots, \varsigma_{1}-1\right\}$. For any given $\tau_{2} \in D_{a}, m \in \mathbb{R}, \epsilon \in D_{\epsilon_{0}} \backslash\{0\}$, the partial maps $\tau_{1} \mapsto \omega_{d_{j}}\left(\tau_{1}, \tau_{2}, m, \epsilon\right), j=p, p+1$, are analytic continuation on the sector $S_{d_{j}}$ of a common analytic map denoted $\tau_{1} \mapsto \omega\left(\tau_{1}, \tau_{2}, m, \epsilon\right)$ on the disc $D_{\rho}$.

For any fixed $\epsilon \in \mathcal{E}_{p+1} \cap \mathcal{E}_{p}$ and $t \in \mathcal{T}$, we deform the oriented path $L_{d_{p+1, \epsilon t}}-L_{d_{p}, \epsilon t}$ into the union of three oriented pieces

- Two halflines

$$
L_{d_{p+1, \epsilon t} ; \rho / 2}=[\rho / 2,+\infty) \exp \left(\sqrt{-1} d_{p+1, \epsilon t}\right) \quad, \quad-L_{d_{p, \epsilon t} ; \rho / 2}=-[\rho / 2,+\infty) \exp \left(\sqrt{-1} d_{p, \epsilon t}\right) .
$$

- An arc of circle centered at 0 with radius $\rho / 2$ that rely the above two halflines

$$
C_{p, p+1, \epsilon t ; \rho / 2}=\left\{\frac{\rho}{2} e^{\sqrt{-1} \theta} / \theta \in\left(d_{p, \epsilon t}, d_{p+1, \epsilon t}\right)\right\} .
$$

By means of the classical Cauchy's theorem, we recast the next difference as a sum of three terms

$$
\begin{aligned}
& \mathbb{U}_{d_{p+1}, \mathfrak{o}_{q_{0}}}\left(t, u_{2}, z, \epsilon\right)-\mathbb{U}_{d_{p}, \mathfrak{o}_{0}}\left(t, u_{2}, z, \epsilon\right) \\
= & \frac{k_{1}}{(2 \pi)^{1 / 2}} \int_{L_{d_{p+1, \epsilon t}, \rho / 2}} \int_{L_{\mathrm{o}_{q_{0}}, a}} \int_{-\infty}^{+\infty} \omega_{d_{p+1}}\left(\tau_{1}, \tau_{2}, m, \epsilon\right) \exp \left(-\left(\frac{\tau_{1}}{\epsilon t}\right)^{k_{1}}-\left(\frac{\tau_{2}}{u_{2}}\right)\right) e^{i z m} \frac{d \tau_{1}}{\tau_{1}} \frac{d \tau_{2}}{\tau_{2}} d m \\
- & \frac{k_{1}}{(2 \pi)^{1 / 2}} \int_{L_{d_{p, \epsilon t}, \rho / 2}} \int_{L_{\mathrm{o}_{q_{0}}, a}} \int_{-\infty}^{+\infty} \omega_{d_{p}}\left(\tau_{1}, \tau_{2}, m, \epsilon\right) \exp \left(-\left(\frac{\tau_{1}}{\epsilon t}\right)^{k_{1}}-\left(\frac{\tau_{2}}{u_{2}}\right)\right) e^{i z m} \frac{d \tau_{1}}{\tau_{1}} \frac{d \tau_{2}}{\tau_{2}} d m \\
& +\frac{k_{1}}{(2 \pi)^{1 / 2}} \int_{C_{p, p+1, \epsilon t ; \rho / 2}} \int_{L_{\mathfrak{D}_{0}, a}} \int_{-\infty}^{+\infty} \omega\left(\tau_{1}, \tau_{2}, m, \epsilon\right) \exp \left(-\left(\frac{\tau_{1}}{\epsilon t}\right)^{k_{1}}-\left(\frac{\tau_{2}}{u_{2}}\right)\right) e^{i z m} \frac{d \tau_{1}}{\tau_{1}} \frac{d \tau_{2}}{\tau_{2}} d m
\end{aligned}
$$

Upper bounds are provided for the first building block of (110),

$$
I_{1}=\left|\frac{k_{1}}{(2 \pi)^{1 / 2}} \int_{L_{d_{p+1, \epsilon t}, \rho / 2}} \int_{L_{\mathfrak{d}_{0}, a}} \int_{-\infty}^{+\infty} \omega_{d_{p+1}}\left(\tau_{1}, \tau_{2}, m, \epsilon\right) \exp \left(-\left(\frac{\tau_{1}}{\epsilon t}\right)^{k_{1}}-\left(\frac{\tau_{2}}{u_{2}}\right)\right) e^{i z m} \frac{d \tau_{1}}{\tau_{1}} \frac{d \tau_{2}}{\tau_{2}} d m\right| .
$$

Based on the bounds (96) and (106) together with the requirements described in Definition 6, we arrive at

$$
\begin{aligned}
& \text { (111) } I_{1} \leq \frac{\varpi_{p+1} k_{1}}{(2 \pi)^{1 / 2}}\left(\int_{0}^{a} \exp \left(-\left(\frac{r_{2}}{\left|u_{2}\right|}\right) \check{\Delta}_{q_{0}}\right) d r_{2}\right)\left(\int_{-\infty}^{+\infty} e^{-\left(\beta-\beta^{\prime}\right)|m|} d m\right) \\
& \times\left(\int_{\rho / 2}^{+\infty} \frac{1}{|\epsilon|} \exp \left(-\frac{\tilde{\Delta}_{p+1}}{|\epsilon|^{k_{1}}} r_{1}^{k_{1}}\right) d r_{1}\right) \leq \frac{\varpi_{p+1} k_{1}}{(2 \pi)^{1 / 2}}\left[-\frac{\left|u_{2}\right|}{\check{\Delta}_{q_{0}}} \exp \left(-\frac{\check{\Delta}_{q_{0}}}{\left|u_{2}\right|} r_{2}\right)\right]_{0}^{a} \times 2 \int_{0}^{+\infty} e^{-\left(\beta-\beta^{\prime}\right) m} d m \\
& \times \int_{\rho / 2}^{+\infty} \frac{1}{|\epsilon|}\left\{\frac{|\epsilon|^{k_{1}}}{\tilde{\Delta}_{p+1}} \frac{1}{k_{1} r_{1}^{k_{1}-1}}\right\}\left(\frac{\tilde{\Delta}_{p+1}}{|\epsilon|^{k_{1}}} k_{1} r_{1}^{k_{1}-1} \exp \left(-\frac{\tilde{\Delta}_{p+1}}{|\epsilon|^{k_{1}}} r_{1}^{k_{1}}\right)\right) d r_{1} \\
& \leq \frac{\varpi_{p+1} k_{1}}{(2 \pi)^{1 / 2}} \frac{\left|u_{2}\right|}{\check{\Delta}_{q_{0}}}\left(1-\exp \left(-\frac{\check{\Delta}_{q_{0}}}{\left|u_{2}\right|} a\right)\right) \frac{2}{\beta-\beta^{\prime}} \frac{|\epsilon|^{k_{1}-1}}{\tilde{\Delta}_{p+1}} \frac{1}{k_{1}(\rho / 2)^{k_{1}-1}}\left[-\exp \left(-\frac{\tilde{\Delta}_{p+1}}{|\epsilon|^{k_{1}}} r_{1}^{k_{1}}\right)\right]_{\rho / 2}^{+\infty} \\
& \leq \frac{\varpi_{p+1} k_{1}}{(2 \pi)^{1 / 2}} \frac{\left|u_{2}\right|}{\check{\Delta}_{q_{0}}} \frac{2}{\beta-\beta^{\prime}} \frac{|\epsilon|^{k_{1}-1}}{\tilde{\Delta}_{p+1}} \frac{1}{k_{1}(\rho / 2)^{k_{1}-1}} \exp \left(-\frac{\tilde{\Delta}_{p+1}}{|\epsilon|^{k_{1}}}(\rho / 2)^{k_{1}}\right)
\end{aligned}
$$


provided that $\epsilon \in \mathcal{E}_{p+1} \cap \mathcal{E}_{p}, t \in \mathcal{T}, u_{2} \in U_{2, \mathfrak{d}_{q_{0}}}$ and $z \in H_{\beta^{\prime}}$

In a similar manner, we can exhibit explicit upper bounds for the second constituent of (110),

$$
I_{2}=\left|\frac{k_{1}}{(2 \pi)^{1 / 2}} \int_{L_{d_{p, \epsilon t}, \rho / 2}} \int_{L_{\mathrm{o}_{0}, a}} \int_{-\infty}^{+\infty} \omega_{d_{p}}\left(\tau_{1}, \tau_{2}, m, \epsilon\right) \exp \left(-\left(\frac{\tau_{1}}{\epsilon t}\right)^{k_{1}}-\left(\frac{\tau_{2}}{u_{2}}\right)\right) e^{i z m} \frac{d \tau_{1}}{\tau_{1}} \frac{d \tau_{2}}{\tau_{2}} d m\right|
$$

in the form

$$
I_{2} \leq \frac{\varpi_{p} k_{1}}{(2 \pi)^{1 / 2}} \frac{\left|u_{2}\right|}{\check{\Delta}_{q_{0}}} \frac{2}{\beta-\beta^{\prime}} \frac{|\epsilon|^{k_{1}-1}}{\tilde{\Delta}_{p}} \frac{1}{k_{1}(\rho / 2)^{k_{1}-1}} \exp \left(-\frac{\tilde{\Delta}_{p}}{|\epsilon|^{k_{1}}}(\rho / 2)^{k_{1}}\right)
$$

for all $\epsilon \in \mathcal{E}_{p+1} \cap \mathcal{E}_{p}, t \in \mathcal{T}, u_{2} \in U_{2, \mathfrak{o}_{q_{0}}}$ and $z \in H_{\beta^{\prime}}$.

At last, we control the integral along the arc of circle appearing in (110)

$$
\begin{aligned}
& I_{3}= \\
& \left|\frac{k_{1}}{(2 \pi)^{1 / 2}} \int_{C_{p, p+1, \epsilon t ; / 2}} \int_{L_{\mathrm{D}_{0}, a}} \int_{-\infty}^{+\infty} \omega\left(\tau_{1}, \tau_{2}, m, \epsilon\right) \exp \left(-\left(\frac{\tau_{1}}{\epsilon t}\right)^{k_{1}}-\left(\frac{\tau_{2}}{u_{2}}\right)\right) e^{i z m} \frac{d \tau_{1}}{\tau_{1}} \frac{d \tau_{2}}{\tau_{2}} d m\right| .
\end{aligned}
$$

As a consequence of the bounds (106), we get that

$$
\left|\omega\left(\tau_{1}, \tau_{2}, m, \epsilon\right)\right| \leq \sup \left(\varpi_{p}, \varpi_{p+1}\right)(1+|m|)^{-\mu} e^{-\beta|m|} \frac{\rho / 2}{|\epsilon|}\left|\tau_{2}\right| \exp \left(\nu \frac{(\rho / 2)^{k_{1}}}{|\epsilon|^{k_{1}}}\right)
$$

for all $\tau_{1} \in C_{p, p+1, \epsilon t ; \rho / 2}, \tau_{2} \in L_{\mathfrak{D}_{q_{0}}, a}, m \in \mathbb{R}$ and $\epsilon \in \mathcal{E}_{p+1} \cap \mathcal{E}_{p}$. Furthermore, in view of the restrictions displayed in Definition 6, it turns out that

$$
\cos \left(k_{1}(\theta-\arg (\epsilon t))\right)>\Delta_{p, p+1}:=\min \left(\Delta_{p}, \Delta_{p+1}\right)
$$

for all $t \in \mathcal{T}, \epsilon \in \mathcal{E}_{p} \cap \mathcal{E}_{p+1}$, provided that the angle $\theta$ belongs to $\left(d_{p, \epsilon t}, d_{p+1, \epsilon t}\right)$ or $\left(d_{p+1, \epsilon t}, d_{p, \epsilon t}\right)$. Hence, by virtue of (114) and (115), we come up with some constant $\tilde{\Delta}_{p, p+1}>0$ with

$$
\begin{aligned}
& I_{3} \leq \frac{k_{1}}{(2 \pi)^{1 / 2}} \sup \left(\varpi_{p}, \varpi_{p+1}\right)\left(\int_{0}^{a} \exp \left(-\left(\frac{r_{2}}{\left|u_{2}\right|}\right) \check{\Delta}_{q_{0}}\right) d r_{2}\right)\left(\int_{-\infty}^{+\infty} e^{-\left(\beta-\beta^{\prime}\right)|m|} d m\right) \\
& \quad \times\left|\int_{d_{p, \epsilon t}}^{d_{p+1, \epsilon t}} \frac{1}{|\epsilon|} \exp \left(\nu \frac{(\rho / 2)^{k_{1}}}{|\epsilon|^{k_{1}}}\right) \exp \left(-\frac{(\rho / 2)^{k_{1}}}{|\epsilon t|^{k_{1}}} \Delta_{p, p+1}\right) \frac{\rho}{2} d \theta\right| \\
& \leq \frac{k_{1}}{(2 \pi)^{1 / 2}} \sup \left(\varpi_{p}, \varpi_{p+1}\right) \frac{\left|u_{2}\right|}{\check{\Delta}_{q_{0}}} \frac{2}{\beta-\beta^{\prime}}\left|d_{p+1, \epsilon t}-d_{p, \epsilon t}\right|\left|\frac{1}{\epsilon}\right| \exp \left(-\frac{\tilde{\Delta}_{p, p+1}}{|\epsilon|^{k_{1}}}(\rho / 2)^{k_{1}}\right) \frac{\rho}{2}
\end{aligned}
$$

for all $\epsilon \in \mathcal{E}_{p+1} \cap \mathcal{E}_{p}, t \in \mathcal{T}, u_{2} \in U_{2, \mathfrak{o}_{q_{0}}}$ and $z \in H_{\beta^{\prime}}$. Besides, based on (116) and bearing in mind the classical bounds

$$
\sup _{x \geq 0} x^{m_{1}} e^{-m_{2} x}=\left(\frac{m_{1}}{m_{2}}\right)^{m_{1}} e^{-m_{1}}
$$

for any real numbers $m_{1} \geq 0, m_{2}>0$, the next bounds

$$
\begin{aligned}
& I_{3} \leq \frac{k_{1}}{(2 \pi)^{1 / 2}} \sup \left(\varpi_{p}, \varpi_{p+1}\right) \frac{\left|u_{2}\right|}{\check{\Delta}_{q_{0}}} \frac{2}{\beta-\beta^{\prime}}\left|d_{p+1, \epsilon t}-d_{p, \epsilon t}\right| \frac{\rho}{2}\left|\frac{1}{\epsilon}\right| \exp \left(-\frac{\tilde{\Delta}_{p, p+1}}{2|\epsilon|^{k_{1}}}(\rho / 2)^{k_{1}}\right) \\
& \quad \times \exp \left(-\frac{\tilde{\Delta}_{p, p+1}}{2|\epsilon|^{k_{1}}}(\rho / 2)^{k_{1}}\right) \\
& \leq \frac{k_{1}}{(2 \pi)^{1 / 2}} \sup \left(\varpi_{p}, \varpi_{p+1}\right) \frac{\left|u_{2}\right|}{\check{\Delta}_{q_{0}}} \frac{2}{\beta-\beta^{\prime}}\left|d_{p+1, \epsilon t}-d_{p, \epsilon t}\right| \frac{\rho}{2} \mathcal{C}_{k_{1}, \rho, \tilde{\Delta}_{p, p+1}} \exp \left(-\frac{\tilde{\Delta}_{p, p+1}}{2|\epsilon|^{k_{1}}}(\rho / 2)^{k_{1}}\right)
\end{aligned}
$$


where

$$
\mathcal{C}_{k_{1}, \rho, \tilde{\Delta}_{p, p+1}}=\sup _{x \geq 0} x^{1 / k_{1}} \exp \left(-\frac{\tilde{\Delta}_{p, p+1}}{2}(\rho / 2)^{k_{1}} x\right)=\left(\frac{1 / k_{1}}{\tilde{\Delta}_{p, p+1}(\rho / 2)^{k_{1}} / 2}\right)^{1 / k_{1}} e^{-1 / k_{1}}
$$

provided that $\epsilon \in \mathcal{E}_{p+1} \cap \mathcal{E}_{p}, t \in \mathcal{T}, u_{2} \in U_{2, \mathfrak{o}_{0}}$ and $z \in H_{\beta^{\prime}}$. In summary, the splitting (110) along with the bounds (111), (112), (117) breed the awaited estimates (108).

The third item leads to comparable bounds as the ones reached in the second item and leans again on a path deformation argument. Indeed, we set $p_{0} \in\left\{0, \ldots, \varsigma_{1}-1\right\}$ and take $q \in\left\{0, \ldots, \varsigma_{2}-1\right\}$. For any prescribed $\tau_{1} \in S_{d_{p_{0}}}, m \in \mathbb{R}$ and $\epsilon \in \mathcal{E}_{p_{0}}$, the partial map $\tau_{2} \mapsto \omega_{d_{p_{0}}}\left(\tau_{1}, \tau_{2}, m, \epsilon\right)$ is analytic on the disc $D_{a}$. As a result, we may bend the oriented path $L_{\mathfrak{d}_{q+1}, a}-L_{\mathfrak{d}_{q}, a}$ into the union of three oriented basic geometrical loci

- two segments

$$
L_{\mathfrak{d}_{q+1}, a, a / 2}=[a / 2, a] \exp \left(\sqrt{-1} \mathfrak{d}_{q+1}\right) \quad, \quad-L_{\mathfrak{d}_{q}, a, a / 2}=-[a / 2, a] \exp \left(\sqrt{-1} \mathfrak{d}_{q}\right) .
$$

- An arc of circle centered at 0 with radius $a / 2$,

$$
C_{q, q+1, a / 2}=\left\{\frac{a}{2} e^{\sqrt{-1} \theta} / \theta \in\left[\mathfrak{d}_{q}, \mathfrak{d}_{q+1}\right]\right\}
$$

joining the above two segments.

By dint of the classical Cauchy's theorem, we can reorganize the following difference as a sum of three contributions

$$
\text { 8) } \begin{aligned}
& \mathbb{U}_{d_{p_{0}, \mathfrak{o}_{q+1}}}\left(t, u_{2}, z, \epsilon\right)-\mathbb{U}_{d_{p_{0}}, \mathfrak{o}_{q}}\left(t, u_{2}, z, \epsilon\right) \\
= & \frac{k_{1}}{(2 \pi)^{1 / 2}} \int_{L_{d_{p_{0}, \epsilon t}}} \int_{L_{\mathfrak{o}_{q+1}, a, a / 2}} \int_{-\infty}^{+\infty} \omega_{d_{p_{0}}}\left(\tau_{1}, \tau_{2}, m, \epsilon\right) \exp \left(-\left(\frac{\tau_{1}}{\epsilon t}\right)^{k_{1}}-\left(\frac{\tau_{2}}{u_{2}}\right)\right) e^{i z m} \frac{d \tau_{1}}{\tau_{1}} \frac{d \tau_{2}}{\tau_{2}} d m \\
- & \frac{k_{1}}{(2 \pi)^{1 / 2}} \int_{L_{d_{p_{0}, \epsilon t}}} \int_{L_{\mathfrak{o}_{q}, a, a / 2}} \int_{-\infty}^{+\infty} \omega_{d_{p_{0}}}\left(\tau_{1}, \tau_{2}, m, \epsilon\right) \exp \left(-\left(\frac{\tau_{1}}{\epsilon t}\right)^{k_{1}}-\left(\frac{\tau_{2}}{u_{2}}\right)\right) e^{i z m} \frac{d \tau_{1}}{\tau_{1}} \frac{d \tau_{2}}{\tau_{2}} d m \\
& +\frac{k_{1}}{(2 \pi)^{1 / 2}} \int_{L_{d_{p_{0}}, \epsilon t}} \int_{C_{q, q+1, a / 2}} \int_{-\infty}^{+\infty} \omega_{d_{p_{0}}}\left(\tau_{1}, \tau_{2}, m, \epsilon\right) \exp \left(-\left(\frac{\tau_{1}}{\epsilon t}\right)^{k_{1}}-\left(\frac{\tau_{2}}{u_{2}}\right)\right) e^{i z m} \frac{d \tau_{1}}{\tau_{1}} \frac{d \tau_{2}}{\tau_{2}} d m
\end{aligned}
$$

We plan to upper bound the first part of (118), namely

$$
=\left|\frac{k_{1}}{(2 \pi)^{1 / 2}} \int_{L_{d_{p_{0}, \epsilon t}}} \int_{L_{\mathrm{o}_{q+1}, a, a / 2}} \int_{-\infty}^{+\infty} \omega_{d_{p_{0}}}\left(\tau_{1}, \tau_{2}, m, \epsilon\right) \exp \left(-\left(\frac{\tau_{1}}{\epsilon t}\right)^{k_{1}}-\left(\frac{\tau_{2}}{u_{2}}\right)\right) e^{i z m} \frac{d \tau_{1}}{\tau_{1}} \frac{d \tau_{2}}{\tau_{2}} d m\right|
$$

Drew on the bounds (96) and (106) along with the requirements described in Definition 6 and 
by means of the change of variable $s_{1}=r_{1} /|\epsilon|$ in the integral, we deduce that

$$
\begin{gathered}
J_{1} \leq \frac{\varpi_{p_{0}} k_{1}}{(2 \pi)^{1 / 2}}\left(\int_{a / 2}^{a} \exp \left(-\left(\frac{r_{2}}{\left|u_{2}\right|} \check{\Delta}_{q+1}\right) d r_{2}\right)\left(\int_{-\infty}^{+\infty} e^{-\left(\beta-\beta^{\prime}\right)|m|} d m\right)\right. \\
\times\left(\int_{0}^{+\infty} \frac{1}{|\epsilon|} \exp \left(-\frac{\tilde{\Delta}_{p_{0}}}{|\epsilon|^{k_{1}}} r_{1}^{k_{1}}\right) d r_{1}\right) \\
\leq \frac{\varpi_{p_{0}} k_{1}}{(2 \pi)^{1 / 2}} \int_{0}^{+\infty} \exp \left(-\tilde{\Delta}_{p_{0}} s_{1}^{k_{1}}\right) d s_{1} \frac{2}{\beta-\beta^{\prime}} \frac{\left|u_{2}\right|}{\check{\Delta}_{q+1}}\left(\exp \left(-\frac{\check{\Delta}_{q+1}}{\left|u_{2}\right|} \frac{a}{2}\right)-\exp \left(-\frac{\check{\Delta}_{q+1}}{\left|u_{2}\right|} a\right)\right) \\
\leq \frac{\varpi_{p_{0}} k_{1}}{(2 \pi)^{1 / 2}} \int_{0}^{+\infty} \exp \left(-\tilde{\Delta}_{p_{0}} s_{1}^{k_{1}}\right) d s_{1} \frac{2}{\beta-\beta^{\prime}} \frac{\left|u_{2}\right|}{\check{\Delta}_{q+1}}\left(1-\exp \left(-\frac{\check{\Delta}_{q+1}}{\left|u_{2}\right|} \frac{a}{2}\right)\right) \exp \left(-\frac{\check{\Delta}_{q+1}}{\left|u_{2}\right|} \frac{a}{2}\right) \\
\leq \frac{\varpi_{p_{0}} k_{1}}{(2 \pi)^{1 / 2}} \int_{0}^{+\infty} \exp \left(-\tilde{\Delta}_{p_{0}} s_{1}^{k_{1}}\right) d s_{1} \frac{2}{\beta-\beta^{\prime}} \frac{\left|u_{2}\right|}{\check{\Delta}_{q+1}} \exp \left(-\frac{\check{\Delta}_{q+1}}{\left|u_{2}\right|} \frac{a}{2}\right)
\end{gathered}
$$

provided that $\epsilon \in \mathcal{E}_{p_{0}}, t \in \mathcal{T}, u_{2} \in U_{2, \mathfrak{d}_{q+1}} \cap U_{2, \mathfrak{o}_{q}}$ and $z \in H_{\beta^{\prime}}$.

Much the same as above, the second piece of the decomposition (118),

$$
\begin{aligned}
& J_{2} \\
& =\left|\frac{k_{1}}{(2 \pi)^{1 / 2}} \int_{L_{d_{p_{0}, \epsilon t}}} \int_{L_{\mathrm{o}_{q}, a, a / 2}} \int_{-\infty}^{+\infty} \omega_{d_{p_{0}}}\left(\tau_{1}, \tau_{2}, m, \epsilon\right) \exp \left(-\left(\frac{\tau_{1}}{\epsilon t}\right)^{k_{1}}-\left(\frac{\tau_{2}}{u_{2}}\right)\right) e^{i z m} \frac{d \tau_{1}}{\tau_{1}} \frac{d \tau_{2}}{\tau_{2}} d m\right|
\end{aligned}
$$

can be upper controled as

$$
J_{2} \leq \frac{\varpi_{p_{0}} k_{1}}{(2 \pi)^{1 / 2}} \int_{0}^{+\infty} \exp \left(-\tilde{\Delta}_{p_{0}} s_{1}^{k_{1}}\right) d s_{1} \frac{2}{\beta-\beta^{\prime}} \frac{\left|u_{2}\right|}{\check{\Delta}_{q}} \exp \left(-\frac{\check{\Delta}_{q}}{\left|u_{2}\right|} \frac{a}{2}\right)
$$

as long as $\epsilon \in \mathcal{E}_{p_{0}}, t \in \mathcal{T}, u_{2} \in U_{2, \mathfrak{d}_{q+1}} \cap U_{2, \mathfrak{d}_{q}}$ and $z \in H_{\beta^{\prime}}$.

The closing block of (118),

$$
=\left|\frac{k_{1}}{(2 \pi)^{1 / 2}} \int_{L_{d_{p_{0}, \epsilon t}}} \int_{C_{q, q+1, a / 2}} \int_{-\infty}^{+\infty} \omega_{d_{p_{0}}}\left(\tau_{1}, \tau_{2}, m, \epsilon\right) \exp \left(-\left(\frac{\tau_{1}}{\epsilon t}\right)^{k_{1}}-\left(\frac{\tau_{2}}{u_{2}}\right)\right) e^{i z m} \frac{d \tau_{1}}{\tau_{1}} \frac{d \tau_{2}}{\tau_{2}} d m\right|
$$

can be measured as follows. Owing to the lower bounds (103) set up in Definition 6, we check that

$$
\cos \left(\theta-\arg \left(u_{2}\right)\right)>\check{\Delta}_{q, q+1}:=\min \left(\check{\Delta}_{q}, \check{\Delta}_{q+1}\right)
$$

provided that $u_{2} \in U_{2, \mathfrak{d}_{q+1}} \cap U_{2, \mathfrak{d}_{q}}$, whenever the angle $\theta$ belongs to $\left(\mathfrak{d}_{q}, \mathfrak{d}_{q+1}\right)$ or $\left(\mathfrak{d}_{q+1}, \mathfrak{d}_{q}\right)$. On the basis of the bounds (96), (106) and (124) together with the requirements stemming from Definition 6 and by means of the change of variable $s_{1}=r_{1} /|\epsilon|$ in the integral, we reach

$$
\begin{aligned}
J_{3} \leq \frac{\varpi_{p_{0}} k_{1}}{(2 \pi)^{1 / 2}}\left(\int_{-\infty}^{+\infty} e^{-\left(\beta-\beta^{\prime}\right)|m|} d m\right)\left(\int_{0}^{+\infty} \frac{1}{|\epsilon|} \exp \left(-\frac{\tilde{\Delta}_{p_{0}}}{|\epsilon|^{k_{1}}} r_{1}^{k_{1}}\right) d r_{1}\right) \\
\quad \times\left|\int_{\mathfrak{d}_{q}}^{\mathfrak{d}_{q+1}} \exp \left(-\frac{a / 2}{\left|u_{2}\right|} \check{\Delta}_{q, q+1}\right) \frac{a}{2} d \theta\right| \\
\leq \frac{\varpi_{p_{0}} k_{1}}{(2 \pi)^{1 / 2}} \int_{0}^{+\infty} \exp \left(-\tilde{\Delta}_{p_{0}} s_{1}^{k_{1}}\right) d s_{1} \frac{2}{\beta-\beta^{\prime}}\left|\mathfrak{d}_{q+1}-\mathfrak{d}_{q}\right| \frac{a}{2} \exp \left(-\frac{a / 2}{\left|u_{2}\right|} \check{\Delta}_{q, q+1}\right)
\end{aligned}
$$

for all $\epsilon \in \mathcal{E}_{p_{0}}, t \in \mathcal{T}, u_{2} \in U_{2, \mathfrak{d}_{q+1}} \cap U_{2, \mathfrak{o}_{q}}$ and $z \in H_{\beta^{\prime}}$.

Eventually, the bounds (120), (122) and (125) reached above for the quantities $J_{1}, J_{2}$ and $J_{3}$ applied to the splitting of the difference (118) beget the forecast bounds (109). 


\subsection{Gevrey asymptotic expansions for the related maps to the analytic solu- tions of (104).}

We first remind the reader a result known as the Ramis-Sibuya theorem in the literature, see Lemma XI-2-6 in [8]. It represents a prominent tool in the proof of our main result stated in the next subsection.

Theorem (R.S.) Let $\left(\mathbb{F},\|.\|_{\mathbb{F}}\right)$ be a Banach space over $\mathbb{C}$ and we consider a good covering $\left\{U_{p}\right\}_{0 \leq p \leq \varsigma-1}$ in $\mathbb{C}^{*}$ as described in Definition 5. For all $0 \leq p \leq \varsigma-1$, we set $G_{p}: U_{p} \rightarrow \mathbb{F}$ as holomorphic functions that are subjected to the next two constraints

1. The maps $G_{p}$ are bounded on $U_{p}$ for all $0 \leq p \leq \varsigma-1$.

2. The difference $\Theta_{p}(u)=G_{p+1}(u)-G_{p}(u)$ defines a holomorphic map on the intersection $Z_{p}=U_{p+1} \cap U_{p}$ which is exponentially flat of order $k$, for some integer $k \geq 1$, meaning that one can select two constants $C_{p}, A_{p}>0$ for which

$$
\left\|\Theta_{p}(u)\right\|_{\mathbb{F}} \leq C_{p} \exp \left(-\frac{A_{p}}{|u|^{k}}\right)
$$

holds provided that $u \in Z_{p}$, for all $0 \leq p \leq \varsigma-1$. By convention, we set $G_{\varsigma}=G_{0}$ and $U_{\varsigma}=U_{0}$.

Then, one can single out a formal power series $\hat{G}(u)=\sum_{n>0} G_{n} u^{n}$ with coefficients $G_{n}$ belonging to $\mathbb{F}$, which is the common Gevrey asymptotic expansion of order $1 / k$ relatively to $u$ on $U_{p}$ for all the maps $G_{p}$, for $0 \leq p \leq \varsigma-1$. It means that two constants $K_{p}, M_{p}>0$ can be pinpointed with the error bounds

$$
\left\|G_{p}(u)-\sum_{n=0}^{N} G_{n} u^{n}\right\|_{\mathbb{F}} \leq K_{p} M_{p}^{N+1} \Gamma\left(1+\frac{N+1}{k}\right)|u|^{N+1}
$$

for all integers $N \geq 0$, all $u \in U_{p}$, all $0 \leq p \leq \varsigma-1$.

In the next proposition, we come up with asymptotic expansions of Gevrey type for the maps $\mathbb{U}_{d_{p}, \mathfrak{o}_{q}}\left(t, u_{2}, z, \epsilon\right)$, built up in Proposition 7 , relatively to each variable $\epsilon$ and $u_{2}$.

Proposition 8 1) Let $q=q_{0} \in\left\{0, \ldots, \varsigma_{2}-1\right\}$ be a given integer. We denote $\mathbb{F}_{2, q_{0}, \beta^{\prime}, \mathcal{T}}$ the Banach space of bounded holomorphic functions on the product $\mathcal{T} \times U_{2, \mathfrak{d}_{q_{0}}} \times H_{\beta^{\prime}}$ with values in $\mathbb{C}$ equipped with the sup norm. Then, there exists a formal power series

$$
\hat{\mathbb{G}}_{1, q_{0}}(\epsilon)=\sum_{n \geq 0} G_{n, q_{0}}^{1}\left(t, u_{2}, z\right) \frac{\epsilon^{n}}{n !}
$$

with coefficients $G_{n, q_{0}}^{1}, n \geq 0$, belonging to $\mathbb{F}_{2, q_{0}, \beta^{\prime}, \mathcal{T}}$ that fulfill the next asymptotic features. For all $0 \leq p \leq \varsigma_{1}-1$, one can select two constants $K_{p}^{1}, M_{p}^{1}>0$ such that

$$
\left|\mathbb{U}_{d_{p}, \mathfrak{o}_{q_{0}}}\left(t, u_{2}, z, \epsilon\right)-\sum_{n=0}^{N} G_{n, q_{0}}^{1}\left(t, u_{2}, z\right) \frac{\epsilon^{n}}{n !}\right| \leq K_{p}^{1}\left(M_{p}^{1}\right)^{N+1} \Gamma\left(1+\frac{N+1}{k_{1}}\right)|\epsilon|^{N+1}
$$

for all integers $N \geq 0$, all $\epsilon \in \mathcal{E}_{p}$, provided that $t \in \mathcal{T}, u_{2} \in U_{2, \mathrm{~d}_{q_{0}}}$ and $z \in H_{\beta^{\prime}}$. 
2) We set $p=p_{0} \in\left\{0, \ldots, \varsigma_{1}-1\right\}$ as a prescribed integer. We denote $\mathbb{F}_{1, p_{0}, \beta^{\prime}, \mathcal{T}}$ the Banach space of bounded holomorphic functions on the product $\mathcal{T} \times H_{\beta^{\prime}} \times \mathcal{E}_{p_{0}}$ which are $\mathbb{C}$-valued, endowed with the sup norm. Then, a formal power series

$$
\hat{\mathbb{G}}_{2, p_{0}}\left(u_{2}\right)=\sum_{n \geq 0} G_{n, p_{0}}^{2}(t, z, \epsilon) \frac{u_{2}^{n}}{n !}
$$

can be singled out, whose coefficients $G_{n, p_{0}}^{2}, n \geq 0$, are located in $\mathbb{F}_{1, p_{0}, \beta^{\prime}, \mathcal{T}}$ that is subjected to the following error bounds. For all $0 \leq q \leq \varsigma_{2}-1$, two constants $K_{q}^{2}, M_{q}^{2}>0$ can be chosen with

$$
\left|\mathbb{U}_{d_{p_{0}}, \mathfrak{o}_{q}}\left(t, u_{2}, z, \epsilon\right)-\sum_{n=0}^{N} G_{n, p_{0}}^{2}(t, z, \epsilon) \frac{u_{2}^{n}}{n !}\right| \leq K_{q}^{2}\left(M_{q}^{2}\right)^{N+1} \Gamma(N+2)\left|u_{2}\right|^{N+1}
$$

for all integers $N \geq 0$, all $u_{2} \in U_{2, \mathrm{o}_{q}}$, as long as $t \in \mathcal{T}, z \in H_{\beta^{\prime}}$ and $\epsilon \in \mathcal{E}_{p_{0}}$.

Proof We discuss the first point 1$)$. For all $0 \leq p \leq \varsigma_{1}-1$, let us introduce the maps $\mathbb{G}_{1, p}$ : $\mathcal{E}_{p} \rightarrow \mathbb{F}_{2, q_{0}, \beta^{\prime}, \mathcal{T}}$ defined by

$$
\mathbb{G}_{1, p}(\epsilon):=\left(t, u_{2}, z\right) \mapsto \mathbb{U}_{d_{p}, \mathfrak{d}_{q_{0}}}\left(t, u_{2}, z, \epsilon\right) .
$$

According to the first two items of Proposition 7, we observe that for all $0 \leq p \leq \varsigma_{1}-1$,

- The maps $\mathbb{G}_{1, p}$ are bounded holomorphic on the sector $\mathcal{E}_{p}$.

- The differences $\Theta_{1, p}(\epsilon)=\mathbb{G}_{1, p+1}(\epsilon)-\mathbb{G}_{1, p}(\epsilon)$ are submitted to the bounds

$$
\|\left.\Theta_{1, p}(\epsilon)\right|_{\mathbb{F}_{2, q_{0}, \beta^{\prime}, \mathcal{T}}} \leq M_{p, 1} \exp \left(-\frac{K_{p, 1}}{|\epsilon|^{k_{1}}}\right)
$$

for the constants $M_{p, 1}, K_{p, 1}>0$ introduced in (108), whenever $\epsilon \in \mathcal{E}_{p+1} \cap \mathcal{E}_{p}$. As above, we take the convention that $\mathbb{G}_{1, \varsigma_{1}}=\mathbb{G}_{1,0}$ and $\mathcal{E}_{\varsigma_{1}}=\mathcal{E}_{0}$.

As a result, the requirements 1. and 2. of Theorem (R.S.) are fulfilled for the set of maps $\left\{\mathbb{G}_{1, p}\right\}_{0 \leq p \leq \varsigma_{1}-1}$ and we deduce the existence of the formal power series (127) which is the common Gevrey asymptotic expansion of order $1 / k_{1}$ relatively to $\epsilon$ on $\mathcal{E}_{p}$ for all the maps $\mathbb{G}_{1, p}, 0 \leq p \leq$ $\varsigma_{1}-1$. In other words, the bounds (128) hold true.

We turn our attention to the second point 2). For each $0 \leq q \leq \varsigma_{2}-1$, we set up the maps $\mathbb{G}_{2, q}: U_{2, \mathfrak{o}_{q}} \rightarrow \mathbb{F}_{1, p_{0}, \beta^{\prime}, \mathcal{T}}$ as

$$
\mathbb{G}_{2, q}\left(u_{2}\right):=(t, z, \epsilon) \mapsto \mathbb{U}_{d_{p_{0}, \mathfrak{o}_{q}}}\left(t, u_{2}, z, \epsilon\right) .
$$

According to the first and last items of Proposition 7, we notice that for each $0 \leq q \leq \varsigma_{2}-1$,

- The map $\mathbb{G}_{2, q}$ is bounded holomorphic on the sector $U_{2, \mathfrak{d}_{q}}$.

- The difference $\Theta_{2, q}\left(u_{2}\right)=\mathbb{G}_{2, q+1}\left(u_{2}\right)-\mathbb{G}_{2, q}\left(u_{2}\right)$ is exponentially flat of order 1 , with

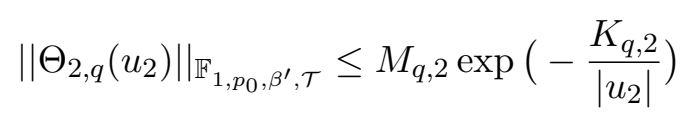

for the constants $M_{q, 2}, K_{q, 2}>0$ reached in (109), provided that $u_{2} \in U_{2, \mathfrak{o}_{q+1}} \cap U_{2, \mathfrak{o}_{q}}$. Here again, the convention that $\mathfrak{d}_{\varsigma_{2}}=\mathfrak{d}_{0}$ holds.

Thereupon, the claims 1. and 2. of Theorem (R.S.) are scored for the family of maps $\left\{\mathbb{G}_{2, q}\right\}_{0 \leq q \leq \varsigma_{2}-1}$ and the existence of the formal power series (129) which represents the common Gevrey asymptotic expansion of order 1 relatively to $u_{2}$ on $U_{2, \mathrm{~d}_{q}}$ for all the maps $\mathbb{G}_{2, q}, 0 \leq q \leq$ $\varsigma_{2}-1$ is ensured. Videlicet, the errors bounds (130) are warranted. 


\subsection{Statement of the main result}

In this subsection, we build up a family of holomorphic solutions to the main initial value problem (11) under study in this work which is set up in Subsection 2.2. These solutions possess asymptotic expansions in two different scales that turn out to be of Gevrey type.

The next statement represents the main achievement of our work.

Theorem 1 We consider sets of bounded sectors $\underline{\mathcal{U}}_{1}, \underline{\mathcal{U}}_{2}, \underline{\mathcal{E}}$ and a sector $\mathcal{T}$ that are fitting in the sense of Definition 6.

Then, under the conditions (12), (13), (14), (15), (16), (17), (18), (20) and (22) listed in Subsection 2.2, the equation

$$
\begin{aligned}
Q\left(\partial_{z}\right) u(t, z, \epsilon)=(\epsilon t)^{d_{D}}\left(t \partial_{t}\right)^{\delta_{D}} R_{D}\left(\partial_{z}\right) & u(t, z, \epsilon) \\
& +\sum_{l=1}^{D-1} \epsilon^{\Delta_{l}} t^{d_{l}}\left(t \partial_{t}\right)^{\delta_{l}} a_{l}(z, \epsilon) R_{l}\left(\partial_{z}\right) u(t, z, \epsilon)+f(t, z, \epsilon)
\end{aligned}
$$

possesses a finite set of holomorphic solutions $(t, z, \epsilon) \mapsto u_{p}(t, z, \epsilon)$, for all $p \in I_{1}$, where $I_{1}$ is the subset of $\left\{0, \ldots, \varsigma_{1}-1\right\}$ introduced in (102), on the domain $\mathcal{T} \times H_{\beta^{\prime}} \times \mathcal{E}_{p}$, provided that $\epsilon_{0}>0$ is taken close enough to 0 , for any $0<\beta^{\prime}<\beta$, for vanishing initial data $u_{p}(0, z, \epsilon) \equiv 0$.

For each $p \in I_{1}$, the solution $u_{p}$ can be expressed as Fourier inverse and double Laplace transforms

$$
\begin{aligned}
& u_{p}(t, z, \epsilon)=\mathbb{U}_{d_{p}, \pi}\left(t, \frac{1}{\log (\epsilon t)}, z, \epsilon\right) \\
& =\frac{k_{1}}{(2 \pi)^{1 / 2}} \int_{L_{d_{p, \epsilon t}}} \int_{L_{\pi, a}} \int_{-\infty}^{+\infty} \omega_{d_{p}}\left(\tau_{1}, \tau_{2}, m, \epsilon\right) \exp \left(-\left(\frac{\tau_{1}}{\epsilon t}\right)^{k_{1}}-\left(\log (\epsilon t) \tau_{2}\right)\right) e^{i z m} \frac{d \tau_{1}}{\tau_{1}} \frac{d \tau_{2}}{\tau_{2}} d m
\end{aligned}
$$

where the Borel maps $\left(\tau_{1}, \tau_{2}, m\right) \mapsto \omega_{d_{p}}\left(\tau_{1}, \tau_{2}, m, \epsilon\right)$ belong to the Banach space $F_{\left(\nu, \beta, \mu, k_{1}, \rho, a, \epsilon\right)}^{d_{p}}$ under the constraint

$$
\sup _{\epsilon \in D_{\epsilon_{0}} \backslash\{0\}}\left\|\omega_{d_{p}}\left(\tau_{1}, \tau_{2}, m, \epsilon\right)\right\|_{\left(\nu, \beta, \mu, k_{1}, \rho, a, \epsilon\right)} \leq \varpi_{p}
$$

for some well chosen constants $\varpi_{p}>0$ and radius $\rho>0$, for all $\epsilon \in D_{\epsilon_{0}} \backslash\{0\}$.

The family $\left\{u_{p}(t, z, \epsilon)\right\}_{p \in I_{1}}$ enjoys asymptotic expansions of Gevrey type in two distinguished scales of functions. Namely,

- Let us introduce the open set

$$
\mathcal{D}_{\epsilon_{0}}=\left\{\epsilon \in D_{\epsilon_{0}} \backslash\{0\} / \epsilon t \notin(-\infty, 0], \text { for all } t \in \mathcal{T}\right\} .
$$

Then, there exists a formal power series

$$
\hat{u}^{1}(t, z, \epsilon)=\sum_{n \geq 0} G_{n, q_{1}}^{1}\left(t, \frac{1}{\log (\epsilon t)}, z\right) \frac{\epsilon^{n}}{n !}
$$

whose coefficients $(t, z, \epsilon) \mapsto G_{n, q_{1}}^{1}\left(t, \frac{1}{\log (\epsilon t)}, z\right)$ are bounded holomorphic maps on $\mathcal{T} \times H_{\beta^{\prime}} \times$ $\mathcal{D}_{\epsilon_{0}}$, which represent the common asymptotic expansion of Gevrey order $1 / k_{1}$ in the scale of monomials $\left\{\epsilon^{n}\right\}_{n \geq 0}$ of the maps $u_{p}$ relatively to $\epsilon$ on every sectors $\mathcal{E}_{p}$, for all $p \in I_{1}$. 
In other words, for each $p \in I_{1}$, one can select two constants $K_{p}^{1}, M_{p}^{1}>0$ for which the next error bounds

$$
\left|u_{p}(t, z, \epsilon)-\sum_{n=0}^{N} G_{n, q_{1}}^{1}\left(t, \frac{1}{\log (\epsilon t)}, z\right) \frac{\epsilon^{n}}{n !}\right| \leq K_{p}^{1}\left(M_{p}^{1}\right)^{N+1} \Gamma\left(1+\frac{N+1}{k_{1}}\right)|\epsilon|^{N+1}
$$

hold for all integers $N \geq 0$, all $\epsilon \in \mathcal{E}_{p}$, provided that $t \in \mathcal{T}$ and $z \in H_{\beta^{\prime}}$.

- For each $p \in I_{1}$, there exists a formal series

$$
\hat{u}_{p}^{2}(t, z, \epsilon)=\sum_{n \geq 0} G_{n, p}^{2}(t, z, \epsilon) \frac{(1 / \log (\epsilon t))^{n}}{n !}
$$

with bounded holomorphic coefficients $(t, z, \epsilon) \mapsto G_{n, p}^{2}(t, z, \epsilon)$ on $\mathcal{T} \times H_{\beta^{\prime}} \times \mathcal{E}_{p}$, which stands for the asymptotic expansion of Gevrey order 1 in the scale of logarithmic functions $\left\{(1 / \log (\epsilon t))^{n}\right\}_{n \geq 0}$ of the map $u_{p}$ on the domain $\mathcal{T} \times \mathcal{E}_{p}$. It means that two constants $K_{q_{1}}^{2}, M_{q_{1}}^{2}>0$ can be pinpointed with

$$
\left|u_{p}(t, z, \epsilon)-\sum_{n=0}^{N} G_{n, p}^{2}(t, z, \epsilon) \frac{(1 / \log (\epsilon t))^{n}}{n !}\right| \leq K_{q_{1}}^{2}\left(M_{q_{1}}^{2}\right)^{N+1} \Gamma(N+2)|1 / \log (\epsilon t)|^{N+1}
$$

for all integers $N \geq 0$, all $\epsilon \in \mathcal{E}_{p}$ and $t \in \mathcal{T}$, whenever $z \in H_{\beta^{\prime}}$.

Proof According to the point 5. of Definition 6, we select the index $0 \leq q_{1} \leq \varsigma_{2}-1$ such that $\mathfrak{d}_{q_{1}}=\pi$. By definition of the principal value of the $\operatorname{logarithm} \log (z)=\ln |z|+\sqrt{-1} \arg (z)$ for $\arg (z) \in(-\pi, \pi)$, we notice that

$$
\frac{1}{\log (\epsilon t)} \in U_{2, \mathfrak{o}_{q_{1}}}=U_{2, \pi}, \lim _{t \rightarrow 0} \frac{1}{\log (\epsilon t)}=0
$$

provided that $\epsilon \in \mathcal{D}_{\epsilon_{0}}$ (given by (134)), where $\epsilon_{0}>0$ is taken small enough, for all $t \in \mathcal{T}$.

For all $p \in I_{1}$, where the set $I_{1}$ is introduced in (102), we define

$$
u_{p}(t, z, \epsilon):=\mathbb{U}_{d_{p}, \pi}\left(t, \frac{1}{\log (\epsilon t)}, z, \epsilon\right)
$$

where the map $\mathbb{U}_{d_{p}, \pi}$ is set up in Proposition 7. As a result of the definition of the set $I_{1}$ and owing to the first item of Proposition 7, we check that the map $u_{p}(t, z, \epsilon)$ represents a bounded holomorphic function on the product $\mathcal{T} \times H_{\beta^{\prime}} \times \mathcal{E}_{p}$.

According to Proposition 6, we know that for all $\epsilon \in D_{\epsilon_{0}} \backslash\{0\}$, the map $\left(u_{1}, u_{2}, z\right) \mapsto$ $U_{d_{p}, \pi}\left(u_{1}, u_{2}, z, \epsilon\right)$ represents a solution of the equation (104) which reduces to the equation (28) on the domain $U_{1, d_{p}} \times U_{2, \pi} \times H_{\beta^{\prime}}$. On the basis of the computations (27) made in Subsection 2.3, we deduce that $u_{p}(t, z, \epsilon)$ stands for a solution to the main equation (11) restated as (131) in Theorem 1 , on the domain $\mathcal{T} \times H_{\beta^{\prime}} \times \mathcal{E}_{p}$, for all $p \in I_{1}$. Moreover, since $U_{d_{p}, \pi}(0,0, z, \epsilon) \equiv 0$, we come up with the vanishing initial data $u_{p}(0, z, \epsilon) \equiv 0$. Besides, the representation (132) as Fourier Laplace transforms stems from a similar representation for the auxiliary map $U_{d_{p}, \pi}\left(u_{1}, u_{2}, z, \epsilon\right)$ in (105).

At last, the two items dealing with the asymptotic expansions properties of the maps $u_{p}$ in the two distinguished scales $\left\{\epsilon^{n}\right\}_{n \geq 0}$ and $\left\{(1 / \log (\epsilon t))^{n}\right\}_{n \geq 0}$ are direct consequences of the expansions (128) and (130) reached for the auxiliary maps $U_{d_{p}, \mathfrak{o}_{q}}\left(t, u_{2}, z, \epsilon\right)$ in Proposition 8, for $p \in I_{1}$, where the variable $u_{2}$ is merely replaced by the logarithmic function $1 / \log (\epsilon t)$ with $\epsilon \in \mathcal{E}_{p}$ and $t \in \mathcal{T}$. 


\section{Computational aspects of the formal power series asymptotic expansions}

In this section, we state that the formal power series (127) and (129), built up in Proposition 8, which represent asymptotic expansions of Gevrey type for the maps $\mathbb{U}_{d_{p}, \mathfrak{o}_{q}}\left(t, u_{2}, z, \epsilon\right)$ actually solve some linear partial differential equations. On the way, we observe that their coefficients $G_{n, q}^{1}$ and $G_{n, p}^{2}$ fulfill recursion relations that may be useful for practical purpose.

Proposition 9 Let $q=q_{0} \in\left\{0, \ldots, \varsigma_{2}-1\right\}$ be a prescribed integer. Then, the formal power series

$$
\hat{\mathbb{G}}_{1, q_{0}}(\epsilon)=\sum_{n \geq 0} G_{n, q_{0}}^{1}\left(t, u_{2}, z\right) \frac{\epsilon^{n}}{n !}
$$

with coefficients $G_{n, q_{0}}^{1}, n \geq 0$ in the space $\mathbb{F}_{2, q_{0}, \beta^{\prime}, \mathcal{T}}$, constructed in Proposition 81 ), match the next partial differential equation

$$
Q\left(\partial_{z}\right) \hat{\mathbb{G}}_{1, q_{0}}(\epsilon)=(\epsilon t)^{d_{D}} \sum_{p_{1}+p_{2}=\delta_{D}} \frac{\delta_{D} !}{p_{1} ! p_{2} !}\left(t \partial_{t}\right)^{p_{1}}(-1)^{p_{2}}\left(u_{2}^{2} \partial_{u_{2}}\right)^{p_{2}} R_{D}\left(\partial_{z}\right) \hat{\mathbb{G}}_{1, q_{0}}(\epsilon)
$$

$+\sum_{l=1}^{D-1} \epsilon^{\Delta_{l}} t^{d_{l}} \times \sum_{p_{1}+p_{2}=\delta_{l}} \frac{\delta_{l} !}{p_{1} ! p_{2} !}\left(t \partial_{t}\right)^{p_{1}}(-1)^{p_{2}}\left(u_{2}^{2} \partial_{u_{2}}\right)^{p_{2}} a_{l}(z, \epsilon) R_{l}\left(\partial_{z}\right) \hat{\mathbb{G}}_{1, q_{0}}(\epsilon)+F_{\mathfrak{d}_{q_{0}}, a}\left(\epsilon t, u_{2}, z, \epsilon\right)$.

Furthermore, the coefficients $G_{n, q_{0}}^{1}\left(t, u_{2}, z\right)$, for $n \geq 0$, are subjected to the recursion relation (145).

Proof We first display the partial differential equation that the map $\mathbb{U}_{d_{p}, \mathfrak{d}_{q_{0}}}\left(t, u_{2}, z, \epsilon\right)$ introduced in (107) turns out to fulfill. The usual chain rule allows the next computation

$$
t \partial_{t} \mathbb{U}_{d_{p}, \mathfrak{d}_{q_{0}}}\left(t, u_{2}, z, \epsilon\right)=\left(u_{1} \partial_{u_{1}} U_{d_{p}, \mathfrak{d}_{q_{0}}}\right)\left(\epsilon t, u_{2}, z, \epsilon\right)
$$

to hold for all $0 \leq p \leq \varsigma_{1}-1,0 \leq q_{0} \leq \varsigma_{2}-1$, provided that $t \in \mathcal{T}, u_{2} \in U_{2, \mathrm{o}_{0}}, z \in H_{\beta^{\prime}}$ and $\epsilon \in \mathcal{E}_{p}$. As a consequence, since the partial map $\left(u_{1}, u_{2}, z\right) \mapsto U_{d_{p}, \mathfrak{d}_{q_{0}}}\left(u_{1}, u_{2}, z, \epsilon\right)$ solves the equation (35) (where $d_{1}=d_{p}$ and $d_{2}=\mathfrak{d}_{q_{0}}$ ) on the domain $U_{1, d_{p}} \times U_{2, \mathrm{~d}_{q_{0}}} \times H_{\beta^{\prime}}$, whenever $\epsilon \in D_{\epsilon_{0}} \backslash\{0\}$, we deduce that the maps $\mathbb{U}_{d_{p}, \mathfrak{o}_{q_{0}}}\left(t, u_{2}, z, \epsilon\right)$ satisfy the next equation

$$
\begin{aligned}
& Q\left(\partial_{z}\right) \mathbb{U}_{d_{p}, \mathfrak{o}_{q_{0}}}\left(t, u_{2}, z, \epsilon\right) \\
& =(\epsilon t)^{d_{D}} \sum_{p_{1}+p_{2}=\delta_{D}} \frac{\delta_{D} !}{p_{1} ! p_{2} !}\left(t \partial_{t}\right)^{p_{1}}(-1)^{p_{2}}\left(u_{2}^{2} \partial_{u_{2}}\right)^{p_{2}} R_{D}\left(\partial_{z}\right) \mathbb{U}_{d_{p}, \mathfrak{d}_{q_{0}}}\left(t, u_{2}, z, \epsilon\right) \\
& +\sum_{l=1}^{D-1} \epsilon^{\Delta_{l}} t^{d_{l}} \times \sum_{p_{1}+p_{2}=\delta_{l}} \frac{\delta_{l} !}{p_{1} ! p_{2} !}\left(t \partial_{t}\right)^{p_{1}}(-1)^{p_{2}}\left(u_{2}^{2} \partial_{u_{2}}\right)^{p_{2}} a_{l}(z, \epsilon) R_{l}\left(\partial_{z}\right) \mathbb{U}_{d_{p}, \mathfrak{o}_{q_{0}}}\left(t, u_{2}, z, \epsilon\right) \\
& +F_{\mathfrak{d}_{q_{0}}, a}\left(\epsilon t, u_{2}, z, \epsilon\right) .
\end{aligned}
$$

as long as $t \in \mathcal{T}, u_{2} \in U_{2, \mathfrak{d}_{q_{0}}}, z \in H_{\beta^{\prime}}$ and $\epsilon \in \mathcal{E}_{p}$.

Besides, we remind the reader the next classical result relating the existence of asymptotic expansions for holomorphic maps $f$ with the continuity of their $n$-th order derivatives,

Proposition ([1], Proposition 8, p. 66) Let $f: G \mapsto \mathbb{F}$ be a holomorphic function from a bounded open sector $G$ centered at 0 into a complex Banach space $\mathbb{F}$ equipped with a norm $\|.\|_{\mathbb{F}}$. The following two statements are equivalent 
- There exists a formal power series $\hat{f}(z)=\sum_{n \geq 0} f_{n} z^{n} / n$ ! with coefficients $f_{n}$ in $\mathbb{F}$ subjected to the next feature. For all closed subsector $S$ of $G$ centered at 0 , there exists a sequence $(c(N, S))_{N \geq 0}$ of positive real numbers such that

$$
\left\|f(z)-\sum_{n=0}^{N-1} f_{n} z^{n} / n !\right\|_{\mathbb{F}} \leq c(N, S)|z|^{N}
$$

for all $z \in S$, all integers $N \geq 1$.

- All derivatives of order $n, f^{(n)}(z)$ are continuous at the origin and

$$
\lim _{z \rightarrow 0, z \in G}\left\|f^{(n)}(z)-f_{n}\right\|_{\mathbb{F}}=0
$$

for all integers $n \geq 0$.

According to the errors bounds (128), the above proposition gives rise to the next limits

$$
\lim _{\substack{\epsilon \rightarrow 0, \epsilon \in \mathcal{E}_{p}, t \in \mathcal{T}, u_{2} \in U_{2, \mathfrak{d}} \\ z \in H_{\beta^{\prime}}}}\left|\partial_{\epsilon}^{m} \mathbb{U}_{d_{p}, \mathfrak{d}_{q_{0}}}\left(t, u_{2}, z, \epsilon\right)-G_{m, q_{0}}^{1}\left(t, u_{2}, z\right)\right|=0
$$

for all integers $m \geq 0$. Now, we go back to (142) and take the derivative of order $m \geq 0$ of its left and right handside. Owing to the Leibniz rule, we get

$$
\begin{aligned}
& Q\left(\partial_{z}\right) \partial_{\epsilon}^{m} \mathbb{U}_{d_{p}, \mathfrak{d}_{q_{0}}}\left(t, u_{2}, z, \epsilon\right)=\sum_{m_{1}+m_{2}=m} \frac{m !}{m_{1} ! m_{2} !}\left(\partial_{\epsilon}^{m_{1}} \epsilon^{d_{D}}\right) t^{d_{D}} \\
& \times \sum_{p_{1}+p_{2}=\delta_{D}} \frac{\delta_{D} !}{p_{1} ! p_{2} !}\left(t \partial_{t}\right)^{p_{1}}(-1)^{p_{2}}\left(u_{2}^{2} \partial_{u_{2}}\right)^{p_{2}} R_{D}\left(\partial_{z}\right) \partial_{\epsilon}^{m_{2}} \mathbb{U}_{d_{p}, \mathfrak{d}_{q_{0}}}\left(t, u_{2}, z, \epsilon\right) \\
& \quad+\sum_{l=1}^{D-1} \sum_{m_{1}+m_{2}+m_{3}=m} \frac{m !}{m_{1} ! m_{2} ! m_{3} !}\left(\partial_{\epsilon}^{m_{1}} \epsilon^{\Delta_{l}}\right) t^{d_{l}} \\
& \quad \sum_{p_{1}+p_{2}=\delta_{l}} \frac{\delta_{l} !}{p_{1} ! p_{2} !}\left(t \partial_{t}\right)^{p_{1}}(-1)^{p_{2}}\left(u_{2}^{2} \partial_{u_{2}}\right)^{p_{2}} \partial_{\epsilon}^{m_{2}} a_{l}(z, \epsilon) R_{l}\left(\partial_{z}\right) \partial_{\epsilon}^{m_{3}} \mathbb{U}_{d_{p}, \mathfrak{o}_{q_{0}}}\left(t, u_{2}, z, \epsilon\right) \\
& +\partial_{\epsilon}^{m} F_{\mathfrak{d}_{q_{0}}, a}\left(\epsilon t, u_{2}, z, \epsilon\right)
\end{aligned}
$$

for all $m \geq 0$, all $t \in \mathcal{T}, u_{2} \in U_{2, \mathfrak{o}_{q_{0}}}, z \in H_{\beta^{\prime}}$ and $\epsilon \in \mathcal{E}_{p}$.

We let $\epsilon$ tend to 0 on the sector $\mathcal{E}_{p}$ in the equality (144). According to the limits (143) and owing that the maps $\mathbb{U}_{d_{p}, \mathfrak{d}_{q_{0}}}\left(t, u_{2}, z, \epsilon\right)$ and $G_{m, q_{0}}^{1}\left(t, u_{2}, z\right)$ are holomorphic relatively to $\left(t, u_{2}, z\right) \in \mathcal{T} \times U_{2, \mathfrak{d}_{q_{0}}} \times H_{\beta^{\prime}}$, we get the next recursion relation for the coefficients $G_{m, q_{0}}^{1}$ displayed as

$$
\begin{aligned}
& Q\left(\partial_{z}\right) G_{m, q_{0}}^{1}\left(t, u_{2}, z\right)=\sum_{m_{1}+m_{2}=m} \frac{m !}{m_{1} ! m_{2} !}\left(\partial_{\epsilon}^{m_{1}} \epsilon^{d_{D}}\right)(0) t^{d_{D}} \\
& \times \sum_{p_{1}+p_{2}=\delta_{D}} \frac{\delta_{D} !}{p_{1} ! p_{2} !}\left(t \partial_{t}\right)^{p_{1}}(-1)^{p_{2}}\left(u_{2}^{2} \partial_{u_{2}}\right)^{p_{2}} R_{D}\left(\partial_{z}\right) G_{m_{2}, q_{0}}^{1}\left(t, u_{2}, z\right) \\
& +\sum_{l=1}^{D-1} \sum_{m_{1}+m_{2}+m_{3}=m} \frac{m !}{m_{1} ! m_{2} ! m_{3} !}\left(\partial_{\epsilon}^{m_{1}} \epsilon^{\Delta_{l}}\right)(0) t^{d_{l}} \\
& \times \sum_{p_{1}+p_{2}=\delta_{l}} \frac{\delta_{l} !}{p_{1} ! p_{2} !}\left(t \partial_{t}\right)^{p_{1}}(-1)^{p_{2}}\left(u_{2}^{2} \partial_{u_{2}}\right)^{p_{2}}\left(\partial_{\epsilon}^{m_{2}} a_{l}\right)(z, 0) R_{l}\left(\partial_{z}\right) G_{m_{3}, q_{0}}^{1}\left(t, u_{2}, z\right) \\
& +\partial_{\epsilon}^{m} F_{\mathfrak{d}_{q_{0}}, a}\left(\epsilon t, u_{2}, z, \epsilon\right)_{\mid \epsilon=0}
\end{aligned}
$$


for all $m \geq 0$, provided that $t \in \mathcal{T}, u_{2} \in U_{2, \mathfrak{d}_{q_{0}}}$ and $z \in H_{\beta^{\prime}}$. It is worth noticing that the differential relation (145) represents actually a recursion for the following reason. Observe that

$$
\frac{\left(\partial_{\epsilon}^{m_{1}} \epsilon^{d_{D}}\right)(0)}{m_{1} !}=\left\{\begin{array}{ll}
0 & \text { if } m_{1} \neq d_{D} \\
1 & \text { if } m_{1}=d_{D}
\end{array}, \quad \frac{\left(\partial_{\epsilon}^{m_{1}} \epsilon^{\Delta_{l}}\right)(0)}{m_{1} !}= \begin{cases}0 & \text { if } m_{1} \neq \Delta_{l} \\
1 & \text { if } m_{1}=\Delta_{l}\end{cases}\right.
$$

for all $1 \leq l \leq D-1$. Therefore, for the summation $\sum_{m_{1}+m_{2}=m}$ inside (145), the indices $m_{2} \geq 0$ for which non vanishing terms occur satisfy $m_{2}=m-m_{1}=m-d_{D}<m$. Moreover, for the summation $\sum_{m_{1}+m_{2}+m_{3}=m}$, the indices $m_{3} \geq 0$ for which non vanishing terms take place are subjected to $m_{3}=m-m_{2}-m_{1}=m-m_{2}-\Delta_{l}<m$. As a result, through the relation (145), each term $G_{m, q_{0}}^{1}$ is expressed by means of lower terms $G_{m^{\prime}, q_{0}}^{1}$ with $m^{\prime}<m$ for all integers $m \geq 1$.

On the other hand, we know that the maps $\epsilon \mapsto \epsilon^{d_{D}}, \epsilon \mapsto \epsilon^{\Delta_{l}}, \epsilon \mapsto a_{l}(z, \epsilon)$ together with $\epsilon \mapsto F_{\mathfrak{D}_{q_{0}}, a}\left(\epsilon t, u_{2}, z, \epsilon\right)$ are analytic on the disc $D_{\epsilon_{0}}$. Their convergent Taylor expansions at 0 are expressed as follows on $D_{\epsilon_{0}}$,

$$
\begin{array}{r}
\epsilon^{d_{D}}=\sum_{m \geq 0} \frac{\left(\partial_{\epsilon}^{m} \epsilon^{d_{D}}\right)(0)}{m !} \epsilon^{m}, \epsilon^{\Delta_{l}}=\sum_{m \geq 0} \frac{\left(\partial_{\epsilon}^{m} \epsilon^{\Delta_{l}}\right)(0)}{m !} \epsilon^{m} \quad, \quad a_{l}(z, \epsilon)=\sum_{m \geq 0} \frac{\left(\partial_{\epsilon}^{m} a_{l}\right)(z, 0)}{m !} \epsilon^{m} \\
F_{\mathfrak{D}_{q_{0}}, a}\left(\epsilon t, u_{2}, z, \epsilon\right)=\sum_{m \geq 0} \frac{\partial_{\epsilon}^{m} F_{\mathfrak{d}_{q_{0}}, a}\left(\epsilon t, u_{2}, z, \epsilon\right)_{\mid \epsilon=0}}{m !} \epsilon^{m}
\end{array}
$$

from which the next plain computations are deduced:

$$
\begin{aligned}
& (\epsilon t)^{d_{D}}\left(t \partial_{t}\right)^{p_{1}}(-1)^{p_{2}}\left(u_{2}^{2} \partial_{u_{2}}\right)^{p_{2}} R_{D}\left(\partial_{z}\right) \hat{\mathbb{G}}_{1, q_{0}}(\epsilon) \\
& \quad=t^{d_{D}} \sum_{m \geq 0}\left(\sum_{m_{1}+m_{2}=m} \frac{\left(\partial_{\epsilon}^{m_{1}} \epsilon^{d_{D}}\right)(0)}{m_{1} !}\left(t \partial_{t}\right)^{p_{1}}(-1)^{p_{2}}\left(u_{2}^{2} \partial_{u_{2}}\right)^{p_{2}} R_{D}\left(\partial_{z}\right) \frac{G_{m_{2}, q_{0}}^{1}\left(t, u_{2}, z\right)}{m_{2} !}\right) \epsilon^{m}
\end{aligned}
$$

and

$$
\begin{aligned}
\epsilon^{\Delta_{l}} t^{d_{l}}\left(t \partial_{t}\right)^{p_{1}}(-1)^{p_{2}}\left(u_{2}^{2} \partial_{u_{2}}\right)^{p_{2}} a_{l}(z, \epsilon) R_{l}\left(\partial_{z}\right) \hat{\mathbb{G}}_{1, q_{0}}(\epsilon) \\
=t^{d_{l}} \sum_{m \geq 0}\left(\sum_{m_{1}+m_{2}+m_{3}=m} \frac{\left(\partial_{\epsilon}^{m_{1}} \epsilon^{\Delta_{l}}\right)(0)}{m_{1} !}\left(t \partial_{t}\right)^{p_{1}}(-1)^{p_{2}}\left(u_{2}^{2} \partial_{u_{2}}\right)^{p_{2}}\right. \\
\left.\quad \times \frac{\left(\partial_{\epsilon}^{m_{2}} a_{l}\right)(z, 0)}{m_{2} !} R_{l}\left(\partial_{z}\right) \frac{G_{m_{3}, q_{0}}^{1}\left(t, u_{2}, z\right)}{m_{3} !}\right) \epsilon^{m}
\end{aligned}
$$

At last, the recursion (145) together with the formal expansions (147), (148) prompt the forecast partial differential equation (141).

Proposition 10 Let $p=p_{0} \in\left\{0, \ldots, \varsigma_{1}-1\right\}$ be a given integer. Then, the formal power series

$$
\hat{\mathbb{G}}_{2, p_{0}}\left(u_{2}\right)=\sum_{n \geq 0} G_{n, p_{0}}^{2}(t, z, \epsilon) \frac{u_{2}^{n}}{n !}
$$

with coefficients $G_{n, p_{0}}^{2}, n \geq 0$, taken in the space $\mathbb{F}_{1, p_{0}, \beta^{\prime}, \mathcal{T}}$, arising in Proposition 8 2), is submitted to the next partial differential equation

$$
\begin{aligned}
& Q\left(\partial_{z}\right) \hat{\mathbb{G}}_{2, p_{0}}\left(u_{2}\right)=(\epsilon t)^{d_{D}} \sum_{p_{1}+p_{2}=\delta_{D}} \frac{\delta_{D} !}{p_{1} ! p_{2} !}\left(t \partial_{t}\right)^{p_{1}}(-1)^{p_{2}}\left(u_{2}^{2} \partial_{u_{2}}\right)^{p_{2}} R_{D}\left(\partial_{z}\right) \hat{\mathbb{G}}_{2, p_{0}}\left(u_{2}\right) \\
& +\sum_{l=1}^{D-1} \epsilon^{\Delta_{l}} t^{d_{l}} \times \sum_{p_{1}+p_{2}=\delta_{l}} \frac{\delta_{l} !}{p_{1} ! p_{2} !}\left(t \partial_{t}\right)^{p_{1}}(-1)^{p_{2}}\left(u_{2}^{2} \partial_{u_{2}}\right)^{p_{2}} a_{l}(z, \epsilon) R_{l}\left(\partial_{z}\right) \hat{\mathbb{G}}_{2, p_{0}}\left(u_{2}\right)+\hat{\mathbb{F}}_{2, a}\left(u_{2}\right),
\end{aligned}
$$


with forcing term $\hat{\mathbb{F}}_{2, a}\left(u_{2}\right)$ representing a formal power series displayed in (167). Moreover, the coefficients $G_{n, p_{0}}^{2}(t, z, \epsilon)$, for $n \geq 0$, fulfill the recursion relation (166).

Proof In the first instance, we state a lemma that will play an essential role in the proof.

Lemma 5 For any given integer $p_{2} \geq 1$, the expansion

$$
\left(u_{2}^{2} \partial_{u_{2}}\right)^{p_{2}}=\sum_{j=0}^{p_{2}} a_{j, p_{2}}\left(u_{2}\right) \partial_{u_{2}}^{j}
$$

holds for monomials $a_{j, p_{2}}\left(u_{2}\right), 0 \leq j \leq p_{2}$, with the shape

$$
a_{j, p_{2}}\left(u_{2}\right)=\beta_{j, p_{2}} u_{2}^{p_{2}+j}
$$

for suitable integers $\beta_{j, p_{2}} \geq 0$ with the constraints $\beta_{0, p_{2}}=0, \beta_{p_{2}, p_{2}}=1$, for all $p_{2} \geq 1$.

Proof The existence of polynomials $a_{j, p_{2}}\left(u_{2}\right)$ warranting the equality (150) is obtained by induction. On the way, it can be shown that the set $a_{j, p_{2}}\left(u_{2}\right), 0 \leq j \leq p_{2}$ is asked to fulfill the next recursions

$$
\begin{aligned}
& a_{j, p_{2}+1}\left(u_{2}\right)=u_{2}^{2}\left(\partial_{u_{2}} a_{j, p_{2}}\left(u_{2}\right)\right)+u_{2}^{2} a_{j-1, p_{2}}\left(u_{2}\right), a_{0, p_{2}+1}\left(u_{2}\right)=u_{2}^{2} \partial_{u_{2}} a_{0, p_{2}}\left(u_{2}\right), \\
& a_{p_{2}+1, p_{2}+1}\left(u_{2}\right)=u_{2}^{2} a_{p_{2}, p_{2}}\left(u_{2}\right)
\end{aligned}
$$

for all integers $p_{2} \geq 1,1 \leq j \leq p_{2}$.

Since $a_{0,1}\left(u_{2}\right)=0$, we deduce that $a_{0, p_{2}}\left(u_{2}\right) \equiv 0$, for all $p_{2} \geq 1$ and observing that $a_{1,1}\left(u_{2}\right)=$ $u_{2}^{2}$, we come up with $a_{p_{2}, p_{2}}\left(u_{2}\right)=u_{2}^{2 p_{2}}$, for all $p_{2} \geq 1$. Furthermore, by plugging the expression (151) into the relations (152), we get that the integers $\beta_{j, p_{2}}$ are queried to satisfy the next recursion

$$
\beta_{j, p_{2}+1}=\beta_{j, p_{2}}\left(p_{2}+j\right)+\beta_{j-1, p_{2}}, \quad \beta_{0, p_{2}}=0, \quad \beta_{p_{2}, p_{2}}=1
$$

for all integers $p_{2} \geq 1,1 \leq j \leq p_{2}$. The result follows.

In order to be able to handle higher order derivatives relatively to $u_{2}$ of the maps $\mathbb{U}_{d_{p_{0}}, \mathfrak{o}_{q}}$, for some fixed $0 \leq q \leq \varsigma_{2}-1$, we remodel the equation (142) by means of the above lemma in the next form

$$
\begin{aligned}
& Q\left(\partial_{z}\right) \mathbb{U}_{d_{p_{0}, \mathfrak{o}_{q}}}\left(t, u_{2}, z, \epsilon\right) \\
& =(\epsilon t)^{d_{D}} \sum_{\substack{p_{1}+p_{2}=\delta_{D} \\
p_{2} \geq 1}} \frac{\delta_{D} !}{p_{1} ! p_{2} !}\left(t \partial_{t}\right)^{p_{1}}(-1)^{p_{2}} \times \sum_{j=1}^{p_{2}} a_{j, p_{2}}\left(u_{2}\right) \partial_{u_{2}}^{j} R_{D}\left(\partial_{z}\right) \mathbb{U}_{d_{p_{0}}, \mathfrak{o}_{q}}\left(t, u_{2}, z, \epsilon\right) \\
& \quad+(\epsilon t)^{d_{D}}\left(t \partial_{t}\right)^{\delta_{D}} R_{D}\left(\partial_{z}\right) \mathbb{U}_{d_{p_{0}}, \mathfrak{o}_{q}}\left(t, u_{2}, z, \epsilon\right) \\
& +\sum_{l=1}^{D-1} \epsilon^{\Delta_{l}} t^{d_{l}} \times \sum_{\substack{p_{1}+p_{2}=\delta_{l} \\
p_{2} \geq 1}} \frac{\delta_{l} !}{p_{1} ! p_{2} !}\left(t \partial_{t}\right)^{p_{1}}(-1)^{p_{2}} \times \sum_{j=1}^{p_{2}} a_{j, p_{2}}\left(u_{2}\right) \partial_{u_{2}}^{j} a_{l}(z, \epsilon) R_{l}\left(\partial_{z}\right) \mathbb{U}_{d_{p_{0}}, \mathfrak{o}_{q}}\left(t, u_{2}, z, \epsilon\right) \\
& +\sum_{l=1}^{D-1} \epsilon^{\Delta_{l}} t^{d_{l}}\left(t \partial_{t}\right)^{\delta_{l}} a_{l}(z, \epsilon) R_{l}\left(\partial_{z}\right) \mathbb{U}_{d_{p_{0}}, \mathfrak{o}_{q}}\left(t, u_{2}, z, \epsilon\right)+F_{\mathfrak{d}_{q}, a}\left(\epsilon t, u_{2}, z, \epsilon\right)
\end{aligned}
$$


as long as $t \in \mathcal{T}, u_{2} \in U_{2, \mathbb{o}_{q}}, z \in H_{\beta^{\prime}}$ and $\epsilon \in \mathcal{E}_{p_{0}}$. Besides, by dint of Proposition ([1], Proposition 8, p.66) stated in the proof of Proposition 9 and according to the error bounds (130), we deduce the next uniform limits

$$
\lim _{\substack{u_{2} \rightarrow 0, u_{2} \in U_{2, \mathrm{o}_{q}}}} \sup _{\substack{t \in \mathcal{T}, \epsilon \in \mathcal{E}_{p_{0}} \\ z \in H_{\beta^{\prime}}}}\left|\partial_{u_{2}}^{m} \mathbb{U}_{d_{p_{0}}, \mathfrak{d}_{q}}\left(t, u_{2}, z, \epsilon\right)-G_{m, p_{0}}^{2}(t, z, \epsilon)\right|=0
$$

for all integers $m \geq 0$.

We take the derivative of order $m \geq 0$ relatively to $u_{2}$ on the left and right handside of (153). With the help of the Leibniz rule, we arrive at

$$
\begin{aligned}
& Q\left(\partial_{z}\right) \partial_{u_{2}}^{m} \mathbb{U}_{d_{p_{0}, \mathfrak{o}_{q}}}\left(t, u_{2}, z, \epsilon\right) \\
& =(\epsilon t)^{d_{D}} \sum_{\substack{p_{1}+p_{2}=\delta_{D} \\
p_{2} \geq 1}} \frac{\delta_{D} !}{p_{1} ! p_{2} !}\left(t \partial_{t}\right)^{p_{1}}(-1)^{p_{2}} \times \sum_{j=1}^{p_{2}} \sum_{m_{1}+m_{2}=m} \frac{m !}{m_{1} ! m_{2} !} \partial_{u_{2}}^{m_{1}} a_{j, p_{2}}\left(u_{2}\right) \\
& \times \partial_{u_{2}}^{m_{2}+j} R_{D}\left(\partial_{z}\right) \mathbb{U}_{d_{p_{0}}, \mathfrak{o}_{q}}\left(t, u_{2}, z, \epsilon\right)+(\epsilon t)^{d_{D}}\left(t \partial_{t}\right)^{\delta_{D}} R_{D}\left(\partial_{z}\right) \partial_{u_{2}}^{m} \mathbb{U}_{d_{p_{0}}, \mathfrak{o}_{q}}\left(t, u_{2}, z, \epsilon\right) \\
& +\sum_{l=1}^{D-1} \epsilon^{\Delta_{l}} t^{d_{l}} \times \sum_{\substack{p_{1}+p_{2}=\delta_{l} \\
p_{2} \geq 1}} \frac{\delta_{l} !}{p_{1} ! p_{2} !}\left(t \partial_{t}\right)^{p_{1}}(-1)^{p_{2}} \times \sum_{j=1}^{p_{2}} \sum_{m_{1}+m_{2}=m} \frac{m !}{m_{1} ! m_{2} !} \partial_{u_{2}}^{m_{1}} a_{j, p_{2}}\left(u_{2}\right) \\
& \times a_{l}(z, \epsilon) R_{l}\left(\partial_{z}\right) \partial_{u_{2}}^{m_{2}+j} \mathbb{U}_{d_{p_{0}}, \mathfrak{o}_{q}}\left(t, u_{2}, z, \epsilon\right)+\sum_{l=1}^{D-1} \epsilon^{\Delta_{l}} t^{d_{l}}\left(t \partial_{t}\right)^{\delta_{l}} a_{l}(z, \epsilon) R_{l}\left(\partial_{z}\right) \partial_{u_{2}}^{m_{2}} \mathbb{U}_{d_{p_{0}, \mathfrak{o}_{q}}\left(t, u_{2}, z, \epsilon\right)} \\
& +\partial_{u_{2}}^{m} F_{\mathfrak{o}_{q}, a}\left(\epsilon t, u_{2}, z, \epsilon\right)
\end{aligned}
$$

for all $m \geq 0$, provided that $t \in \mathcal{T}, u_{2} \in U_{2, \mathfrak{d}_{q}}, z \in H_{\beta^{\prime}}$ and $\epsilon \in \mathcal{E}_{p_{0}}$.

In order to allow the variable $u_{2}$ become close to the origin on the sector $U_{2, \mathfrak{o}_{q}}$ in the above relation, the next lemma is needed

Lemma 6 There exists a sequence $\left\{F_{2, a, m}(t, z, \epsilon)\right\}_{m \geq 0}$ of bounded holomorphic maps on the product $\mathcal{T} \times H_{\beta^{\prime}} \times \mathcal{E}_{p_{0}}$ such that

$$
\lim _{\substack{u_{2} \rightarrow 0, u_{2} \in U_{2, \mathfrak{o}_{q}}}} \sup _{\substack{t \in \mathcal{T}, \epsilon \in \mathcal{E}_{p_{0}} \\ z \in H_{\beta^{\prime}}}}\left|\partial_{u_{2}}^{m} F_{\mathfrak{d}_{q}, a}\left(\epsilon t, u_{2}, z, \epsilon\right)-F_{2, a, m}(t, z, \epsilon)\right|
$$

for all integers $m \geq 0$.

Proof From the expansion (19) with coefficients $\mathcal{F}_{j_{1}, j_{2}}(m, \epsilon)$ subjected to (18), we can recast the double Laplace and inverse Fourier representation $(29)$ of $F_{\mathfrak{D}_{q}, a}\left(\epsilon t, u_{2}, z, \epsilon\right)$ as a sum

$$
F_{\mathfrak{d}_{q}, a}\left(\epsilon t, u_{2}, z, \epsilon\right)=\sum_{j_{1} \in J_{1}} F_{\mathfrak{d}_{q}, a, j_{1}}\left(u_{2}, z, \epsilon\right) \Gamma\left(\frac{j_{1}}{k_{1}}\right)(\epsilon t)^{j_{1}}
$$

where

$$
F_{\mathfrak{D}_{q}, a, j_{1}}\left(u_{2}, z, \epsilon\right)=\sum_{j_{2} \in J_{2}} \mathbb{F}_{j_{1}, j_{2}}(z, \epsilon) \int_{L_{\mathfrak{d}_{q}, a}} \tau_{2}^{j_{2}} \exp \left(-\frac{\tau_{2}}{u_{2}}\right) \frac{d \tau_{2}}{\tau_{2}}
$$

with

$$
\mathbb{F}_{j_{1}, j_{2}}(z, \epsilon)=\frac{1}{(2 \pi)^{1 / 2}} \int_{-\infty}^{+\infty} \mathcal{F}_{j_{1}, j_{2}}(m, \epsilon) e^{i z m} d m
$$


for all integers $j_{1} \in J_{1}, j_{2} \in J_{2}$, where $t \in \mathcal{T}, u_{2} \in U_{2, \mathrm{o}_{q}}, z \in H_{\beta^{\prime}}$ and $\epsilon \in \mathcal{E}_{p_{0}}$. Using the parametrization $\tau_{2}=\rho_{2} \exp \left(\sqrt{-1} \mathfrak{d}_{q}\right)$ for $0 \leq \rho_{2} \leq a$, we can rewrite

$$
\int_{L_{\mathfrak{o}_{q}, a}} \tau_{2}^{j_{2}-1} \exp \left(-\frac{\tau_{2}}{u_{2}}\right) d \tau_{2}=\left(\exp \left(\sqrt{-1} \mathfrak{d}_{q}\right)\right)^{j_{2}} \int_{0}^{a} \rho_{2}^{j_{2}-1} \exp \left(-\rho_{2} \frac{\exp \left(\sqrt{-1} \mathfrak{d}_{q}\right)}{u_{2}}\right) d \rho_{2}
$$

At this stage, we observe that this last integral can be explicitely computed. Indeed, the next recursion relation

$$
I_{m, 1 / A}=-A e^{-a / A} a^{m}+A m I_{m-1,1 / A} \quad, \quad I_{0,1 / A}=A\left(1-e^{-a / A}\right)
$$

holds for the truncated Laplace integral $I_{m, 1 / A}=\int_{0}^{a} x^{m} e^{-x / A} d x$, for any given positive real number $a>0$, non vanishing complex number $A \in \mathbb{C}^{*}$ and all integers $m \geq 1$. We deduce the existence of polynomials $\mathbb{P}_{a, j_{2}-1}^{1}(X)$ and $\mathbb{P}_{a, j_{2}-1}^{2}(X)$ with real coefficients relying on $a, j_{2}$ such that

$$
\begin{aligned}
& F_{\mathfrak{d}_{q}, a, j_{1}}\left(u_{2}, z, \epsilon\right)=\sum_{j_{2} \in J_{2}} \mathbb{F}_{j_{1}, j_{2}}(z, \epsilon)\left(\exp \left(\sqrt{-1} \mathfrak{d}_{q}\right)\right)^{j_{2}} \\
& \quad \times\left(\mathbb{P}_{a, j_{2}-1}^{2}\left(u_{2} \exp \left(-\sqrt{-1} \mathfrak{d}_{q}\right)\right)+\mathbb{P}_{a, j_{2}-1}^{1}\left(u_{2} \exp \left(-\sqrt{-1} \mathfrak{d}_{q}\right)\right) \exp \left(-a \frac{\exp \left(\sqrt{-1} \mathfrak{d}_{q}\right)}{u_{2}}\right)\right)
\end{aligned}
$$

provided that $u_{2} \in U_{2, \mathfrak{d}_{q}}, z \in H_{\beta^{\prime}}$ and $\epsilon \in \mathcal{E}_{p_{0}}$.

For this reason, for any prescribed integer $m \geq 0$, all $j_{2} \in J_{2}$ and given $a>0$, a polynomial $\mathbb{P}_{a, j_{2}-1, m}^{2}(X) \in \mathbb{R}[X]$ and a rational function $\mathbb{Q}_{a, j_{2}-1, m}^{1}(X) \in \mathbb{R}(X)$ with one single pole at $X=0$ can be singled out such that

$$
\begin{aligned}
& \partial_{u_{2}}^{m} F_{\mathfrak{d}_{q}, a, j_{1}}\left(u_{2}, z, \epsilon\right)=\sum_{j_{2} \in J_{2}} \mathbb{F}_{j_{1}, j_{2}}(z, \epsilon)\left(\exp \left(\sqrt{-1} \mathfrak{d}_{q}\right)\right)^{j_{2}} \\
& \times\left(\mathbb{P}_{a, j_{2}-1, m}^{2}\left(u_{2} \exp \left(-\sqrt{-1} \mathfrak{d}_{q}\right)\right)+\mathbb{Q}_{a, j_{2}-1, m}^{1}\left(u_{2} \exp \left(-\sqrt{-1} \mathfrak{d}_{q}\right)\right) \exp \left(-a \frac{\exp \left(\sqrt{-1} \mathfrak{d}_{q}\right)}{u_{2}}\right)\right)
\end{aligned}
$$

whenever $u_{2} \in U_{2, \mathfrak{o}_{q}}, z \in H_{\beta^{\prime}}$ and $\epsilon \in \mathcal{E}_{p_{0}}$.

In the sequel, we set

$$
F_{2, a, m}(t, z, \epsilon)=\sum_{j_{1} \in J_{1}}\left(\sum_{j_{2} \in J_{2}} \mathbb{F}_{j_{1}, j_{2}}(z, \epsilon)\left(\exp \left(\sqrt{-1} \mathfrak{d}_{q}\right)\right)^{j_{2}} \mathbb{P}_{a, j_{2}-1, m}^{2}(0)\right) \Gamma\left(\frac{j_{1}}{k_{1}}\right)(\epsilon t)^{j_{1}}
$$

which represents a bounded holomorphic map on the product $\mathcal{T} \times H_{\beta^{\prime}} \times \mathcal{E}_{p_{0}}$, for all integers $m \geq 0$. As a result, the next bounds

$$
\begin{aligned}
\sup _{\substack{t \in \mathcal{T}, \epsilon \in \mathcal{E}_{p_{0}} \\
z \in H_{\beta^{\prime}}}}\left|\partial_{u_{2}}^{m} F_{\mathfrak{d}_{q}, a}\left(\epsilon t, u_{2}, z, \epsilon\right)-F_{2, a, m}(t, z, \epsilon)\right| \leq \sum_{j_{1} \in J_{1}}\left(\sum_{j_{2} \in J_{2}} \sup _{\substack{z \in H_{\beta^{\prime}} \\
\epsilon \in \mathcal{E}_{p_{0}}}}\left|\mathbb{F}_{j_{1}, j_{2}}(z, \epsilon)\right|\right. \\
\times\left[\left|\mathbb{P}_{a, j_{2}-1, m}^{2}\left(u_{2} \exp \left(-\sqrt{-1} \mathfrak{d}_{q}\right)\right)-\mathbb{P}_{a, j_{2}-1, m}^{2}(0)\right|\right. \\
\left.\left.+\left|\mathbb{Q}_{a, j_{2}-1, m}^{1}\left(u_{2} \exp \left(-\sqrt{-1} \mathfrak{d}_{q}\right)\right) \exp \left(-a \frac{\exp \left(\sqrt{-1} \mathfrak{d}_{q}\right)}{u_{2}}\right)\right|\right]\right) \Gamma\left(\frac{j_{1}}{k_{1}}\right)\left(\epsilon_{0} r_{\mathcal{T}}\right)^{j_{1}}
\end{aligned}
$$

hold for all $u_{2} \in U_{2, \mathfrak{o}_{q}}$. By continuity at the origin, we notice that

$$
\lim _{\substack{u_{2} \rightarrow 0 \\ u_{2} \in U_{2, \mathrm{o}_{q}}}}\left|\mathbb{P}_{a, j_{2}-1, m}^{2}\left(u_{2} \exp \left(-\sqrt{-1} \mathfrak{d}_{q}\right)\right)-\mathbb{P}_{a, j_{2}-1, m}^{2}(0)\right|=0
$$


and according to the growth rate comparison between polynomials and exponential functions, we check that

$$
\lim _{\substack{u_{2} \rightarrow 0 \\ u_{2} \in U_{2, \mathfrak{o}_{q}}}}\left|\mathbb{Q}_{a, j_{2}-1, m}^{1}\left(u_{2} \exp \left(-\sqrt{-1} \mathfrak{d}_{q}\right)\right) \exp \left(-a \frac{\exp \left(\sqrt{-1} \mathfrak{d}_{q}\right)}{u_{2}}\right)\right|=0
$$

since (103) holds provided that $u_{2} \in U_{2, \mathrm{o}_{q}}$. The lemma 6 follows at last from the gathering of (163), (164) and (165).

We let $u_{2}$ tend to 0 on the sector $U_{2, \mathfrak{o}_{q}}$ in the equality (155). Keeping in mind the uniform limits (154) along with (156) and the fact that both maps $\mathbb{U}_{d_{p_{0}}, \mathfrak{o}_{q}}\left(t, u_{2}, z, \epsilon\right)$ and $G_{m, p_{0}}^{2}(t, z, \epsilon)$ are holomorphic w.r.t $(t, z) \in \mathcal{T} \times H_{\beta^{\prime}}$, we arrive at the next recursion relation for the coefficients $G_{m, p_{0}}^{2}$ written in the form

$$
\begin{aligned}
& Q\left(\partial_{z}\right) G_{m, p_{0}}^{2}(t, z, \epsilon)-(\epsilon t)^{d_{D}}\left(t \partial_{t}\right)^{\delta_{D}} R_{D}\left(\partial_{z}\right) G_{m, p_{0}}^{2}(t, z, \epsilon) \\
& -\sum_{l=1}^{D-1} \epsilon^{\Delta_{l}} t^{d_{l}}\left(t \partial_{t}\right)^{\delta_{l}} a_{l}(z, \epsilon) R_{l}\left(\partial_{z}\right) G_{m, p_{0}}^{2}(t, z, \epsilon)=(\epsilon t)^{d_{D}} \sum_{\substack{p_{1}+p_{2}=\delta_{D} \\
p_{2} \geq 1}} \frac{\delta_{D} !}{p_{1} ! p_{2} !}\left(t \partial_{t}\right)^{p_{1}}(-1)^{p_{2}} \\
& \quad \times \sum_{j=1}^{p_{2}} \sum_{m_{1}+m_{2}=m} \frac{m !}{m_{1} ! m_{2} !}\left(\partial_{u_{2}}^{m_{1}} a_{j, p_{2}}\right)(0) R_{D}\left(\partial_{z}\right) G_{m_{2}+j, p_{0}}^{2}(t, z, \epsilon) \\
& +\sum_{l=1}^{D-1} \epsilon^{\Delta_{l}} t^{d_{l}} \times \sum_{\substack{p_{1}+p_{2}=\delta_{l} \\
p_{2} \geq 1}} \frac{\delta_{l} !}{p_{1} ! p_{2} !}\left(t \partial_{t}\right)^{p_{1}}(-1)^{p_{2}} \times \sum_{j=1}^{p_{2}} \sum_{m_{1}+m_{2}=m} \frac{m !}{m_{1} ! m_{2} !}\left(\partial_{u_{2}}^{m_{1}} a_{j, p_{2}}\right)(0) \\
& \times a_{l}(z, \epsilon) R_{l}\left(\partial_{z}\right) G_{m_{2}+j, p_{0}}^{2}(t, z, \epsilon)+F_{2, a, m}(t, z, \epsilon)
\end{aligned}
$$

for all $m \geq 0$, whenever $t \in \mathcal{T}, z \in H_{\beta^{\prime}}$ and $\epsilon \in \mathcal{E}_{p_{0}}$.

We need to explain the reason for which the relation (166) turns out to be a recursion. Indeed, according to Lemma 5, we remark that

$$
\frac{\left(\partial_{u_{2}}^{m_{1}} a_{j, p_{2}}\right)(0)}{m_{1} !}=\beta_{j, p_{2}} \frac{\left(\partial_{u_{2}}^{m_{1}} u_{2}^{p_{2}+j}\right)(0)}{m_{1} !}= \begin{cases}0 & \text { if } m_{1} \neq p_{2}+j \\ \beta_{j, p_{2}} & \text { if } m_{1}=p_{2}+j\end{cases}
$$

for all integers $p_{2} \geq 1$ and $1 \leq j \leq p_{2}$. Hence, inside the summation $\sum_{m_{1}+m_{2}=m}$ blocks from (166), the indices $m_{2} \geq 0$ for which non vanishing terms appear are required to satisfy $m_{2}+j=m-m_{1}+j=m-p_{2}<m$ since $p_{2} \geq 1$. Consequently, by dint of the relation (166), each term $G_{m, p_{0}}^{2}$ can be expressed through lower terms $G_{m^{\prime}, p_{0}}^{2}$, with $m^{\prime}<m$ for all integers $m \geq 1$.

Besides, we set up the next formal series

$$
\hat{\mathbb{F}}_{2, a}\left(u_{2}\right)=\sum_{m \geq 0} F_{2, a, m}(t, z, \epsilon) \frac{u_{2}^{m}}{m !}
$$

with coefficients $F_{2, a, m}(t, z, \epsilon)$ that are built up in Lemma 6 and belong to the Banach space $\mathbb{F}_{1, p_{0}, \beta^{\prime}, \mathcal{T}}$ (defined in Proposition 82 )). For all integers $p_{2} \geq 1$ and $1 \leq j \leq p_{2}$, the monomials $u_{2} \mapsto a_{j, p_{2}}\left(u_{2}\right)$ are analytic on $\mathbb{C}$ and their Taylor expansions are expressed through

$$
a_{j, p_{2}}\left(u_{2}\right)=\sum_{m \geq 0} \frac{\left(\partial_{u_{2}}^{m} a_{j, p_{2}}\right)(0)}{m !} u_{2}^{m}
$$


according to which the next trifling computation

$$
a_{j, p_{2}}\left(u_{2}\right) \partial_{u_{2}}^{j} \hat{\mathbb{G}}_{2, p_{0}}\left(u_{2}\right)=\sum_{m \geq 0}\left(\sum_{m_{1}+m_{2}=m} \frac{\left(\partial_{u_{2}}^{m_{1}} a_{j, p_{2}}\right)(0)}{m_{1} !} \frac{G_{m_{2}+j}^{2}(t, z, \epsilon)}{m_{2} !}\right) u_{2}^{m}
$$

can be obtained. Hence, on account of the recursion (166) along with the formal expansions (169), we come up with the next partial differential equation solved by the formal series $\hat{\mathbb{G}}_{2, p_{0}}\left(u_{2}\right)$,

$$
\begin{aligned}
& Q\left(\partial_{z}\right) \hat{\mathbb{G}}_{2, p_{0}}\left(u_{2}\right)=(\epsilon t)^{d_{D}} \times \sum_{\substack{p_{1}+p_{2}=\delta_{D} \\
p_{2} \geq 1}} \frac{\delta_{D} !}{p_{1} ! p_{2} !}\left(t \partial_{t}\right)^{p_{1}}(-1)^{p_{2}} \times \sum_{j=1}^{p_{2}} a_{j, p_{2}}\left(u_{2}\right) \partial_{u_{2}}^{j} R_{D}\left(\partial_{z}\right) \hat{\mathbb{G}}_{2, p_{0}}\left(u_{2}\right) \\
& +(\epsilon t)^{d_{D}}\left(t \partial_{t}\right)^{\delta_{D}} R_{D}\left(\partial_{z}\right) \hat{\mathbb{G}}_{2, p_{0}}\left(u_{2}\right) \\
& +\sum_{l=1}^{D-1} \epsilon^{\Delta_{l}} t^{d_{l}} \times \sum_{\substack{p_{1}+p_{2}=\delta_{l} \\
p_{2} \geq 1}} \frac{\delta_{l} !}{p_{1} ! p_{2} !}\left(t \partial_{t}\right)^{p_{1}}(-1)^{p_{2}} \times \sum_{j=1}^{p_{2}} a_{j, p_{2}}\left(u_{2}\right) \partial_{u_{2}}^{j} a_{l}(z, \epsilon) R_{l}\left(\partial_{z}\right) \hat{\mathbb{G}}_{2, p_{0}}\left(u_{2}\right) \\
& +\sum_{l=1}^{D-1} \epsilon^{\Delta_{l}} t^{d_{l}}\left(t \partial_{t}\right)^{\delta_{l}} a_{l}(z, \epsilon) R_{l}\left(\partial_{z}\right) \hat{\mathbb{G}}_{2, p_{0}}\left(u_{2}\right)+\hat{\mathbb{F}}_{2, a}\left(u_{2}\right)
\end{aligned}
$$

which is tantamount to the foreseen equation (149) by means of Lemma 5.

\section{References}

[1] W. Balser, Formal power series and linear systems of meromorphic ordinary differential equations. Universitext. Springer-Verlag, New York, 2000. xviii+299 pp.

[2] M. S. Baouendi, C. Goulaouic, Cauchy problems with characteristic initial hypersurface. Comm. Pure Appl. Math. 26 (1973), 455-475.

[3] G. Chen, A. Lastra, S. Malek, Parametric Gevrey asymptotics in two complex time variables through truncated Laplace transforms. Adv. Difference Equ. 2020, Paper No. 307, 31 pp.

[4] O. Costin, S. Tanveer, Existence and uniqueness for a class of nonlinear higher-order partial differential equations in the complex plane. Comm. Pure Appl. Math. 53 (2000), no. 9, 10921117.

[5] O. Costin, S. Tanveer, Short time existence and Borel summability in the Navier-Stokes equation in $\mathbb{R}^{3}$, Comm. Partial Differential Equations 34 (2009), no. 7-9, 785-817.

[6] A. Erdélyi, Asymptotic expansions. Dover Publications, Inc., New York, 1956. vi+108 pp.

[7] R. Gérard, H. Tahara, Singular nonlinear partial differential equations. Aspects of Mathematics. Friedr. Vieweg and Sohn, Braunschweig, 1996. viii+269 pp.

[8] P. Hsieh, Y. Sibuya, Basic theory of ordinary differential equations. Universitext. SpringerVerlag, New York, 1999.

[9] A. Lastra, S. Malek, On parametric Gevrey asymptotics for some nonlinear initial value Cauchy problems. J. Differential Equations 259 (2015), no. 10, 5220-5270. 
[10] A. Lastra, S. Malek, On parametric Gevrey asymptotics for initial value problems with infinite order irregular singularity and linear fractional transforms. Adv. Difference Equ. 2018, Paper No. 386, 40 p. (2018).

[11] A. Lastra, S. Malek, On parametric Gevrey asymptotics for some initial value problems in two asymmetric complex time variables, Result. Math. 73, No. 4, Paper No. 155, 46p. (2018).

[12] A. Lastra, S. Malek, On parametric Gevrey asymptotics for some nonlinear initial value problems in symmetric complex time variables, Asymptotic Anal. 118, No. 1-2, 49-79 (2020).

[13] A. Lastra, S. Malek, On singularly perturbed linear initial value problems with mixed irregular and Fuchsian time singularities. J. Geom. Anal. 30, No. 4, 3872-3922 (2020).

[14] S. Malek, On Gevrey asymptotics for some nonlinear integro-differential equations. J. Dyn. Control Syst. 16 (2010), no. 3, 377-406.

[15] T. Mandai, Existence and non-existence of null-solutions for some non-Fuchsian partial differential operators with T-dependent coefficients. Nagoya Math. J. 122, 115-137 (1991).

[16] T. Mandai, The method of Frobenius to Fuchsian partial differential equations. J. Math. Soc. Japan 52, No. 3, 645-672 (2000).

[17] P. Mardesic, M. Resman, J.-P. Rolin, V. Zupanovic, Normal forms and embeddings for power-log transseries. Adv. Math. 303, 888-953 (2016).

[18] F. Olver, Asymptotics and special functions. Computer Science and Applied Mathematics. Academic Press, New York-London, 1974. xvi+572 pp.

[19] H. Tahara, Fuchsian type equations and Fuchsian hyperbolic equations. Jap. J. Math., New Ser. 5, 245-347 (1979).

[20] H. Tahara, Singular hyperbolic systems. V: Asymptotic expansions for Fuchsian hyperbolic partial differential equations. J. Math. Soc. Japan 36, 449-473 (1984).

[21] H. Tahara, On the singular solutions of nonlinear singular partial differential equations. I. J. Math. Soc. Japan 53 (2001), no. 3, 711-729.

[22] H. Tahara, H. Yamazawa, Multisummability of formal solutions to the Cauchy problem for some linear partial differential equations, Journal of Differential equations, Volume 255, Issue 10, 15 November 2013, pages 3592-3637.

[23] H. Yamazawa, On multisummability of formal solutions with logarithm terms for some linear partial differential equations. Funkcial. Ekvac. 60 (2017), no. 3, 371-406. 